\title{
Two-loop planar master integrals for Higgs $\rightarrow 3$ partons with full heavy-quark mass dependence
}

\author{
Roberto Bonciani, ${ }^{a, b}$ Vittorio Del Duca, ${ }^{c, d}$ Hjalte Frellesvig, ${ }^{e}$ Johannes M. Henn, ${ }^{f}$ \\ Francesco Moriello ${ }^{a, b, c}$ and Vladimir A. Smirnov ${ }^{g}$ \\ ${ }^{a}$ Dipartimento di Fisica, Sapienza - Università di Roma, \\ Piazzale Aldo Moro 5, 00185, Rome, Italy \\ ${ }^{b}$ INFN Sezione di Roma, Piazzale Aldo Moro 2, 00185, Rome, Italy \\ ${ }^{c}$ ETH Zurich, Institut fur theoretische Physik, Wolfgang-Paulistr. 27, 8093, Zurich, Switzerland \\ ${ }^{d}$ INFN Laboratori Nazionali di Frascati, 00044 Frascati, Roma, Italy \\ ${ }^{e}$ Institute of Nuclear and Particle Physics, NCSR Demokritos, Agia Paraskevi, 15310, Greece \\ ${ }^{f}$ PRISMA Cluster of Excellence, Johannes Gutenberg University, 55099 Mainz, Germany \\ ${ }^{g}$ Skobeltsyn Inst. of Nuclear Physics of Moscow State University, 119991 Moscow, Russia \\ E-mail: roberto.bonciani@roma1.infn.it, delducav@itp.phys.ethz.ch, \\ frellesvig@inp.demokritos.gr, henn@uni-mainz.de, \\ fmoriell@phys.ethz.ch, smirnov@theory.sinp.msu.ru
}

ABSTRACT: We present the analytic computation of all the planar master integrals which contribute to the two-loop scattering amplitudes for Higgs $\rightarrow 3$ partons, with full heavyquark mass dependence. These are relevant for the NNLO corrections to fully inclusive Higgs production and to the NLO corrections to Higgs production in association with a jet, in the full theory. The computation is performed using the differential equations method. Whenever possible, a basis of master integrals that are pure functions of uniform weight is used. The result is expressed in terms of one-fold integrals of polylogarithms and elementary functions up to transcendental weight four. Two integral sectors are expressed in terms of elliptic integrals. We show that by introducing a one-dimensional parametrization of the integrals the relevant second order differential equation can be readily solved, and the solution can be expressed to all orders of the dimensional regularization parameter in terms of iterated integrals over elliptic kernels. We express the result for the elliptic sectors in terms of two and three-fold iterated integrals, which we find suitable for numerical evaluations. This is the first time that four-point multiscale Feynman integrals have been computed in a fully analytic way in terms of elliptic integrals.

KeYwords: Perturbative QCD, Scattering Amplitudes

ARXIV EPRINT: 1609.06685 


\section{Contents}

1 Introduction 1

2 Notations and conventions $\quad 4$

3 Differential equations $\quad 7$

$\begin{array}{lll}3.1 & \text { General features of differential equations for Feynman integrals } & 7\end{array}$

$\begin{array}{lll}3.2 & \text { Polylogarithmic representation for algebraic alphabets } & 8\end{array}$

4 Elliptic integral sectors $\quad 11$

4.1 Sector $I_{1,1,0,1,1,1,1,0,0}^{A} \quad 11$

$\begin{array}{ll}4.2 & \text { Solution of the second order differential equation } \\ & 13\end{array}$

4.3 Auxiliary bases and solution in terms of two-fold iterated integrals 14

$\begin{array}{ll}4.4 \text { Sector } I_{1,1,1,1,1,1,1,0,0}^{A} & 15\end{array}$

$\begin{array}{llr}5 & \text { The class of functions } & 18\end{array}$

$\begin{array}{llr}6 & \text { Conclusion and perspectives } & 19\end{array}$

$\begin{array}{ll}\text { A Integral basis } & 20\end{array}$

$\begin{array}{ll}\text { B Pre-canonical master integrals } & 30\end{array}$

$\begin{array}{ll}\text { C Alphabet } & 33\end{array}$

$\begin{array}{ll}\text { D Weight-two functions } & 34\end{array}$

$\begin{array}{lll}\text { E One-fold integral representations } & 40\end{array}$

$\begin{array}{lll}\text { F Maximal cut of the elliptic sectors } & 41\end{array}$

\section{Introduction}

At the Large Hadron Collider ( $\mathrm{LHC}$ ), the main production mode of the Standard Model (SM) Higgs boson is via gluon-gluon fusion. The Higgs boson does not couple directly to the gluons, the interaction being mediated by a heavy-quark loop. That makes the evaluation of the radiative corrections to Higgs boson production via gluon-gluon fusion challenging, since the Born process is computed through one-loop diagrams, the next-toleading order (NLO) QCD corrections involve the computation of two-loop diagrams, the next-to-next-to-leading order (NNLO) corrections the computation of three-loop diagrams, and so on. In fact, fully inclusive Higgs production is known up to NLO [1, 2], while Higgs 
production in association with one jet [3] and the Higgs $p_{T}$ distribution [4] are known only at leading order.

The evaluation of the radiative corrections simplifies considerably in the Higgs effective field theory (HEFT), where the heavy quark is integrated out and the Higgs boson couples directly to the gluons, effectively reducing the computation by one loop. For fully inclusive Higgs production, the HEFT is valid when the Higgs mass is smaller than the heavy-quark mass, $m_{H} \lesssim m_{Q}$. Thus it is expected to be a good approximation to the full theory (FT), which gets corrections from the top-mass contribution and from the top-bottom interference. In fact, using the FT NLO computation as a benchmark, one can see that the HEFT NLO computation approximates very well the FT NLO computation, since the top-bottom interference and the top-mass corrections are about the same size although with opposite sign [5]. At NNLO, the FT mass corrections are expected to be in the percent range, which is though competitive with the precision of the HEFT computation at next-to-next-to-next-to-leading order $\left(\mathrm{N}^{3} \mathrm{LO}\right)[6,7]$.

For Higgs production in association with one jet or for the Higgs $p_{T}$ distribution, using the leading-order results $[3,4]$ as a benchmark one can show that the HEFT is valid when $m_{H} \lesssim m_{Q}$ and the jet or Higgs transverse momenta are smaller than the heavy-quark mass, $p_{T} \lesssim m_{Q}[8,9]$. In the HEFT, Higgs production in association with one jet $[10,11]$ and the Higgs $p_{T}$ distribution [12] are known at NNLO. No complete FT results are known beyond the leading order. Approximate NLO top-mass effects have been computed, and shown to be small and to agree well with the HEFT for $p_{T} \lesssim m_{\text {top }}[13-15]$ and up to $p_{T} \sim 300 \mathrm{GeV}[16]$. However, they are expected to be non-negligible in the high $p_{T}$ tail. Finally, it is worth noting that in many New Physics (NP) models, the high $p_{T}$ tail of the Higgs $p_{T}$ distribution is sensitive to modifications of the Higgs-top coupling [17-19].

In this paper, we report on the analytic computation of all the planar master integrals which are needed to compute the two-loop scattering amplitudes for Higgs $\rightarrow 3$ partons, with full heavy-quark mass dependence. These are relevant to compute the FT NNLO corrections to fully inclusive Higgs production and the FT NLO corrections to Higgs production in association with one jet or to the Higgs $p_{T}$ distribution.

The differential equations method [20-24] has proven to be one of the most powerful tools to compute (dimensionally regularized) loop Feynman integrals. In particular, the reduction of the Feynman integrals to a set of linearly independent integrals, dubbed master integrals [25-28], through integration-by-parts identities, the exploration of new classes of special functions such as multiple polylogarithms [29, 30], and a better understanding of their functional properties [31-33], have made the technique increasingly efficient. However, until recently the method was mostly applied in relatively simple kinematic situations, with the Feynman integrals depending on few scales, while complicated integrals needed a caseby-case analysis.

A major breakthrough was made in [34], where a canonical form of the differential equations for Feynman integrals was proposed. A key idea is that the canonical basis can be found by inspecting the singularity structure of the loop integrand. More precisely, one computes the leading singularities, i.e. maximal multidimensional residues of the loop integrand $[35,36]$. The fact that this can be done before the differential equations are set up 
renders this technique extremely efficient. ${ }^{1}$ When considering differential equations for a set of integrals defined to be pure functions of uniform weight, all relevant information about the analytic properties of the result is manifest at the level of the equations. Moreover, it is possible to find an analytic expression for the master integrals in terms of iterated integrals over algebraic kernels in a fully algorithmic way, up to any order of the dimensional regularization parameter (see [39-55] for many applications of these ideas). It is important to note that these ideas also streamline calculations whose output cannot be immediately written in terms of multiple polylogarithms, but where Chen iterated integrals [56] are the appropriate special functions, see e.g. [51, 57]. This class of functions will also be important in this paper.

Beyond Chen iterated integrals, there are cases where elliptic integrals appear. This is typically related to several equations being coupled in four dimensions, see e.g. [57, 58]. The appearance of elliptic integrals can be also anticipated by inspecting the maximal cuts of the corresponding loop integrands [59]. In this case the precise form of the canonical basis is not yet known, and presumably finding it will involve a generalization of the concept of leading singularities.

Over the last two decades a lot of effort has been made to understand the analytic properties of Feynman integrals which go beyond the multiple polylogarithms case, mostly related to the so-called sunrise diagram [38, 60-69]. However, to the best of our knowledge, such a generalized class of Feynman integrals has not been used so far in a fully analytic computation of a four-point multiscale scattering amplitude. In this paper, we compute in the Euclidean region all the planar master integrals relevant for Higgs $\rightarrow 3$ partons, retaining the full heavy-quark mass dependence, which include two elliptic integral sectors.

We write down the differential equations following the approach of [34]. We find that most integrals can be expressed in terms of Chen iterated integrals [56]. The corresponding function alphabet depends on three dimensionless variables and contains 49 letters, underlining the complexity of the problem. Having a fast and reliable numerical evaluation in mind, we derive a representation of all functions up to weight two in terms of logarithms and dilogarithms. Following [57], this allows us to write the weight-three and four functions in terms of one-fold integral representations. We find the latter suitable for numerical evaluation. We show that the two remaining integral sectors involve elliptic integrals. We analyze the corresponding system of coupled equations, and solve them in a suitable variable. An important tool is to reduce the problem to a one-variable problem (a similar strategy has been used in [70] to effectively rationalize the alphabet of multiscale processes). The solution at any order in $\epsilon$ can be expressed in terms of iterated integrals involving elliptic kernels. We then show that using auxiliary bases and basis shifts, the result for the elliptic sectors can be expressed in terms of two and three-fold iterated integrals, which we find suitable for numerical evaluation.

\footnotetext{
${ }^{1}$ An alternative approach to finding a canonical basis was proposed in [37-39]. It is based on the idea of transforming the system of differential equations such that the order of all singularities is manifest. In their current form, the ensuing algorithms require that the integrals depend in a rational way on a single variable, and usually yield rather complicated transformation matrices.
} 


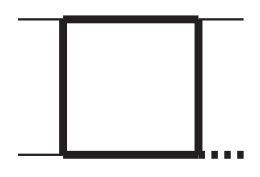

Figure 1. Four-denominator topology for the LO contribution to the cross section of Higgs boson production in association with a jet. Thick lines represent heavy quarks propagators. Thin lines represent massless external particles and propagators. The dashed external line represents the Higgs boson.

The outline of the paper is as follows. In section 2 we briefly discuss the reduction to the master integrals and the kinematics of the processes under consideration. In section 3 we review the differential equations method in the context of pure functions of uniform weight, i.e. the canonical basis approach. In section 3.2 we show that when a canonical basis exists the solution can be expressed to all orders of the dimensional regularization parameter in terms of multiple polylogarithms, also when a rational parametrization of the alphabet is not possible. We derive a one-fold integral representation of the result up to weight four which is suitable for fast and reliable numerical evaluation. In section 4 we discuss in detail how to analytically solve the elliptic sectors in terms of iterated integrals over elliptic kernels. In section 5 we discuss the class of functions used to represent the elliptic sectors. In section 6 we conclude and discuss future directions. We also provide six appendices in which we collect more details about the calculation. In appendix A we write the explicit expressions for the canonical form of the master integrals, or conversely for the basis choice in the elliptic case. In appendix B we show the 125 master integrals in the pre-canonical form. In appendix $\mathrm{C}$, we give the alphabet for the master integrals. In appendix D we list the dilogarithms we used to express the master integrals at weight two. In appendix $\mathrm{E}$ we give more details about the one-fold integral representation in terms of which we express the master integrals not depending on elliptic integrals. Finally in appendix $\mathrm{F}$ we show that the maximal cut of the six-denominator elliptic sector provides useful information about the class of functions which characterise the sector.

\section{Notations and conventions}

The leading order QCD contribution to Higgs decay to three partons, or alternatively to Higgs production in hadronic collisions, is a process mediated by a loop of heavy quarks. This is due to the fact that the SM Higgs boson does not couple directly to massless particles. The decay channels are $H \rightarrow g g g$ and $H \rightarrow g q \bar{q}$; the production channels are $g g \rightarrow g H, g q \rightarrow q H$ and $q \bar{q} \rightarrow g H$. The one-loop Feynman diagrams for all these processes can be described using the four-denominator topology ${ }^{2}$ (or sector) depicted in figure 1.

At NLO in $\alpha_{S}$, Feynman diagrams with up to seven propagators contribute to the processes above. They can all be described using the eight different planar seven-propagator

\footnotetext{
${ }^{2} \mathrm{~A}$ topology is composed of the integrals for which the same set of propagators have positive powers, while a subtopology (or subsector) is a set of integrals for which the propagators with positive powers are a subset of the ones of a given topology.
} 

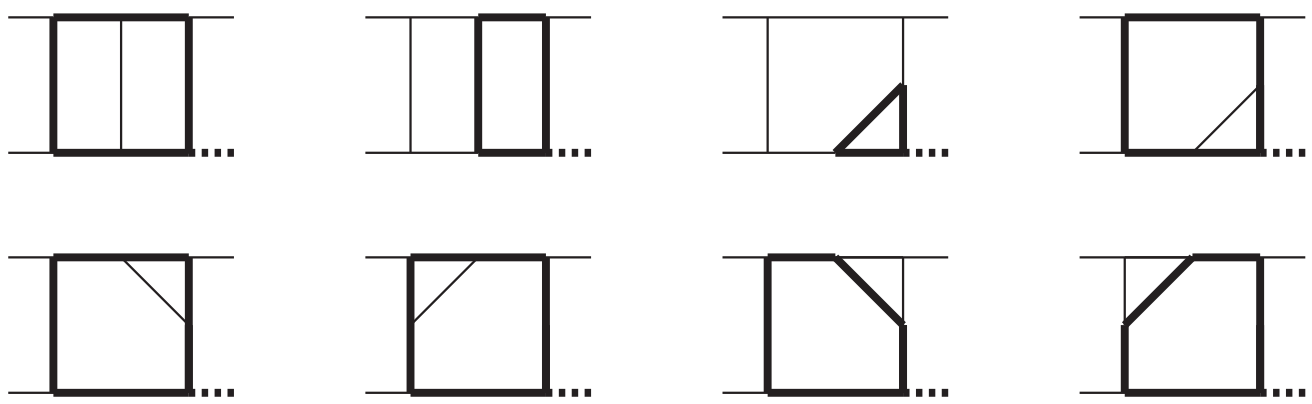

Figure 2. Planar seven-denominator topologies for the NLO contribution to the cross section of Higgs boson production in association with a jet in proton collisions, with full heavy-quark mass dependence.

topologies (and their subtopologies) depicted in figure 2. We parametrized all eight topologies into nine-propagator integral families and we reduced the corresponding dimensionally regularized integrals to a minimal set of independent integrals, dubbed master integrals, using the computer program FIRE [71-73] combined with LiteRed [74]. The list of denominators defining the integral families and additional details about this part of the calculation are provided in appendix A.

The most general integral is defined in $D=4-2 \epsilon$ space-time dimensions as,

$$
I_{a_{1}, a_{2}, a_{3}, a_{4}, a_{5}, a_{6}, a_{7}, a_{8}, a_{9}}^{i}=\int \frac{d^{D} k_{1} d^{D} k_{2}}{i \pi^{D / 2} i \pi^{D / 2}} \frac{\left[d_{8}^{i}\right]^{-a_{8}}\left[d_{9}^{i}\right]^{-a_{9}}}{\left[d_{1}^{i}\right]^{a_{1}}\left[d_{2}^{i}\right]^{a_{2}}\left[d_{3}^{i}\right]^{a_{3}}\left[d_{4}^{i}\right]^{a_{4}}\left[d_{5}^{i}\right]^{a_{5}}\left[d_{6}^{i}\right]^{a_{6}}\left[d_{7}^{i}\right]^{a_{7}}},
$$

where $i$ is the family index, and $a_{i}$ are integers. The reduction process leads to a set of 125 master integrals, shown in figure 3, that may be of relevance to more than one physical process. We shall focus here on a Higgs boson decaying to three partons and on Higgs + jet production. These processes differ by the physical phase-space region. Defining,

$$
s=\left(p_{1}+p_{2}\right)^{2}, \quad t=\left(p_{1}+p_{3}\right)^{2}, \quad u=\left(p_{2}+p_{3}\right)^{2}, \quad p_{4}^{2}=s+t+u,
$$

where $p_{1}^{2}=p_{2}^{2}=p_{3}^{2}=0$, the relevant physical regions are

$$
H \text { decay : } s>0, t>0, u>0, \quad H+\text { jet }: s>p_{4}^{2}>0, t<0, u<0,
$$

both with the internal quark mass $m^{2}>0$. The integrals are functions of three dimensionless invariants,

$$
x=\left\{x_{1}, x_{2}, x_{3}\right\},
$$

with

$$
x_{1}=\frac{s}{m^{2}}, \quad x_{2}=\frac{p_{4}^{2}}{m^{2}}, \quad x_{3}=\frac{t}{m^{2}} .
$$

In this paper we evaluate the integrals in the Euclidean region where no branch cuts are present, or rather in the subset there-of which has,

$$
x_{3}<x_{2}<x_{1}<0 .
$$




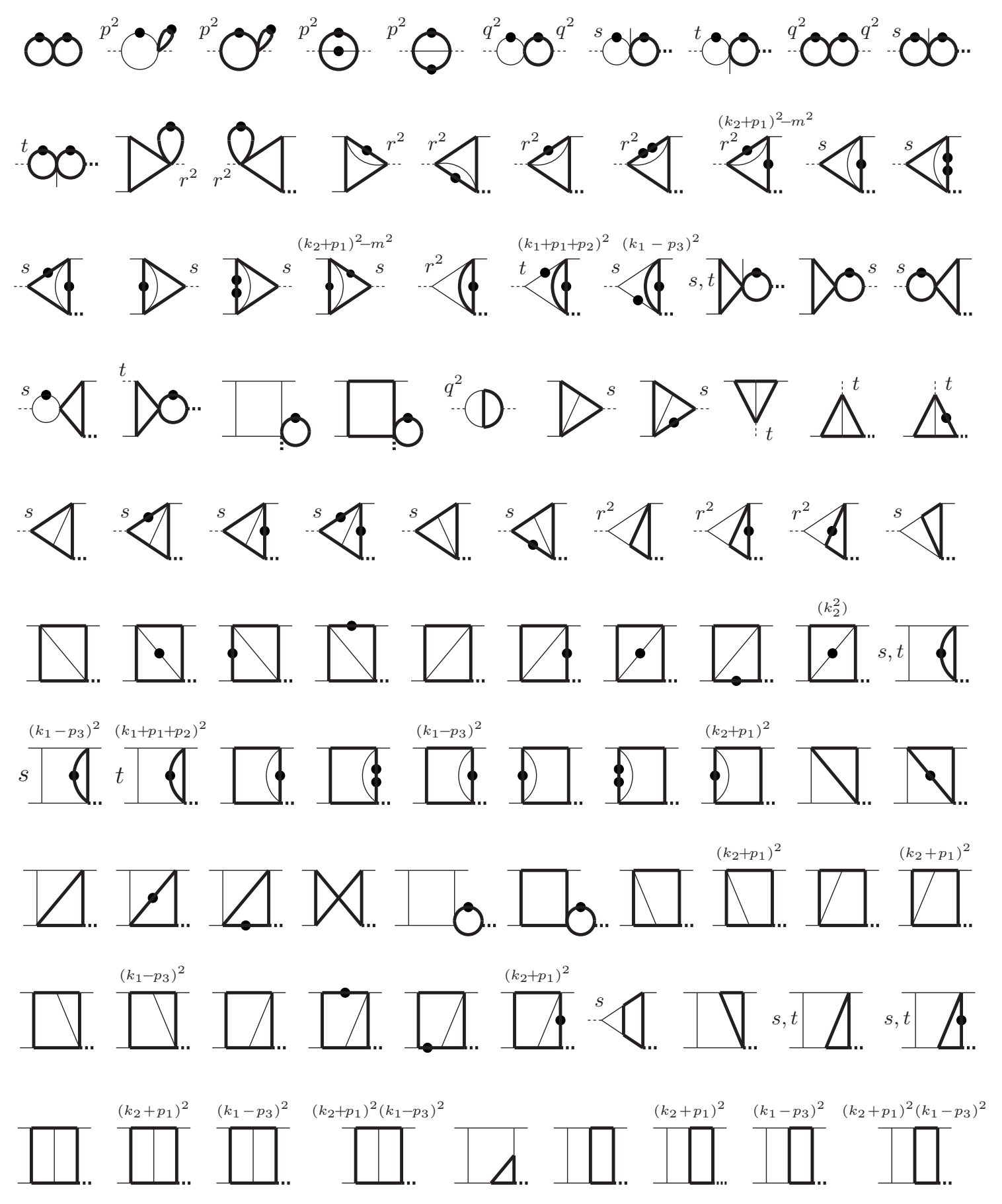

Figure 3. Master integrals in pre-canonical form. Internal plain thin lines represent massless propagators, while thick lines represent the top propagator. External plain thin lines represent massless particles on their mass-shell. External dashed thin lines represent the dependence on $s$, $t$, or $m_{H}^{2}$. The external dashed thick line represents the Higgs on its mass-shell. The squared momentum $p^{2}$ can assume the values $p^{2}=s, t, m_{H}^{2}$. The squared momentum $q^{2}$ can assume the values $q^{2}=s, m_{H}^{2}$. The squared momentum $r^{2}$ can assume the values $r^{2}=s, t$. 
It is then possible to analytically continue the result to the physical region using the Feynman prescription, by assigning a positive infinitesimal imaginary part to the external invariants and a negative infinitesimal imaginary part to the internal masses. The analytic continuation of the master integrals will be provided elsewhere.

The full basis of master integrals we evaluated in this paper is listed in appendix A.

The explicit results for the master integrals require about $200 \mathrm{MB}$ to be stored in electronic form, and can be obtained upon request to the authors.

\section{Differential equations}

In order to analytically compute the master integrals we rely on the differential equations method [20-24]. All the integrals discussed in this paper can be expressed in terms of multiple polylogarithms except eight of them, which involve elliptic integrals. In the polylogarithmic case we find a modified basis of integrals that are pure functions of uniform weight [34]. In this basis the differential equations take a canonical form and can be readily solved. This basis is found by choosing integrals with constant leading singularities. In the elliptic case the appropriate generalization of the notion of leading singularity has not yet been worked out. It is nevertheless possible to choose a basis where the elliptic nature of the integrals is manifest and the problem can be reduced to the solution of second order differential equations, as we discuss in section 4.

\subsection{General features of differential equations for Feynman integrals}

Denoting a set of $N$ basis integrals by $f$, the set of kinematical variables by $x$, and working in $D=4-2 \epsilon$ dimensions, it is possible to define a system of first order linear differential equations for the integrals, that can be written in total generality as,

$$
\partial_{m} f(x, \epsilon)=A_{m}(x, \epsilon) f(x, \epsilon)
$$

where we used the shorthand $\partial_{m}=\partial / \partial x_{m}$, and $A_{m}(x, \epsilon)$ is an $N \times N$ matrix with rational entries of its variables. The matrix $A_{m}(x, \epsilon)$ satisfies the integrability condition,

$$
\partial_{n} A_{m}-\partial_{m} A_{n}-\left[A_{n}, A_{m}\right]=0
$$

where $\left[A_{n}, A_{m}\right]=A_{n} A_{m}-A_{m} A_{n}$.

The choice of the basis is not unique. Performing a basis change $f \rightarrow B f$ the system of differential equations transforms according to

$$
A_{m} \rightarrow B^{-1} \partial_{x_{m}} B-B^{-1} A_{m} B
$$

In [34] it was conjectured that performing a basis change with algebraic coefficients, for integral sectors expressible in terms of multiple polylogarithms, it is possible to factorize out the $\epsilon$ dependence of the differential equations,

$$
\partial_{m} f(x, \epsilon)=\epsilon A_{m}(x) f(x, \epsilon) .
$$


Such a system of differential equations is said to be in canonical form. In order to discuss the properties of the solution it is convenient to write the differential equations in differential form,

$$
d f(x, \epsilon)=\epsilon d \tilde{A}(x) f(x, \epsilon),
$$

where $\tilde{A}$ is a matrix such that,

$$
\frac{\partial \tilde{A}(x)}{\partial x_{m}}=A_{m}(x) .
$$

The matrix elements of $\tilde{A}(x)$ are $\mathbb{Q}$-linear combinations of logarithms. The arguments of the logarithms are known as letters, while the set of linearly independent letters is known as alphabet. The main virtue of the canonical system of differential equations is that its solution is elementary, and it can be written for general $\epsilon$ in terms of a path-ordered exponential,

$$
f(x, \epsilon)=P e^{\epsilon \int_{C} d \tilde{A}} f(0, \epsilon),
$$

where $P$ is the path ordering operator along the integration path $C$, connecting the boundary point to $x$, while $f(0, \epsilon)$ are boundary conditions for $f(x, \epsilon)$. The solution can be expressed as a power series around $\epsilon=0$. Denoting with $f^{(i)}(x)$ the coefficient of $\epsilon^{i}$, we have,

$$
f(x)=\sum_{i} f^{(i)}(x) \epsilon^{i}
$$

and the different orders of the solution are related by the following recursive relation,

$$
f^{(i)}(x)=\int_{C} d \tilde{A}(x) f^{(i-1)}+f^{(i)}(0) .
$$

The previous relation shows that the solution is expressed to all orders of $\epsilon$ in terms of Chen iterated integrals [56]. The solution is a pure function of uniform weight corresponding to the order of the $\epsilon$ expansion.

The specific choice of the integral basis leading to the canonical form was achieved using the ideas outlined in [34]. In particular, it is expected that integrals with constant leading singularities [36] satisfy canonical differential equations. Using generalized cuts we look for combinations of integrals with simple leading singularities, that can be normalized to unity rescaling the candidate integrals. This typically leads to a form close to the canonical form. The remaining unwanted terms can be then algorithmically removed from the differential equations shifting the integral basis $[42,44,57]$.

\subsection{Polylogarithmic representation for algebraic alphabets}

The alphabet (see appendix $\mathrm{C}$ for the explicit alphabet of the integral families) of the canonical integrals discussed in this paper contains 8 independent square roots that cannot be simultaneously rationalized via a variable change. This means that it is not possible to directly integrate (3.9) in terms of multiple polylogarithms.

However we can find an expression in terms of these functions by making a suitable ansatz in terms of polylogarithms of a given weight. The main task is to find suitable function arguments, as we discuss presently. This strategy is streamlined using the concept 
of symbol $[29,31,75]$ of an iterated integral. The symbol corresponds to the integration kernels defining the iterated integrals. Since the integral basis is chosen to be of uniform weight, the symbol of the solution is completely manifest in our differential equations approach. Denoting by $f_{n}^{(i)}$ the $n^{\text {th }}$ component of the basis at $\mathcal{O}\left(\epsilon^{i}\right)$, and by $\tilde{A}_{n m}$ the $n^{\text {th }}$-row, $m^{\text {th }}$-column entry of matrix $\tilde{A}$, we have the following expression for the symbol of $f_{n}^{(i)}$,

$$
\mathcal{S}\left(f_{n}^{(i)}(x)\right)=\sum_{m} \mathcal{S}\left(f_{m}^{(i-1)}(x)\right) \otimes \mathcal{S}\left(\tilde{A}_{n m}(x)\right) .
$$

The corresponding polylogarithmic functions can be found proceeding in the following algorithmic steps (see also [31, 32]).

1. One generates a list of function arguments as monomials in the letters appearing in the alphabet of eq. (3.10). For the classical polylogarithms $\operatorname{Li}_{n}(x)$, one requires that $1-x$ factorizes over the alphabet. (A caveat is that in principle spurious letters might be needed [32].) For $\operatorname{Li}_{2,2}(x, y)$, the condition is that $1-x, 1-y, 1-x y$ factorize over the alphabet. Similar factorization properties are required for higher weight functions.

When square roots are present it might be difficult to directly check factorization over the alphabet. In practice we can proceed as follows. We consider the logarithm of the function argument whose factorization we want to check, and we equate it to a generic linear combination of the logarithms of the alphabet letters (ansatz). Since additive constants are irrelevant at the symbol level, we derive the identity with respect to each variable. We then specialize the resulting linear system of equations for the free coefficients of the ansatz to many numeric values of the variables. If a solution to the equations exists the argument factorizes as desired over the alphabet and the solution defines the factorized form.

2. For each weight, one chooses a maximal set of linearly independent functions from the set of functions generated at the previous step. The linear independence can be verified using the symbol. One then writes down the most general ansatz for a $\mathbb{Q}$-linear combination of these functions and products thereof, of weight $i$. The coefficients of the ansatz are then fixed imposing that the symbol of the ansatz equals the symbol (3.10).

3. We determine the terms in the kernel of the symbol at weight $i$ by writing the most general ansatz in terms of the lower weight functions, and solving the differential equations at $\mathcal{O}\left(\epsilon^{i}\right)$ for the free coefficients of the ansatz.

4. We recover transcendental additive constants imposing boundary conditions.

Note that no assumptions were made on the rationality of the alphabet letters, so that the above steps generalize the algorithm of [32] to algebraic cases. Note also that, as opposed to a purely symbol-based approach, using the knowledge of the differential equations and of the boundary conditions, the solution is fully determined. 
In practice the alphabet under consideration is quite large, and a reasonably fast computer implementation of the algorithm above up to weight four is challenging. We can nevertheless use the algorithm to reconstruct polylogarithmic functions up to weight two, for which the alphabet letters contributing to the result are a relatively small subset of the full alphabet. The full set of linearly independent dilogarithms for the four families is listed in appendix D.

Having a representation of the weight-two functions in terms of classical polylogarithms at hand is in fact very useful. As was shown in ref. [57], this can be used to write down useful one-dimensional integral representations for the remaining weight-three and weightfour functions.

Following [57], we use the Chen integral representation of the solution to write down a one-fold integral representation at weight three and four. Parametrizing the integration path $C$ with $\alpha \in[0,1],(3.9)$ translates to an iterated integral,

$$
f^{(i)}(x)=\int_{0}^{1}\left(\partial_{\alpha} \tilde{A}(\alpha)\right) f^{(i-1)}(\alpha) d \alpha+f^{(i)}(0)
$$

In this language when the weight-two functions are known analytically, the weight-three functions are one-fold integrals. Initially, the weight-four functions are two-fold iterated integrals of differentials of logarithms, and they can be converted to one-fold integrals integrating by parts (see appendix E for a detailed discussion). In particular, the weightthree functions are one-fold integrals over linear combinations of weight-two functions with algebraic coefficients, while the weight-four functions are expressed in two ways. The first consists of logarithms times one-fold integrals over linear combinations of weight-two functions, therefore a function of weight one times one of weight three. The other consists of a one-fold integral of weight-three functions, that are expressed as a product of weight-two functions times logarithms, with algebraic coefficients.

The boundary conditions required to fix the solution are determined using the regularity of the pre-canonical integrals and the behavior of the algebraic factors defining the canonical basis in the boundary point. We find it convenient to use the boundary point $x_{1}=x_{2}=x_{3}=0$. The values of our integrals at this point correspond to the large heavyquark limit so that one can apply the corresponding well-known graph theoretical prescriptions [76-78]. In the limit all the canonical integrals vanish except those that factor into products of one-loop integrals (these integrals are known analytically to all orders $[24,79]$ ). With this choice of the boundary point we can parametrize the integration path as,

$$
x(\alpha)=\left\{x_{1} \alpha, x_{2} \alpha, x_{3} \alpha\right\},
$$

with $\alpha \in[0,1]$.

We have validated the analytic expressions performing numerical checks against the computer program FIESTA [80-82] for randomly selected points in the Euclidean region (2.6). 

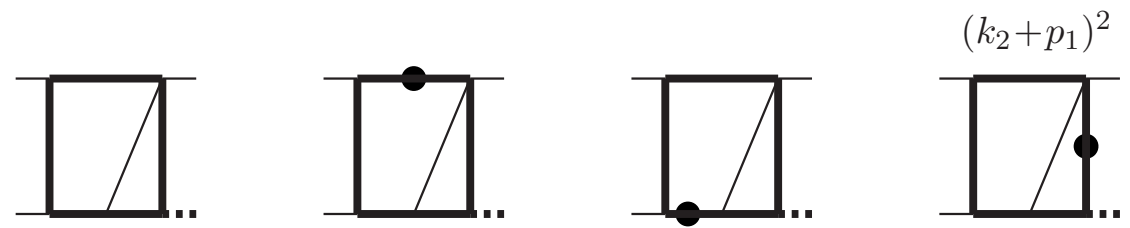

Figure 4. The four master integrals of the elliptic sector $I_{1,1,0,1,1,1,1,0,0}^{A}$.

\section{Elliptic integral sectors}

The last two integral sectors of Family A (see appendix A), integrals $f_{66}^{A}-f_{73}^{A}$, turn out to be expressed in terms of elliptic integrals. Using the language of the differential equations, the homogeneous part for sector $I_{1,1,0,1,1,1,1,0,0}^{A}$ is not cast in canonical form, as the solution is expressed in terms of complete elliptic integrals. In appendix $\mathrm{F}$ we show that these properties can be verified a priori analyzing the maximal cut of the integrals. In section 4.1 we show that we can reduce the problem to the solution of a second order differential equation. In section 4.2 we show that using a proper unidimensional parametrization of the integrals the relevant second order differential equation can be solved with elementary techniques. In section 4.3 we show that employing two auxiliary bases we obtain a two-fold iterated integral representation of the integral sector.

The highest sector of Family $\mathrm{A}$ is $I_{1,1,1,1,1,1,1,0,0}^{A}$. In this case the homogeneous part of the differential equations can be cast in canonical form, however they depend via inhomogeneous terms on the lower elliptic sector. In section 4.4 we write the result as a three-fold integral. We found these integral representations to be suitable for precise and reliable numerical evaluations. When implemented in Mathematica the evaluation of both the elliptic sectors in one Euclidean point takes about 10 minutes using one CPU, with about eight-digit accuracy. On the other hand the numerical evaluation of the full set of planar master integrals takes about 20 minutes.

\subsection{Sector $I_{1,1,0,1,1,1,1,0,0}^{A}$}

The integral sector $I_{1,1,0,1,1,1,1,0,0}^{A}$ has four master integrals, shown in figure 4 , which are expressed in terms of elliptic integrals, although its subtopologies do not involve them. We start by considering the following basis of finite integrals,

$$
\begin{aligned}
& h_{1}(x, \epsilon)=\epsilon^{4}\left(-x_{1}\right)^{3 / 2} I_{1,1,0,1,1,1,1,0,0}^{A}, \\
& h_{2}(x, \epsilon)=\epsilon^{4} I_{2,1,0,1,1,1,1,0,0}^{A}, \\
& h_{3}(x, \epsilon)=\epsilon^{3} I_{1,1,0,1,1,1,2,0,0}^{A}, \\
& h_{4}(x, \epsilon)=\epsilon^{4} I_{1,1,0,1,1,1,1,0,-1}^{A} .
\end{aligned}
$$

We parametrize the integrals through the linear parametrization (3.12), and we define the differential equations with respect to the new parameter using the chain rule,

$$
\partial_{\alpha} h(x(\alpha), \epsilon)=\sum_{i=1}^{3} x_{i} \partial_{x_{i}} h(x(\alpha), \epsilon),
$$


where $h$ is a vector, whose components are given in eq. (4.1). The differential equations have the following form,

$$
\partial_{\alpha} h(\alpha, \epsilon)=C^{(0)}(\alpha) h(\alpha, \epsilon)+\epsilon C^{(1)}(\alpha) h(\alpha, \epsilon)+\epsilon D^{(1)}(\alpha) g(\alpha, \epsilon)+\mathcal{O}\left(\epsilon^{2}\right),
$$

where $g(\alpha, \epsilon)$ is the vector of the subtopologies, $C^{(0)}(\alpha)$ and $C^{(1)}(\alpha)$ are $4 \times 4$ matrices and $D^{(1)}(\alpha)$ is a $4 \times 65$ matrix. In particular, the matrix $C^{(0)}(\alpha)$ has the form,

$$
C^{(0)}(\alpha)=\left(\begin{array}{cccc}
a_{1,1} & a_{1,2} & 0 & 0 \\
a_{2,1} & a_{2,2} & 0 & 0 \\
a_{3,1} & a_{3,2} & a_{3,3} & 0 \\
a_{4,1} & a_{4,2} & 0 & a_{4,4}
\end{array}\right) .
$$

The last two integrals are decoupled from each other, but this is not required for the applicability of the method described here. It is manifest that the equations for the first two integrals are coupled.

We look for a solution in power series around $\epsilon=0$,

$$
h(\alpha, \epsilon)=\sum_{i} h^{(i)}(\alpha) \epsilon^{i} .
$$

The coefficients of the power series satisfy the following first order differential equations,

$$
\partial_{\alpha} h^{(i)}(\alpha)=C^{(0)}(\alpha) h^{(i)}(\alpha)+C^{(1)}(\alpha) h^{(i-1)}(\alpha)+D^{(1)}(\alpha) g^{(i-1)}(\alpha)+\ldots
$$

where $h^{(i)}(\alpha)$ is the unknown and the other terms define the inhomogeneous part. A twoby-two system of first order differential equations for the first two components of $h(\alpha)$ defines a second order differential equation for the first component,

$$
\partial_{\alpha}^{2} h_{1}^{(i)}(\alpha)+p_{1}(\alpha) \partial_{\alpha} h_{1}^{(i)}(\alpha)+q_{1}(\alpha) h_{1}^{(i)}(\alpha)=r_{1}^{(i)}(\alpha),
$$

where $p_{1}(\alpha)$ and $q_{1}(\alpha)$ depend on the matrix elements of $C^{(0)}(\alpha)$, and are the same for every $i$, while $r_{1}^{(i)}(\alpha)$ is a function of the inhomogeneous part of (4.6). Once two homogeneous solutions of (4.7), $y_{1}(\alpha)$ and $y_{2}(\alpha)$, have been found, a particular solution can be determined using the method of the variation of constants. In general we get,

$$
h_{1}^{(i)}(\alpha)=c_{1} y_{1}(\alpha)+c_{2} y_{2}(\alpha)-y_{1}(\alpha) \int_{0}^{\alpha} d z \frac{r_{1}^{(i)}(z)}{w(z)} y_{2}(z)+y_{2}(\alpha) \int_{0}^{\alpha} d z \frac{r_{1}^{(i)}(z)}{w(z)} y_{1}(z),
$$

where the arbitrary constants $c_{i}$ are fixed by the boundary conditions, and where $w(\alpha)$ is the Wronskian of the homogeneous solutions,

$$
w(\alpha)=y_{2}(\alpha) \partial_{\alpha} y_{1}(\alpha)-y_{1}(\alpha) \partial_{\alpha} y_{2}(\alpha)
$$

Once $h_{1}^{(i)}(\alpha)$ is solved, we can determine the remaining components of $h^{(i)}(\alpha)$. From (4.4) it follows that $h_{2}^{(i)}(\alpha)$ can be obtained from $h_{1}^{(i)}(\alpha)$ and its first derivative. In this way the expression of $h_{2}^{(i)}(\alpha)$ involves the same number of repeated integrations as $h_{1}^{(i)}(\alpha)$. In order to solve the last two integrals we solve the respective first order differential 
equations, which depend on $h_{1}^{(i)}(\alpha)$ and $h_{2}^{(i)}(\alpha)$ via the inhomogeneous terms. This shows that, when computed in this way, $h_{3}^{(i)}(\alpha)$ and $h_{4}^{(i)}(\alpha)$ involve one more repeated integration than $h_{1}^{(i)}(\alpha)$ and $h_{2}^{(i)}(\alpha)$. In order to optimize the numerical evaluation it is important to get rid of the extra integration. Furthermore, since at $\mathcal{O}\left(\epsilon^{4}\right)$ these integrals would be expressed in terms of five iterated integrations, one integration must be spurious. In the non-elliptic case one is able to remove extra integrations using integration by parts. However in the elliptic case in order to perform an integration by parts one needs to integrate over elliptic integrals, which is in general not possible analytically. We show how this is done in section 4.3 .

\subsection{Solution of the second order differential equation}

The possibility of solving algorithmically a second order differential equation is related to the number of its singular points, including the point at infinity. If there are up to three singular points the equation can be cast in the form of the hypergeometric equation and two linearly independent solutions can be expressed in terms of hypergeometric functions [83]. Similar algorithms exist when four singular points are present. On the other hand if more than four singular points are present the solution requires a case by case analysis.

After differentiating with respect to the Mandelstam variables, the second order differential equation for $I_{1,1,0,1,1,1,1,0,0}^{A}$ has six singular points. We show that using the parametrization (3.12) the solution can be reduced to the three singular point case.

Once $h_{1}(x(\alpha), \epsilon)$ is made explicit as in (4.1), the coefficients of the second order differential equation (4.7) are,

$$
p_{1}(\alpha)=\frac{2 x_{1}\left(\alpha x_{1}\left(x_{2}-x_{3}\right)^{2}-4\left(x_{2}\left(x_{1}-x_{3}\right)+x_{3}\left(x_{1}+x_{3}\right)\right)\right)}{d_{1}(\alpha)},
$$

and,

$$
q_{1}(\alpha)=\frac{x_{1}^{2}\left(x_{2}-x_{3}\right)^{2}}{4 d_{1}(\alpha)}
$$

where,

$$
d_{1}(\alpha)=x_{1}^{2} \alpha^{2}\left(x_{2}-x_{3}\right)^{2}-8 x_{1} \alpha\left(x_{2}\left(x_{1}-x_{3}\right)+x_{3}\left(x_{1}+x_{3}\right)\right)+16\left(x_{1}+x_{3}\right)^{2} .
$$

We see that after using parametrization (3.12) we are left with three singular points, which are the two roots of $d_{1}(\alpha)=0$ and the point at infinity. The homogeneous solutions of (4.7) can be then readily found ${ }^{3}$ to be

$$
y_{1}(\alpha)=K\left(\frac{1}{2}-\frac{k(\alpha)}{2}\right), \quad y_{2}(\alpha)=K\left(\frac{1}{2}+\frac{k(\alpha)}{2}\right),
$$

where the function $k(z)$ is,

$$
k(z)=\frac{\left(x_{2}-x_{3}\right)^{2} x_{1} z-4\left(x_{2}\left(x_{1}-x_{3}\right)+x_{3}\left(x_{1}+x_{3}\right)\right)}{8 \sqrt{x_{1} x_{3} x_{2}\left(x_{1}+x_{3}-x_{2}\right)}},
$$

\footnotetext{
${ }^{3}$ We have found the Mathematica built-in function DSolve to be adequate. In alternative it is possible to use the algorithm of [83].
} 
and $K(z)$ is the complete elliptic integral of the first kind, ${ }^{4}$

$$
K(z)=\int_{0}^{1} \frac{d t}{\sqrt{\left(1-t^{2}\right)\left(1-z t^{2}\right)}} .
$$

The complete elliptic integral of the second kind is defined as,

$$
E(z)=\int_{0}^{1} \frac{\sqrt{1-z t^{2}}}{\sqrt{1-t^{2}}} d t
$$

We have the following relations for the derivatives of the complete elliptic integrals,

$$
\frac{d K(z)}{d z}=\frac{E(z)-(1-z) K(z)}{2(1-z) z},
$$

and,

$$
\frac{d E(z)}{d z}=\frac{E(z)-K(z)}{2 z} .
$$

Since $h_{2}^{(i)}(\alpha)$ is a linear combination of $h_{1}^{(i)}(\alpha)$ and its first derivative, it is expressed in terms of complete elliptic integrals of the first and second kind, of the same arguments as in (4.13). The Wronskian of the two homogeneous solutions is defined in terms of the derivatives above. Its expression is a rational function of the integration variable $\alpha$, and in our case it reads,

$$
w(\alpha)=\frac{4 \pi x_{1} \sqrt{x_{1} x_{3} x_{2}\left(x_{1}+x_{3}-x_{2}\right)}}{d_{1}(\alpha)} .
$$

This property can be proven by using the Legendre identity,

$$
E(z) K(1-z)+E(1-z) K(z)-K(z) K(1-z)=\frac{\pi}{2} .
$$

Thanks to the overall normalization factor we chose for $h_{1}(x, \epsilon)$, it is elementary to determine boundary conditions and use them to fix the free constants of the general solution (4.8). Integral $I_{1,1,0,1,1,1,1,0,0}^{A}$ is regular for $\alpha=0$, so that $h_{1}(0, \epsilon)=\partial_{\alpha} h_{1}(0, \epsilon)=0$ and $c_{1}=c_{2}=0$.

\subsection{Auxiliary bases and solution in terms of two-fold iterated integrals}

Since we need to evaluate the components of $h(4.1)$ through $\mathcal{O}\left(\epsilon^{4}\right)$, all the $I$ integrals of eq. (4.1) need to be computed through $\mathcal{O}\left(\epsilon^{0}\right)$, except $I_{1,1,0,1,1,1,2,0,0}^{A}$ which must be evaluated through $\mathcal{O}(\epsilon)$. Higher orders are irrelevant for two-loop processes. In general, the result for a master integral at $\mathcal{O}\left(\epsilon^{i}\right)$ is obtained integrating over subtopologies through $\mathcal{O}\left(\epsilon^{i-1}\right)$ and, if coupled to them, over integrals of the same topology at $\mathcal{O}\left(\epsilon^{i}\right)$. In section 3.2 we saw that weight-two functions can be expressed in terms of logarithms and dilogarithms, and weight-three functions can be reduced to one-fold integrals. This implies that, because of the general form of $(4.3), h_{3}^{(3)}(\alpha)$ is expressed in terms of one-fold integrals, while $h_{1}^{(4)}(\alpha)$

\footnotetext{
${ }^{4}$ Note that also a different convention exists for the definition of complete elliptic integrals such that, compared to our definition, the argument is replaced by its squared at the level of the integrand.
} 
and $h_{2}^{(4)}(\alpha)$ are expressed in terms of up to two-fold integrals. On the other hand $h_{3}^{(4)}(\alpha)$ and $h_{4}^{(4)}(\alpha)$ involve three-fold iterated integrals.

In order to avoid considering more than two iterated integrations we introduce two auxiliary bases, satisfying differential equations of the form of (4.3)-(4.4). The bases are defined in such a way that the respective second integrals are linearly independent of $h_{2}(x, \epsilon)$ and $h_{1}(x, \epsilon)$, and linearly independent on each other. Two bases satisfying these requests are,

$$
\left\{h_{1}(x, \epsilon), h_{5}(x, \epsilon), h_{3}(x, \epsilon), h_{4}(x, \epsilon)\right\}
$$

with,

$$
h_{5}(x, \epsilon)=\epsilon^{4} I_{1,2,0,1,1,1,1,0,0}^{A}
$$

and,

$$
\left\{h_{1}(x, \epsilon), h_{6}(x, \epsilon), h_{3}(x, \epsilon), h_{4}(x, \epsilon)\right\}
$$

with,

$$
h_{6}(x, \epsilon)=\epsilon^{4} I_{1,1,0,1,1,1,2,-1,0}^{A} .
$$

Both $h_{5}(x, \epsilon)$ and $h_{6}(x, \epsilon)$ are finite. Since the differential equations for basis $\left\{h_{1}(x, \epsilon)\right.$, $\left.h_{5}(x, \epsilon), h_{3}(x, \epsilon), h_{4}(x, \epsilon)\right\}$ and basis $\left\{h_{1}(x, \epsilon), h_{6}(x, \epsilon), h_{3}(x, \epsilon), h_{4}(x, \epsilon)\right\}$ have the form of (4.3)-(4.4), we can compute $h_{5}^{(4)}(x, \epsilon)$ and $h_{6}^{(4)}(x, \epsilon)$ as functions of $h_{1}^{(4)}(x, \epsilon)$ and its first derivative, as we did in section 4.1 for $h_{2}(x, \epsilon)$. In this way $h_{5}^{(4)}(x, \epsilon)$ and $h_{6}^{(4)}(x, \epsilon)$ are expressed as linear combinations of up to two-fold integrals.

The full (finite) basis for the integral sector is then chosen to be,

$$
\begin{aligned}
f_{66}^{A} & =h_{1}(x, \epsilon), \\
f_{67}^{A} & =\left(-x_{1}\right)^{3 / 2} x_{1} h_{2}(x, \epsilon), \\
f_{68}^{A} & =\left(-x_{1}\right)^{3 / 2} x_{1} h_{5}(x, \epsilon), \\
f_{69}^{A} & =\left(-x_{1}\right)^{3 / 2} x_{1} h_{6}(x, \epsilon) .
\end{aligned}
$$

With this choice all the integrals can be computed up to $\mathcal{O}\left(\epsilon^{4}\right)$ in terms of up to two-fold integrals. The algebraic prefactors are not strictly necessary but they lead to simpler expressions.

Interestingly, if we consider the differential equations for $f_{66}^{A}-f_{69}^{A}$, they are fully coupled and cannot be solved directly. We could nevertheless solve them with the help of auxiliary bases.

\subsection{Sector $I_{1,1,1,1,1,1,1,0,0}^{A}$}

The highest elliptic sector is $I_{1,1,1,1,1,1,1,0,0}^{A}$. It has four master integrals, shown in figure 5, and it depends on the elliptic subsector $I_{1,1,0,1,1,1,1,0,0}^{A}$ via inhomogeneous terms in the differential equations. Using the criteria outlined in [34] we can find a basis satisfying,

$$
\partial_{\alpha} v(\alpha, \epsilon)=\epsilon F^{(1)}(\alpha) v(\alpha, \epsilon)+G^{(0)}(\alpha) g(\alpha, \epsilon)+\epsilon G^{(1)}(\alpha) g(\alpha, \epsilon)+\mathcal{O}\left(\epsilon^{2}\right) .
$$




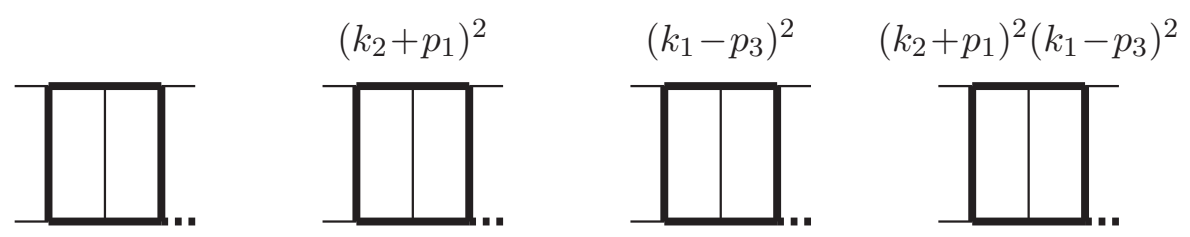

Figure 5. The four master integrals of the elliptic sector $I_{1,1,1,1,1,1,1,0,0}^{A}$.

$v(\alpha, \epsilon)$ is a four-dimensional basis vector for the highest elliptic sector, $g(\alpha, \epsilon)$ is the vector of the subtopologies, $F^{(1)}(\alpha)$ is a $4 \times 4$ matrix, $G^{(0)}(\alpha)$ and $G^{(1)}(\alpha)$ are $4 \times 69$ matrices. The homogeneous part is in canonical form, while this is not the case for the subtopologies. When solving the above equation for a given power of $\epsilon$, we have to integrate over subsectors of the same order due to the $G^{(0)}(\alpha)$ matrix. For numerical optimization it is convenient to get rid of such integrals. Matrix elements of $G^{(0)}(\alpha)$ corresponding to non-elliptic subsectors are removed with a basis shift, as described in $[44,57]$. In order to remove $G^{(0)}(\alpha)$ entries corresponding to elliptic subsectors we proceed as follows. Let us consider the $i^{\text {th }}$ component of $v$, which fulfills the equation,

$$
\partial_{\alpha} v_{i}(\alpha, \epsilon)=\sum_{j=1}^{2} k_{i j}(\alpha) e_{j}(\alpha, \epsilon)+\mathcal{O}(\epsilon)
$$

where $k_{i j}(\alpha)$, with $i=1, \ldots, 4$ and $j=1,2$, are known algebraic functions and $e_{1}, e_{2}$ are two coupled integrals of an elliptic subsector, satisfying,

$$
\partial_{\alpha} e_{i}(\alpha, \epsilon)=\sum_{j=1}^{2} a_{i j}(\alpha) e_{j}(\alpha)+\mathcal{O}(\epsilon) .
$$

We shift $v_{i}(\alpha, \epsilon)$ according to,

$$
v_{i}(\alpha, \epsilon) \rightarrow v_{i}(\alpha, \epsilon)+\sum_{j=1}^{2} b_{i j}(\alpha) e_{j}(\alpha, \epsilon),
$$

where $b_{i j}(\alpha)$ are functions to be determined. After the basis shift the equation for $v_{i}$ reads,

$$
\partial_{\alpha} v_{i}(\alpha, \epsilon)=\sum_{j=1}^{2}\left(\partial_{\alpha} b_{i j}(\alpha)+\sum_{k=1}^{2} a_{k j}(\alpha) b_{i k}(\alpha)+k_{i j}(\alpha)\right) e_{j}(\alpha)+\mathcal{O}(\epsilon) .
$$

In order to remove terms proportional to $e_{1}(\alpha, \epsilon)$ and $e_{2}(\alpha, \epsilon)$, their coefficients must vanish, i.e. $b_{i j}(\alpha)$ must fulfill the equations,

$$
\partial_{\alpha} b_{i j}(\alpha)=-\sum_{k=1}^{2} a_{k j}(\alpha) b_{i k}(\alpha)-k_{i j}(\alpha)
$$

with $j=1,2$. For fixed $i$, the above equation is a two-by-two system of first order differential equations. The matrix defining the system is the transpose of the matrix defining (4.28). 
This implies that if $y_{1}(\alpha)$ and $y_{2}(\alpha)$ are the homogeneous solutions of (4.28) and $w(\alpha)$ is their Wronskian, the solutions of (4.31) are,

$$
c \frac{y_{1}(\alpha)}{w(\alpha)}, \quad c \frac{y_{2}(\alpha)}{w(\alpha)}
$$

where $c$ is an overall constant. Their Wronskian is $c^{2} / w(\alpha)$. Therefore with the method of the variation of constants the full expression for $b_{i 1}(\alpha)$ reads,

$$
b_{i 1}(\alpha)=-\frac{y_{1}(\alpha)}{w(\alpha)} \int_{1}^{\alpha} d t L_{i}(t) y_{2}(t)+\frac{y_{2}(\alpha)}{w(\alpha)} \int_{1}^{\alpha} d t L_{i}(t) y_{1}(t)
$$

where $L_{i}(\alpha)$ are functions of $k_{i 1}(\alpha)$ and $k_{i 2}(\alpha)$, and where two arbitrary integration constants have been set to zero. In addition, we set the lower integration bound to 1 but we have the freedom to choose a different value. Usually this is dictated by the properties of the integrand, that might have non-integrable singularities for specific integration bounds. Once $b_{i 1}(\alpha)$ is known it is elementary to obtain $b_{i 2}(\alpha)$ using the same differential equations.

For sector $I_{1,1,1,1,1,1,1,0,0}^{A}$ the integrals that need to be shifted are $f_{71}^{A}$ and $f_{73}^{A}$, as $e_{1}$ and $e_{2}$ defined via (4.29) are equal to $f_{66}^{A}$ and $f_{67}^{A}$ respectively. $y_{1}(\alpha)$ and $y_{2}(\alpha)$ are the same as those of (4.13) and,

$$
L_{2}(z)=\frac{x_{1}\left(x_{1}-x_{2}\right)}{\left(4-x_{1} z\right)^{3 / 2}}, \quad L_{4}(z)=\frac{x_{1}\left(x_{1}+x_{3}\right)}{\left(-x_{1} z\right)^{3 / 2}}
$$

while $L_{1}$ and $L_{3}$ vanish.

In general the integrals of (4.33) are not known analytically in closed form. Since after the basis shift they will contribute to the matrix elements of the differential equations, one might wonder if such a basis change is convenient in practice, as our main goal was to get rid of one integration. In practice, because of the simple form of (4.34), its numerical evaluation takes $\mathcal{O}\left(10^{-3}\right)$ sec. In this form the result for the elliptic sector at $\mathcal{O}\left(\epsilon^{4}\right)$ is in terms of three-fold integrals, while their numerical performance is comparable to the one of two-fold integrals. Alternatively, it is possible to series expand the complete elliptic integrals of eq. (4.33) and then perform the integrations analytically. ${ }^{5}$ In this way the result for the integral sector can be expressed in terms of two-fold integrals. ${ }^{6}$

\footnotetext{
${ }^{5}$ We series expand the complete elliptic integrals using well known results. The expansion around a generic point $z_{0}$ will involve powers of $z-z_{0}$, and factors of $\log \left(z-z_{0}\right)$ if $z_{0}$ is a singular point. It is then possible to perform the integrations analytically when considering the elementary functions of eq. (4.34).

${ }^{6}$ In order to get rid of the extra integration, one could have performed an integration by parts after solving directly eq. (4.26). Also this method introduces integrals over complete elliptic integrals and algebraic functions in the integrands of the solution. However such integrals are not as simple as the ones introduced by the basis shift, and the integration over the series expanded complete elliptic integrals is not straightforward.
} 


\section{The class of functions}

In order to discuss the general structure of the solution of sector $I_{1,1,0,1,1,1,1,0,0}^{A}$ let us introduce the following shorthands for the complete elliptic integrals defined in section 4.2,

$$
\begin{array}{ll}
K^{(1)}(\alpha)=K\left(\frac{1}{2}+\frac{k(\alpha)}{2}\right), & K^{(-1)}(\alpha)=K\left(\frac{1}{2}-\frac{k(\alpha)}{2}\right), \\
E^{(1)}(\alpha)=E\left(\frac{1}{2}+\frac{k(\alpha)}{2}\right), & E^{(-1)}(\alpha)=E\left(\frac{1}{2}-\frac{k(\alpha)}{2}\right) .
\end{array}
$$

Integrals $f_{66}^{A,(4)}-f_{69}^{A,(4)}$ are expressed as linear combinations of the class of functions,

$$
\mathcal{E}^{(\sigma)}(1) \int_{0}^{1} \mathcal{F}(t) \mathcal{E}^{(-\sigma)}(t) d t
$$

where $\mathcal{E}^{(\sigma)}$ can be one of the following complete elliptic integrals,

$$
K^{(\sigma)}(\alpha), \quad E^{(\sigma)}(\alpha)
$$

where $\sigma \in\{-1,1\} . \mathcal{F}(t)$ denotes a linear combination of pure weight-two and weight-three functions, belonging to the subtopologies, multiplied by either derivatives of logarithms or derivatives of algebraic functions, with respect to $\alpha .{ }^{7}$ Interestingly, weight-three functions are never multiplied by derivatives of logarithms, but only by the following simple inverse square roots (modulo functions depending only on rescaled Mandelstam invariants),

$$
\frac{1}{\sqrt{\alpha}}, \quad \frac{1}{\sqrt{4-x_{1} \alpha}} .
$$

The same class of functions has been found in [84] for the massive crossed triangle. See $[62,63,67,85]$ for results in terms of elliptic polylogarithms [86], and $[64-66,69]$ for a related class of functions.

In order to decouple integral sector $I_{1,1,1,1,1,1,1,0,0}^{A}$ from sector $I_{1,1,0,1,1,1,1,0,0}^{A}$, in section 4.4 we performed a non-algebraic basis shift of $f_{71}^{A}$ and $f_{73}^{A}$, involving integrals of complete elliptic integrals, that we denote here with the following shorthands,

$$
\tilde{K}_{i}^{(1)}(\alpha)=\int_{1}^{\alpha} L_{i}(t) K^{(1)}(t) d t, \quad \tilde{K}_{i}^{(-1)}(\alpha)=\int_{1}^{\alpha} L_{i}(t) K^{(-1)}(t) d t,
$$

where $L_{i}(t)$ are those of eq. (4.34). For this reason the result for the highest elliptic sector is not directly expressed in terms of iterated integrals of the form of eq. (5.2), though such expressions can be immediately obtained by solving the differential equations without performing the non-algebraic basis shift. Integrals $f_{70}^{A,(4)}-f_{73}^{A,(4)}$ are linear combinations of polylogarithmic functions and of the class of functions,

$$
\int_{0}^{1} \mathcal{G}(t) \mathcal{E}^{(\sigma)}(t) \tilde{K}_{i}^{(-\sigma)}(t) d t
$$

\footnotetext{
${ }^{7}$ In a few cases also algebraic functions that are derivatives of (combinations of) incomplete elliptic integrals appear. However this result requires further investigation as a reparametrization of the square roots might reduce them to derivatives of algebraic or logarithmic functions.
} 
$\mathcal{G}(t)$ has the same properties as $\mathcal{F}(t)$ described above, but the prefactors of pure weightthree functions are any of the algebraic functions,

$$
\frac{1}{\sqrt{\alpha}}, \quad \frac{1}{\sqrt{4-x_{1} \alpha}}, \quad \sqrt{\alpha} .
$$

\section{Conclusion and perspectives}

In this paper we presented the analytic computation of all the planar master integrals which are necessary to evaluate the two-loop amplitudes for Higgs $\rightarrow 3$ partons, with the full heavy-quark mass dependence. They occur in the NNLO corrections to fully inclusive Higgs production and in the NLO corrections to Higgs plus one jet production in hadron collisions. The result is expressed in terms of iterated integrals over both algebraic and elliptic kernels. This is the first time that Feynman integrals for four-point multiscale amplitudes involving elliptic integrals are computed in a fully analytic way. While it was generally believed that the analytic computation of multiscale loop integrals with many internal massive lines was out of reach with present analytic tools, this work shows that new ideas involving the proper parametrization of the integrals, an optimal basis choice, and the subsequent solution with the differential equations method in terms of elliptic iterated integrals, are effective to treat such problems.

The computation of the non-elliptic integral sectors has been performed with the differential equations method applied to a set of basis integrals defined to be pure functions of uniform weight. The presence of many square roots that cannot be simultaneously rationalized makes the direct solution of these equations in terms of multiple polylogarithms not possible. We have shown that the Chen iterated integral representation plus the knowledge of the boundary conditions provide the information needed to integrate the system in terms of a minimal polylogarithmic basis, circumventing in this way the necessity to rationalize the square roots of the alphabet. To do so we used an algorithm for the integration of symbols with general algebraic alphabets, generalizing well established algorithms for the rational case.

We have seen that the crucial point for the computation of the elliptic sectors is the solution of the associated homogeneous second order differential equation. We noticed that a very simple univariate reparametrization of the integrals makes the equation elementary and standard tools are sufficient to solve it. The central point is that the fewer singular points are present in higher-order differential equations, the simpler is their solution. It will be important to further investigate and develop the idea of what is the proper parametrization of the integrals yielding the simplest singular structure of the equations. The univariate parametrization has also the benefit that only one set of differential equations has to be solved, while in the traditional approach one has to iteratively solve multiple sets of equations, one for each variable, which might be highly non-trivial when elliptic integrals are involved.

In contrast to the non-elliptic sectors, we did not use the notion of canonical basis for the elliptic sectors. Instead, we showed that the problem can be completely solved in total generality, once the relevant higher order homogeneous equations have been solved. 
However it will be important to extend the notion of canonical basis to elliptic cases. First, this will clarify the class of functions needed to represent the answer - in our case we used a rather general class that might still contain spurious information. Second, it is natural to expect that the explicit results for canonical integrals will be relatively compact. In order to define a canonical basis in the elliptic case, the notion of leading singularity has to be generalized, which is beyond the scope of the present paper (see appendix $\mathrm{F}$ for a discussion about the maximal cut of those integrals, which would be the starting point for defining a generalization of leading singularity in the elliptic case). In particular, we know [37-39] that it is possible to obtain a form of the differential equations with only Fuchsian singularities and linear in $\epsilon$. This is valid for any Feynman integral and it is another natural starting point for finding a canonical basis.

We showed that for the sake of stable and precise numerical evaluations we express the master integrals up to order $\epsilon^{4}$ in terms of one-fold integrals for the non-elliptic sectors, and up to three-fold integrals for the elliptic sectors. We found these representations suitable for numerical evaluation. In principle, as the integrands are known functions, it should be possible to achieve a series representation of the solution, though we did not attempt it as the integral representation already showed satisfying performance. It will be important to develop general purpose numerical routines for elliptic iterated integrals, so that one can take advantage of such analytic expressions also when higher loop orders are considered, i.e. when more iterated integrals are needed.

\section{Acknowledgments}

We would like to thank Claude Duhr and Yang Zhang for useful discussions and for reading parts of the manuscript. Part of the algebraic manipulations required in this work were carried out with FORM [87]. The Feynman diagrams were drawn with Axodraw [88]. VDD and HF were partly supported by the Research Executive Agency (REA) of the European Union, through the Initial Training Network LHCPhenoNet under contract PITN-GA-2010-264564. VDD and FM are supported by the Advanced ERC Grant Pert QCD. HF is supported through the Initial Training Network HiggsTools under contract PITN-GA-2012-316704. JMH is supported in part by a GFK fellowship and by the PRISMA cluster of excellence at Mainz university.

\section{A Integral basis}

In this appendix, we provide the explicit form of the integral families we used to parametrize the integrals defined in eq. (2.1). We call them family A, B, C, and D.

For each family, we perform an independent reduction to the master integrals. Then we perform a change of basis that maps the master integrals into the canonical form. We give such a canonical basis for each family separately. The canonical master integrals are labeled with $f_{n}^{i}$, with $i \in\{A, B, C, D\}$ and $n=1, \ldots, N$, where $N$ is the number of master integrals of the family under consideration. The elliptic sectors correspond to eight integrals of family A, labeled with $f_{66}^{A}-f_{73}^{A}$. These integrals are not in canonical form, as discussed in section 4 . 
For each family of integrals we define the corresponding system of differential equations, that we then solve as discussed in sections 3 and 4 .

Note that, in general, there is an overlap among the master integrals of the different families. Making the appropriate correspondences, we can reduce the process to the computation of 125 master integrals. In the next appendix, we draw these 125 (pre-canonical) master integrals and we link them to the corresponding canonical form.

We label with $p_{1}, p_{2}$, and $p_{3}$ the momenta of the massless partons, and with $p_{4}=$ $p_{1}+p_{2}+p_{3}$ the momentum of the Higgs. The loop momenta are labeled with $k_{1}$ and $k_{2}$. Finally, we use the shorthand $p_{i j}=p_{i}+p_{j}$.

Family A. Family A is defined by the nine propagators,

$$
\begin{array}{lll}
d_{1}^{A}=m^{2}-k_{1}^{2}, & d_{2}^{A}=m^{2}-\left(k_{1}+p_{12}\right)^{2}, & d_{3}^{A}=m^{2}-k_{2}^{2}, \\
d_{4}^{A}=m^{2}-\left(k_{2}+p_{12}\right)^{2}, & d_{5}^{A}=m^{2}-\left(k_{1}+p_{1}\right)^{2}, & d_{6}^{A}=-\left(k_{1}-k_{2}\right)^{2}, \\
d_{7}^{A}=m^{2}-\left(k_{2}-p_{3}\right)^{2}, & d_{8}^{A}=-\left(k_{2}+p_{1}\right)^{2}, & d_{9}^{A}=-\left(k_{1}-p_{3}\right)^{2},
\end{array}
$$

with the extra restriction that $a_{8}$ and $a_{9}$ are non-positive. The family contains 73 master integrals. Below, we give the basis transformation between pre-canonical and canonical forms.

$$
\begin{aligned}
f_{1}^{A}= & \epsilon^{2} I_{0,0,0,0,2,0,2,0,0}^{A} \\
f_{2}^{A}= & \epsilon^{2} x_{2} I_{0,2,0,0,0,1,2,0,0}^{A} \\
f_{3}^{A}= & \epsilon^{2} \sqrt{4-x_{2}} \sqrt{-x_{2}}\left(I_{0,2,0,0,0,1,2,0,0}^{A} / 2+I_{0,2,0,0,0,2,1,0,0}^{A}\right) \\
f_{4}^{A}= & \epsilon^{2} x_{1} I_{0,2,2,0,0,1,0,0,0}^{A}, \\
f_{5}^{A}= & \epsilon^{2} \sqrt{4-x_{1}} \sqrt{-x_{1}}\left(I_{0,2,2,0,0,1,0,0,0}^{A} / 2+I_{0,2,1,0,0,2,0,0,0}^{A}\right) \\
f_{6}^{A}= & \epsilon^{2} \sqrt{4-x_{1}} \sqrt{-x_{1}} I_{0,0,2,1,2,0,0,0,0}^{A}, \\
f_{7}^{A}= & \epsilon^{2} \sqrt{4-x_{2}} \sqrt{-x_{2}} I_{0,0,0,2,2,0,1,0,0}^{A}, \\
f_{8}^{A}= & \epsilon^{3}\left(x_{2}-x_{1}\right) I_{1,1,0,0,0,1,2,0,0}^{A} \\
f_{9}^{A}= & \epsilon^{2}\left(x_{2}-x_{1}\right) I_{1,1,0,0,0,1,3,0,0}^{A} \\
f_{10}^{A}= & -\epsilon^{2} \frac{\sqrt{4-x_{1}}}{4 \sqrt{-x_{1}}}\left(2 \epsilon\left(x_{2}+x_{1}\right) I_{1,1,0,0,0,1,2,0,0}^{A}-4\left(x_{2}+x_{1}\right) I_{1,1,0,0,0,1,3,0,0}^{A}\right. \\
& \left.+4 x_{1} I_{2,1,0,0,0,1,2,0,-1}^{A}+x_{2} I_{0,2,0,0,0,1,2,0,0}^{A}\right) \\
f_{11}^{A}= & \epsilon^{2} x_{3} I_{0,0,0,0,2,1,2,0,0}^{A}, \\
f_{12}^{A}= & \epsilon^{2} \sqrt{4-x_{3}} \sqrt{-x_{3}}\left(I_{0,0,0,0,2,1,2,0,0}^{A} / 2+I_{0,0,0,0,2,2,1,0,0}^{A}\right) \\
f_{13}^{A}= & \epsilon^{3}\left(x_{2}-x_{1}\right) I_{2,0,0,1,0,1,1,0,0}^{A}, \\
f_{14}^{A}= & \epsilon^{2}\left(x_{2}-x_{1}\right) I_{3,0,0,1,0,1,1,0,0}^{A}, \\
f_{15}^{A}= & \epsilon^{2} \frac{\sqrt{4-x_{2}}}{\sqrt{-x_{2}}\left(2-x_{1}\right)}\left(\epsilon \frac{2 x_{2}-x_{1}\left(x_{1}-x_{2}\right)}{2} I_{2,0,0,1,0,1,1,0,0}^{A}+x_{1}\left(x_{1}-x_{2}\right) I_{3,0,0,1,0,1,1,0,0}^{A}\right. \\
& \left.+\frac{x_{2}\left(x_{2}+x_{1}\left(x_{1}-x_{2}\right)\right)}{x_{1}-x_{2}} I_{2,0,-1,2,0,1,1,0,0}^{A}-\frac{x_{1}\left(4 x_{2}+x_{1}\left(x_{1}-x_{2}\right)\right)}{4\left(x_{1}-x_{2}\right)} I_{0,2,2,0,0,1,0,0,0}^{A}\right) \\
&
\end{aligned}
$$




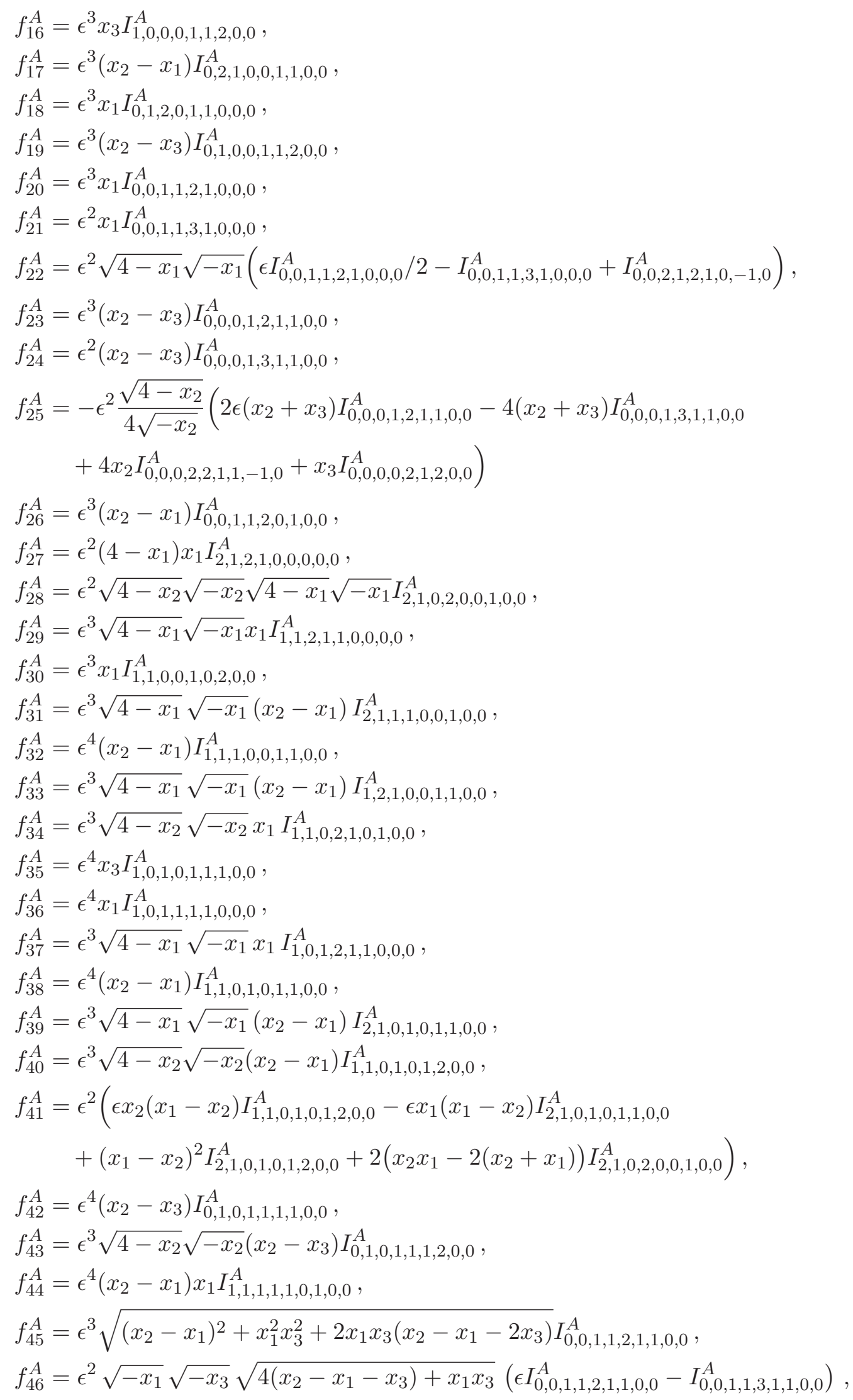




$$
\begin{aligned}
& f_{47}^{A}=\epsilon^{3}\left(x_{2}-x_{1}\right) I_{0,0,1,1,2,1,1,-1,0}^{A}, \\
& f_{48}^{A}=\epsilon^{4}\left(x_{2}-x_{1}-x_{3}\right) I_{0,1,1,0,1,1,1,0,0}^{A}, \\
& f_{49}^{A}=\epsilon^{3} \sqrt{-x_{1}} \sqrt{-x_{3}} \sqrt{x_{1} x_{3}+4\left(x_{2}-x_{1}-x_{3}\right)} I_{0,1,1,0,1,2,1,0,0}^{A}, \\
& f_{50}^{A}=\epsilon^{3}\left(x_{2}-x_{1}-x_{3}\right)\left(I_{0,1,1,0,2,1,1,0,0}^{A}+I_{0,2,1,0,1,1,1,0,0}^{A}\right) \text {, } \\
& f_{51}^{A}=\epsilon^{3}\left(x_{2}-x_{1}-x_{3}\right)\left(I_{0,1,1,0,1,1,2,0,0}^{A}+I_{0,1,2,0,1,1,1,0,0}^{A}\right), \\
& f_{52}^{A}=\epsilon^{3} \sqrt{-x_{1}} \sqrt{-\left(x_{1}+x_{1} x_{3}^{2}+2 x_{3}\left(2 x_{2}-x_{1}-2 x_{3}\right)\right)} I_{1,1,0,0,1,1,2,0,0}^{A}, \\
& f_{53}^{A}=\epsilon^{2} \sqrt{-x_{1}} \sqrt{-x_{3}} \sqrt{4\left(x_{2}-x_{1}-x_{3}\right)+x_{1} x_{3}}\left(I_{1,1,0,0,1,1,3,0,0}^{A}-\epsilon I_{1,1,0,0,1,1,2,0,0}^{A}\right) \text {, } \\
& f_{54}^{A}=\epsilon^{3} x_{1} I_{1,1,0,0,1,1,2,0,-1}^{A}, \\
& f_{55}^{A}=\epsilon^{4} \sqrt{-x_{1}} \sqrt{-x_{3}} \sqrt{4\left(x_{2}-x_{1}-x_{3}\right)+x_{1} x_{3}} I_{0,1,1,1,1,1,1,0,0}^{A}, \\
& f_{56}^{A}=-\epsilon^{4}\left(\left(2 x_{2}-2 x_{1}-x_{3}\right) I_{0,1,1,0,1,1,1,0,0}^{A}+\left(x_{1}-x_{2}\right) I_{0,1,1,1,1,1,1,-1,0}^{A}+\left(x_{1}-x_{2}\right) I_{0,1,1,1,1,1,1,0,0}^{A}\right) \text {, } \\
& f_{57}^{A}=\epsilon^{2}\left(2\left(x_{2}+x_{1}\right) I_{0,0,0,1,3,1,1,0,0}^{A}+\epsilon\left(x_{1}+x_{3}\right) I_{1,0,-1,1,1,2,1,0,0}^{A}-2 \epsilon\left(x_{2}+x_{1}\right) I_{0,0,0,1,2,1,1,0,0}^{A}\right) \text {, } \\
& f_{58}^{A}=\epsilon^{4}\left(x_{1}+x_{3}\right) I_{1,0,0,1,1,1,1,0,0}^{A}, \\
& f_{59}^{A}=\epsilon^{3} \sqrt{4-x_{2}} \sqrt{-x_{2}}\left(x_{1} I_{1,0,0,2,1,1,1,0,0}^{A}-x_{3} I_{1,0,0,1,1,1,2,0,0}^{A}\right), \\
& f_{60}^{A}=\epsilon^{3} \sqrt{-x_{1}} \sqrt{-x_{3}} \sqrt{x_{1} x_{3}-4\left(-x_{2}+x_{1}+x_{3}\right)} I_{1,0,0,1,1,2,1,0,0}^{A}, \\
& f_{61}^{A}=\epsilon^{3}\left(\left(x_{1}+x_{3}\right)-x_{2} x_{3} / 2\right)\left(I_{1,0,0,1,1,1,2,0,0}^{A}+I_{1,0,0,2,1,1,1,0,0}^{A}\right) \text {, } \\
& f_{62}^{A}=\epsilon^{4} \sqrt{-x_{1}} \sqrt{-x_{3}} \sqrt{x_{1} x_{3}-4\left(-x_{2}+x_{1}+x_{3}\right)} I_{1,0,1,1,1,1,1,0,0}^{A}, \\
& f_{63}^{A}=\epsilon^{4}\left(\left(x_{2}+x_{3}\right) I_{1,0,0,1,1,1,1,0,0}^{A}+\left(x_{1}-x_{2}\right) I_{1,0,1,1,1,1,1,-1,0}^{A}+\left(x_{1}-x_{2}\right) I_{1,0,1,1,1,1,1,0,0}^{A}\right) \text {, } \\
& f_{64}^{A}=\epsilon^{4} \sqrt{-x_{1}} \sqrt{-x_{3}} \sqrt{4\left(x_{2}-x_{1}-x_{3}\right)+x_{1} x_{3}} I_{1,1,1,0,1,1,1,0,0}^{A}, \\
& f_{65}^{A}=\epsilon^{4}\left(x_{1} I_{1,1,1,0,1,1,1,0,-1}^{A}+x_{1} I_{1,1,1,0,1,1,1,0,0}^{A}-\left(x_{2}-x_{3}\right) I_{0,1,1,0,1,1,1,0,0}^{A}\right), \\
& f_{66}^{A}=\epsilon^{4}\left(-x_{1}\right)^{3 / 2} I_{1,1,0,1,1,1,1,0,0}^{A}, \\
& f_{67}^{A}=\epsilon^{4}\left(-x_{1}\right)^{3 / 2} x_{1} I_{2,1,0,1,1,1,1,0,0}^{A}, \\
& f_{68}^{A}=\epsilon^{4}\left(-x_{1}\right)^{3 / 2} x_{1} I_{1,2,0,1,1,1,1,0,0}^{A}, \\
& f_{69}^{A}=\epsilon^{4}\left(-x_{1}\right)^{3 / 2} I_{1,1,0,1,1,1,2,-1,0}^{A}, \\
& f_{70}^{A}=\epsilon^{4} x_{1} \sqrt{-x_{3}} \sqrt{4-x_{1}} \sqrt{4\left(x_{2}-x_{1}-x_{3}\right)+x_{1} x_{3}} I_{1,1,1,1,1,1,1,0,0}^{A}, \\
& f_{71}^{A}=\epsilon^{4} \sqrt{4-x_{1}} \sqrt{-x_{1}}\left(\left(x_{2}-x_{1}\right)\left(I_{1,1,1,1,1,1,1,-1,0}^{A}+I_{1,1,1,1,1,1,1,0,0}^{A}\right)\right. \\
& \left.-x_{3} \frac{\sqrt{4\left(x_{2}-x_{1}-x_{3}\right)+x_{1} x_{3}}}{\sqrt{4 x_{2}-x_{3}-x_{1}\left(4-x_{3}\right)}} I_{1,1,1,0,1,1,1,0,0}^{A}+4 \frac{x_{1}-x_{2}}{4-x_{1}} I_{1,1,0,1,1,1,1,0,0}^{A}\right) \text {, } \\
& f_{72}^{A}=\epsilon^{4} \sqrt{-x_{1}} \sqrt{4-x_{1}}\left(x_{1}\left(I_{1,1,1,1,1,1,1,0,0}^{A}+I_{1,1,1,1,1,1,1,0,-1}^{A}\right)\right. \\
& \left.+\frac{\sqrt{4\left(x_{2}-x_{1}-x_{3}\right)+x_{1} x_{3}}}{\sqrt{4\left(x_{2}-x_{3}\right)-x_{1}\left(4-x_{3}\right)}}\left(x_{3} I_{1,0,1,1,1,1,1,0,0}^{A}+\left(x_{3}-x_{2}\right) I_{0,1,1,1,1,1,1,0,0}^{A}\right)\right) \text {, }
\end{aligned}
$$




$$
\begin{aligned}
f_{73}^{A}= & \epsilon^{4}\left(\frac { x _ { 1 } } { 2 } \left(\left(2+x_{2}-2 x_{1}\right)\left(I_{1,1,1,1,1,1,1,0,0}^{A}+I_{1,1,1,1,1,1,1,-1,0}^{A}\right)\right.\right. \\
& \left.+\left(2-x_{1}\right) I_{1,1,1,1,1,1,1,0,-1}^{A}+2 I_{1,1,1,1,1,1,1,-1,-1}^{A}\right)-2\left(x_{1}+x_{3}\right) I_{1,1,0,1,1,1,1,0,0}^{A} \\
& +\frac{x_{1} \sqrt{4\left(x_{2}-x_{1}-x_{3}\right)+x_{1} x_{3}}}{2 \sqrt{4\left(x_{2}-x_{3}\right)-x_{1}\left(4-x_{3}\right)}}\left(\left(x_{2}-x_{3}\right) I_{0,1,1,1,1,1,1,0,0}^{A}\right. \\
& \left.\left.-x_{3}\left(I_{1,0,1,1,1,1,1,0,0}^{A}+I_{1,1,1,0,1,1,1,0,0}^{A}\right)\right)\right) \\
& +\epsilon^{3} \frac{x_{1}}{4}\left(2 x_{1}\left(I_{1,0,1,2,1,1,0,0,0}^{A}-I_{1,1,2,1,1,0,0,0,0}^{A}\right)\right. \\
& \left.+\left(x_{1}-x_{2}\right)\left(I_{1,2,1,0,0,1,1,0,0}^{A}+I_{2,1,0,1,0,1,1,0,0}^{A}-2 I_{2,1,1,1,0,0,1,0,0}^{A}\right)\right) .
\end{aligned}
$$

Family B. Family B is defined by the nine propagators,

$$
\begin{array}{lll}
d_{1}^{B}=-k_{1}^{2}, & d_{2}^{B}=-\left(k_{1}+p_{12}\right)^{2}, & d_{3}^{B}=m^{2}-k_{2}^{2}, \\
d_{4}^{B}=m^{2}-\left(k_{2}+p_{12}\right)^{2}, & d_{5}^{B}=-\left(k_{1}+p_{1}\right)^{2}, & d_{6}^{B}=m^{2}-\left(k_{1}-k_{2}\right)^{2}, \\
d_{7}^{B}=m^{2}-\left(k_{2}-p_{3}\right)^{2}, & d_{8}^{B}=m^{2}-\left(k_{2}+p_{1}\right)^{2}, & d_{9}^{B}=-\left(k_{1}-p_{3}\right)^{2},
\end{array}
$$

with the extra restriction that $a_{8}$ and $a_{9}$ are non-positive. The family contains 50 master integrals. Below, we give the basis transformation between pre-canonical and canonical forms.

$$
\begin{aligned}
& f_{1}^{B}=\epsilon^{2} I_{0,0,0,0,0,2,2,0,0}^{B}, \\
& f_{2}^{B}=\epsilon^{2} x_{1} I_{1,2,0,0,0,0,2,0,0}^{B} \\
& f_{3}^{B}=\epsilon^{2} x_{1} I_{0,1,2,0,0,2,0,0,0}^{B} \\
& f_{4}^{B}=\epsilon^{2} \sqrt{4-x_{1}} \sqrt{-x_{1}}\left(I_{0,1,2,0,0,2,0,0,0}^{B} / 2+I_{0,2,2,0,0,1,0,0,0}^{B}\right), \\
& f_{5}^{B}=\epsilon^{2} x_{2} I_{0,1,0,0,0,2,2,0,0}^{B} \\
& f_{6}^{B}=\epsilon^{2} \sqrt{4-x_{2}} \sqrt{-x_{2}}\left(I_{0,1,0,0,0,2,2,0,0}^{B} / 2+I_{0,2,0,0,0,2,1,0,0}^{B}\right), \\
& f_{7}^{B}=\epsilon^{2} \sqrt{4-x_{1}} \sqrt{-x_{1}} I_{0,0,1,2,0,2,0,0,0}^{B}, \\
& f_{8}^{B}=\epsilon^{2} \sqrt{4-x_{2}} \sqrt{-x_{2}} I_{0,0,0,2,0,2,1,0,0}^{B}, \\
& f_{9}^{B}=\epsilon^{2} x_{3} I_{0,0,0,0,1,2,2,0,0}^{B} \\
& f_{10}^{B}=\epsilon^{2} \sqrt{4-x_{3}} \sqrt{-x_{3}}\left(I_{0,0,0,0,1,2,2,0,0}^{B} / 2+I_{0,0,0,0,2,2,1,0,0}^{B}\right), \\
& f_{11}^{B}=\epsilon^{2} \sqrt{4-x_{1}} \sqrt{-x_{1}} x_{1} I_{1,2,1,2,0,0,0,0,0}^{B}, \\
& f_{12}^{B}=\epsilon^{2} \sqrt{4-x_{2}} \sqrt{-x_{2}} x_{1} I_{1,2,0,2,0,0,1,0,0}^{B}, \\
& f_{13}^{B}=\epsilon^{3}\left(x_{2}-x_{1}\right) I_{1,1,0,0,0,2,1,0,0}^{B}, \\
& f_{14}^{B}=\epsilon^{2} \frac{\sqrt{4+x_{1}-x_{2}}}{\sqrt{x_{1}-x_{2}}}\left(x_{1} I_{1,2,0,0,0,2,1,0,-1}^{B}-x_{2} I_{0,2,0,0,0,2,1,0,0}^{B}-\epsilon\left(x_{1}-x_{2}\right) I_{1,1,0,0,0,2,1,0,0}^{B}\right), \\
& f_{15}^{B}=\epsilon^{3}\left(x_{2}-x_{1}\right) I_{1,0,0,1,0,2,1,0,0}^{B}, \\
& f_{16}^{B}=\epsilon^{2}\left(x_{2}-x_{1}\right) I_{1,0,0,1,0,3,1,0,0}^{B},
\end{aligned}
$$




$$
\begin{aligned}
& f_{17}^{B}=\epsilon^{2} \frac{\sqrt{4-x_{2}} \sqrt{-x_{2}}}{4\left(x_{2}-2 x_{1}\right)}\left(6 \epsilon\left(x_{1}-x_{2}\right) I_{1,0,0,1,0,2,1,0,0}^{B}-4\left(x_{1}-x_{2}\right) I_{1,0,0,1,0,3,1,0,0}^{B}\right. \\
& \left.+4\left(x_{2}+x_{1}\left(x_{1}-x_{2}\right)\right) I_{1,0,0,2,0,2,1,0,0}^{B}-3 x_{1} I_{0,1,2,0,0,2,0,0,0}^{B}\right), \\
& f_{18}^{B}=\epsilon^{3}\left(x_{2}-x_{1}\right) I_{0,1,1,0,0,2,1,0,0}^{B}, \\
& f_{19}^{B}=\epsilon^{3} x_{1} I_{0,0,1,1,1,2,0,0,0}^{B} \text {, } \\
& f_{20}^{B}=\epsilon^{2} x_{1} I_{0,0,1,1,1,3,0,0,0}^{B} \text {, } \\
& f_{21}^{B}=\epsilon^{2} \sqrt{4-x_{1}} \sqrt{-x_{1}}\left(I_{0,0,1,2,1,2,0,-1,0}^{B}-\epsilon I_{0,0,1,1,1,2,0,0,0}^{B}\right), \\
& f_{22}^{B}=\epsilon^{3}\left(x_{2}-x_{1}\right) I_{0,0,1,1,0,2,1,0,0}^{B}, \\
& f_{23}^{B}=\epsilon^{3} x_{3} I_{0,0,1,0,1,2,1,0,0}^{B}, \\
& f_{24}^{B}=\epsilon^{3}\left(x_{2}-x_{3}\right) I_{0,0,0,1,1,2,1,0,0}^{B}, \\
& f_{25}^{B}=\epsilon^{2}\left(x_{2}-x_{3}\right) I_{0,0,0,1,1,3,1,0,0}^{B}, \\
& f_{26}^{B}=\epsilon^{2} \frac{\sqrt{4-x_{2}}}{\sqrt{-x_{2}}\left(x_{3}\left(2 x_{2}-x_{3}\right)-2\left(x_{2}+x_{3}\right)\right)}\left(x_{2}\left(x_{2}-x_{3}\left(x_{2}-x_{3}\right)\right) I_{0,0,0,2,1,2,1,-1,0}^{B}\right. \\
& +x_{3}^{2}\left(x_{2}-x_{3}\right) I_{0,0,0,1,1,3,1,0,0}^{B}+x_{3}\left(4 x_{2}-x_{3}\left(4 x_{2}-x_{3}\right)\right) I_{0,0,0,0,1,2,2,0,0}^{B} / 4 \\
& \left.-\epsilon\left(2 x_{2}\left(x_{2}+x_{3}\right)-x_{3}\left(2 x_{2}^{2}-3 x_{2} x_{3}+x_{3}^{2}\right)\right) I_{0,0,0,1,1,2,1,0,0}^{B} / 2\right), \\
& f_{27}^{B}=\epsilon^{3}(1-2 \epsilon) x_{1} I_{1,1,1,1,0,1,0,0,0}^{B}, \\
& f_{28}^{B}=\epsilon^{3}\left(x_{2}-x_{1}\right) x_{1} I_{1,2,1,1,0,0,1,0,0}^{B}, \\
& f_{29}^{B}=\epsilon^{4}\left(x_{2}-x_{1}\right) I_{1,1,1,0,0,1,1,0,0}^{B}, \\
& f_{30}^{B}=\epsilon^{4}\left(x_{2}-x_{1}\right) I_{1,1,0,1,0,1,1,0,0}^{B}, \\
& f_{31}^{B}=\epsilon^{2} x_{1} I_{1,1,0,1,0,2,1,0,0}^{B}+\epsilon^{3}\left(4-x_{2}\right)\left(x_{2}+x_{1}\right) I_{1,1,0,1,0,1,2,0,0}^{B} / 2 \\
& -2 \epsilon^{4} x_{2} I_{1,1,0,1,0,1,1,0,0}^{B}+\frac{\epsilon^{2}}{2\left(x_{2}-x_{1}\right)}\left(\left(x_{2}\left(x_{2}-x_{1}\right)-4\left(x_{2}+x_{1}\right)\right) I_{0,2,0,0,0,2,1,0,0}^{B}\right. \\
& \left.+2 x_{1}\left(4-x_{2}+x_{1}\right) I_{1,2,0,0,0,2,1,0,-1}^{B}\right)+\epsilon^{3}\left(4-3 x_{2}+x_{1}\right) I_{1,1,0,0,0,2,1,0,0}^{B} \\
& +\frac{\epsilon^{2}}{4\left(x_{2}-2 x_{1}\right)}\left(4\left(4 x_{2}+x_{2}\left(x_{2}-x_{1}\right) x_{1}-\left(x_{2}^{2}+4 x_{2} x_{1}-4 x_{1}^{2}\right)\right) I_{1,0,0,2,0,2,1,0,0}^{B}\right. \\
& +\left(4-x_{2}\right)\left(x_{2}-x_{1}\right) I_{1,0,0,1,0,3,1,0,0}^{B}-3\left(4-x_{2}\right) x_{1} I_{0,1,2,0,0,2,0,0,0}^{B} \\
& \left.+2 \epsilon\left(x_{2}\left(5 x_{2}-7 x_{1}\right)-12\left(x_{2}-x_{1}\right)\right) I_{1,0,0,1,0,2,1,0,0}^{B}\right) \\
& +\epsilon^{2}\left(-x_{2} / 4\right)\left(I_{0,1,0,0,0,2,2,0,0}^{B}-2 I_{0,0,0,2,0,2,1,0,0}^{B}+4 x_{1} I_{1,2,0,2,0,0,1,0,0}^{B}\right), \\
& f_{32}^{B}=\epsilon^{3} \sqrt{4-x_{2}} \sqrt{-x_{2}}\left(x_{2}-x_{1}\right) I_{1,1,0,1,0,1,2,0,0}^{B}, \\
& f_{33}^{B}=\epsilon^{3} x_{1} \sqrt{4-x_{3}} \sqrt{-x_{3}} I_{1,1,0,0,1,2,1,0,0}^{B}, \\
& f_{34}^{B}=\epsilon^{3} x_{1}\left(I_{1,1,0,0,1,2,1,0,-1}^{B}+x_{3} I_{1,1,0,0,1,2,1,0,0}^{B}\right), \\
& f_{35}^{B}=\epsilon^{4}\left(x_{1}+x_{3}\right) I_{1,0,0,1,1,1,1,0,0}^{B}, \\
& f_{36}^{B}=\epsilon^{3} \sqrt{-x_{1}} \sqrt{-x_{3}} \sqrt{4\left(x_{2}-x_{1}-x_{3}\right)+x_{1} x_{3}} I_{1,0,0,1,1,2,1,0,0}^{B},
\end{aligned}
$$




$$
\begin{aligned}
& f_{37}^{B}=\epsilon^{2} \frac{2\left(x_{1}+x_{3}\right)-x_{2} x_{3}}{4 x_{2} x_{3}\left(2\left(x_{2}+x_{3}\right)-x_{3}\left(2 x_{2}-x_{3}\right)\right)}\left(x_{3}\left(4 x_{2}-x_{3}\left(4 x_{2}-x_{3}\right)\right) I_{0,0,0,0,1,2,2,0,0}^{B}\right. \\
& +2 \epsilon\left(x_{3}\left(2 x_{2}^{2}-3 x_{2} x_{3}+x_{3}^{2}\right)-2 x_{2}\left(x_{2}+x_{3}\right)\right) I_{0,0,0,1,1,2,1,0,0}^{B} \\
& \left.+4\left(x_{2}-x_{3}\right) x_{3}^{2} I_{0,0,0,1,1,3,1,0,0}^{B}+4 x_{2}\left(x_{2}-x_{3}\left(x_{2}-x_{3}\right)\right) I_{0,0,0,2,1,2,1,-1,0}^{B}\right) \\
& +\epsilon^{2} \frac{2\left(x_{1}+x_{3}\right)-x_{2} x_{3}}{4 x_{3}\left(x_{2}-2 x_{1}\right)}\left(3 x_{1} I_{0,1,2,0,0,2,0,0,0}^{B}+6 \epsilon\left(x_{2}-x_{1}\right) I_{1,0,0,1,0,2,1,0,0}^{B}\right. \\
& \left.-4\left(x_{2}-x_{1}\right) I_{1,0,0,1,0,3,1,0,0}^{B}-4\left(x_{2}-x_{1}\left(x_{2}-x_{1}\right)\right) I_{1,0,0,2,0,2,1,0,0}^{B}\right) \\
& +\epsilon^{3}\left(\left(2\left(x_{1}+x_{3}\right)-x_{1} x_{3}\right) I_{1,0,0,1,1,2,1,0,0}^{B} / 2+\left(\left(x_{1}+x_{3}\right)^{2}-x_{2} x_{1} x_{3}\right) I_{1,0,0,2,1,1,1,0,0}^{B} / x_{3}\right) \\
& f_{38}^{B}=\epsilon^{3} \sqrt{\left(x_{2}-x_{1}\right)^{2}+x_{1}^{2} x_{3}^{2}+2 x_{1} x_{3}\left(x_{2}-x_{1}-2 x_{3}\right)} I_{0,0,1,1,1,2,1,0,0}^{B}, \\
& f_{39}^{B}=\epsilon^{2} \sqrt{-x_{1}} \sqrt{-x_{3}} \sqrt{4\left(x_{2}-x_{1}-x_{3}\right)+x_{1} x_{3}}\left(I_{0,0,1,1,1,3,1,0,0}^{B}-\epsilon I_{0,0,1,1,1,2,1,0,0}^{B}\right) \\
& f_{40}^{B}=\epsilon^{3}\left(x_{2}-x_{1}\right)\left(I_{0,0,1,1,1,2,1,-1,0}^{B}-I_{0,0,1,1,1,2,1,0,0}^{B}\right), \\
& \left.f_{41}^{B}=\epsilon^{4} \sqrt{4-x_{1}} \sqrt{-x_{1}}\left(x_{2}-x_{1}\right) I_{1,1,1,1,0,1,1,0,0}^{B}\right), \\
& f_{42}^{B}=\epsilon^{4}\left(x_{2}-x_{1}-x_{3}\right) I_{0,1,1,0,1,1,1,0,0}^{B}, \\
& f_{43}^{B}=\epsilon^{3} \sqrt{x_{1} x_{3}\left(4\left(x_{2}-x_{1}-x_{3}\right)+x_{1} x_{3}\right)} I_{0,1,1,0,1,2,1,0,0}^{B}, \\
& f_{44}^{B}=\epsilon^{4} x_{1} x_{3} I_{1,1,1,0,1,1,1,0,0}^{B}, \\
& f_{45}^{B}=\epsilon^{4} x_{1}\left(x_{2}-x_{3}\right) I_{1,1,0,1,1,1,1,0,0}^{B}, \\
& f_{46}^{B}=\epsilon^{2} x_{1} \sqrt{4-x_{2}} \sqrt{-x_{2}}\left(2 I_{1,1,0,0,1,2,1,0,0}^{B}-I_{1,0,0,1,1,2,1,0,0}^{B}+\left(x_{2}-x_{3}\right) I_{1,1,0,1,1,1,2,0,0}^{B}\right) \text {, } \\
& f_{47}^{B}=\epsilon^{4} x_{1} \sqrt{-x_{1}} \sqrt{-x_{3}} \sqrt{4\left(x_{2}-x_{1}-x_{3}\right)+x_{1} x_{3}} I_{1,1,1,1,1,1,1,0,0}^{B}, \\
& f_{48}^{B}=\epsilon^{4}\left(x_{2}-x_{1}\right) x_{1} I_{1,1,1,1,1,1,1,-1,0}^{B}, \\
& f_{49}^{B}=\epsilon^{2} \sqrt{-x_{1}} \sqrt{4-x_{1}}\left(\epsilon^{2} x_{1} x_{3} I_{1,1,1,1,1,1,1,0,0}^{B}+\epsilon^{2} x_{1} I_{1,1,1,1,1,1,1,0,-1}^{B}+\epsilon x_{3} I_{1,0,0,1,1,2,1,0,0}^{B} / 2\right. \\
& \left.-\epsilon\left(x_{2}-x_{3}\right) I_{0,1,1,0,1,2,1,0,0}^{B} / 2+\left(x_{2}-2 x_{3}\right)\left(\epsilon I_{0,0,1,1,1,2,1,0,0}^{B}-I_{0,0,1,1,1,3,1,0,0}^{B}\right)\right), \\
& f_{50}^{B}=2 \epsilon^{4} x_{1}\left(2 I_{1,1,1,1,1,1,1,-1,-1}^{B}+2\left(x_{2}-x_{1}\right) I_{1,1,1,1,1,1,1,-1,0}^{B}-x_{1} I_{1,1,1,1,1,1,1,0,-1}^{B}\right. \\
& \left.-x_{1} x_{3} I_{1,1,1,1,1,1,1,0,0}^{B}\right)+\epsilon^{2} \frac{x_{2}}{x_{2}-x_{1}}\left(x_{2} I_{0,1,0,0,0,2,2,0,0}^{B}-x_{1} I_{0,1,2,0,0,2,0,0,0}^{B}\right) \\
& -2 \epsilon^{3} x_{2}\left(I_{0,0,1,1,0,2,1,0,0}^{B}-2 I_{0,0,1,1,1,2,1,-1,0}^{B}+I_{0,1,1,0,0,2,1,0,0}^{B}\right) \\
& 2 \epsilon^{2} x_{1}\left(x_{2}-2 x_{3}\right) I_{0,0,1,1,1,3,1,0,0}^{B}-2 \epsilon^{3}\left(2 x_{2}+x_{1}\left(x_{2}-2 x_{3}\right)\right) I_{0,0,1,1,1,2,1,0,0}^{B} \\
& +\epsilon^{3} x_{1}\left(\left(x_{2}-x_{3}\right) I_{0,1,1,0,1,2,1,0,0}^{B}-x_{3} I_{1,0,0,1,1,2,1,0,0}^{B}-4 x_{3} I_{1,2,1,1,0,0,1,0,0}^{B}\right) \\
& -4 \epsilon^{4} x_{1}\left(I_{0,1,1,0,1,1,1,0,0}^{B}+I_{1,0,0,1,1,1,1,0,0}^{B}+I_{1,1,1,1,0,1,0,0,0}^{B}\right) \\
& +4 \epsilon^{4} x_{2} I_{1,1,0,1,0,1,1,0,0}^{B}-2 \epsilon^{4}\left(x_{2}-x_{1}\right) x_{1} I_{1,1,1,1,0,1,1,0,0}^{B} .
\end{aligned}
$$


Family C. Family $\mathrm{C}$ is defined by the nine propagators,

$$
\begin{array}{lll}
d_{1}^{C}=-k_{1}^{2}, & d_{2}^{C}=-\left(k_{1}+p_{12}\right)^{2}, & d_{3}^{C}=m^{2}-\left(k_{2}+p_{12}\right)^{2}, \\
d_{4}^{C}=-\left(k_{1}+p_{1}\right)^{2}, & d_{5}^{C}=m^{2}-\left(k_{1}-k_{2}\right)^{2}, & d_{6}^{C}=m^{2}-\left(k_{2}-p_{3}\right)^{2}, \\
d_{7}^{C}=-\left(k_{1}-p_{3}\right)^{2}, & d_{8}^{C}=m^{2}-k_{2}^{2}, & d_{9}^{C}=m^{2}-\left(k_{2}+p_{1}\right)^{2},
\end{array}
$$

with the extra restriction that $a_{8}$ and $a_{9}$ are non-positive. The family contains 45 master integrals. Below, we give the basis transformation between pre-canonical and canonical forms.

$$
\begin{aligned}
& f_{1}^{C}=\epsilon^{2} I_{0,0,0,0,2,2,0,0,0}^{C}, \\
& f_{2}^{C}=\epsilon^{2} x_{3} I_{0,0,0,1,0,2,2,0,0}^{C} \text {, } \\
& f_{3}^{C}=\epsilon^{2} x_{3} I_{0,0,0,1,2,2,0,0,0}^{C}, \\
& f_{4}^{C}=\epsilon^{2} \sqrt{4-x_{3}} \sqrt{-x_{3}}\left(I_{0,0,0,2,1,2,0,0,0}^{C}+I_{0,0,0,1,2,2,0,0,0}^{C} / 2\right), \\
& f_{5}^{C}=\epsilon^{2} x_{2} I_{0,0,2,0,2,0,1,0,0}^{C}, \\
& f_{6}^{C}=\epsilon^{2} \sqrt{4-x_{2}} \sqrt{-x_{2}}\left(I_{0,0,1,0,2,0,2,0,0}^{C}+I_{0,0,2,0,2,0,1,0,0}^{C} / 2\right), \\
& f_{7}^{C}=\epsilon^{2} \sqrt{4-x_{2}} \sqrt{-x_{2}} I_{0,0,1,0,2,2,0,0,0}^{C}, \\
& f_{8}^{C}=\epsilon^{2} x_{2} I_{0,1,0,0,0,2,2,0,0}^{C} \text {, } \\
& f_{9}^{C}=\epsilon^{2} x_{1} I_{1,0,2,0,2,0,0,0,0}^{C}, \\
& f_{10}^{C}=\epsilon^{2} \sqrt{4-x_{1}} \sqrt{-x_{1}}\left(I_{2,0,1,0,2,0,0,0,0}^{C}+I_{1,0,2,0,2,0,0,0,0}^{C} / 2\right), \\
& f_{11}^{C}=\epsilon^{2} x_{1} I_{1,2,0,0,0,2,0,0,0}^{C}, \\
& f_{12}^{C}=\epsilon^{2} x_{3} \sqrt{4-x_{2}} \sqrt{-x_{2}} I_{0,0,1,1,0,2,2,0,0}^{C}, \\
& f_{13}^{C}=\epsilon^{3}\left(x_{2}-x_{3}\right) I_{0,0,1,1,2,0,1,0,0}^{C}, \\
& f_{14}^{C}=\epsilon^{2} \frac{\sqrt{4-x_{2}+x_{3}}}{\sqrt{x_{3}-x_{2}}}\left(x_{3} I_{0,-1,1,1,2,0,2,0,0}^{C}-x_{2} I_{0,0,1,0,2,0,2,0,0}^{C}-\epsilon\left(x_{3}-x_{2}\right) I_{0,0,1,1,2,0,1,0,0}^{C}\right), \\
& f_{15}^{C}=\epsilon^{3}\left(x_{2}-x_{3}\right) I_{0,0,1,1,2,1,0,0,0}^{C}, \\
& f_{16}^{C}=\epsilon^{2}\left(x_{2}-x_{3}\right) I_{0,0,1,1,3,1,0,0,0}^{C}, \\
& f_{17}^{C}=\epsilon^{2} \frac{\sqrt{4-x_{2}} \sqrt{-x_{2}}}{4\left(x_{2}-2 x_{3}\right)}\left(4\left(x_{2}-x_{3}\right) I_{0,0,1,1,3,1,0,0,0}^{C}-6 \epsilon\left(x_{2}-x_{3}\right) I_{0,0,1,1,2,1,0,0,0}^{C}\right. \\
& \left.+4\left(x_{2}-x_{3}\left(x_{2}-x_{3}\right)\right) I_{0,0,1,1,2,2,0,0,0}^{C}-3 x_{3} I_{0,0,0,1,2,2,0,0,0}^{C}\right), \\
& f_{18}^{C}=\epsilon^{2} x_{2} \sqrt{4-x_{2}} \sqrt{-x_{2}} I_{0,1,1,0,0,2,2,0,0}^{C}, \\
& f_{19}^{C}=\epsilon^{3}\left(x_{2}-x_{1}\right) I_{1,0,1,0,2,1,0,0,0}^{C}, \\
& f_{20}^{C}=\epsilon^{2}\left(x_{2}-x_{1}\right) I_{1,0,1,0,3,1,0,0,0}^{C}, \\
& f_{21}^{C}=\epsilon^{2} \frac{\sqrt{4-x_{2}} \sqrt{-x_{2}}}{4\left(x_{2}-2 x_{1}\right)}\left(4\left(x_{2}-x_{1}\right) I_{1,0,1,0,3,1,0,0,0}^{C}-6 \epsilon\left(x_{2}-x_{1}\right) I_{1,0,1,0,2,1,0,0,0}^{C}\right. \\
& \left.+4\left(x_{2}-x_{1}\left(x_{2}-x_{1}\right)\right) I_{1,0,2,0,2,1,0,0,0}^{C}-3 x_{1} I_{1,0,2,0,2,0,0,0,0}^{C}\right), \\
& f_{22}^{C}=\epsilon^{3}\left(x_{2}-x_{1}\right) I_{1,1,0,0,2,1,0,0,0}^{C}, \\
& f_{23}^{C}=\epsilon^{2} \frac{\sqrt{4-x_{2}+x_{1}}}{\sqrt{x_{1}-x_{2}}}\left(x_{1} I_{1,2,0,0,2,1,-1,0,0}^{C}-x_{2} I_{0,2,0,0,2,1,0,0,0}^{C}-\epsilon\left(x_{1}-x_{2}\right) I_{1,1,0,0,2,1,0,0,0}^{C}\right),
\end{aligned}
$$




$$
\begin{aligned}
& f_{24}^{C}=\epsilon^{2} x_{1} \sqrt{4-x_{2}} \sqrt{-x_{2}} I_{1,2,1,0,0,2,0,0,0}^{C}, \\
& f_{25}^{C}=\epsilon^{4}\left(x_{2}-x_{3}\right) I_{0,0,1,1,1,1,1,0,0}^{C} \text {, } \\
& f_{26}^{C}=\epsilon^{2} x_{3} I_{0,0,1,1,2,1,1,0,0}^{C}+\epsilon^{3}\left(4-x_{2}\right)\left(x_{2}+x_{3}\right) I_{0,0,2,1,1,1,1,0,0}^{C} / 2 \\
& -2 \epsilon^{4} x_{2} I_{0,0,1,1,1,1,1,0,0}^{C}+\frac{\epsilon^{2}}{2\left(x_{2}-x_{3}\right)}\left(\left(x_{2}\left(x_{2}-x_{3}\right)-4\left(x_{2}+x_{3}\right)\right) I_{0,0,1,0,2,0,2,0,0}^{C}\right. \\
& \left.+2 x_{3}\left(4-x_{2}+x_{3}\right) I_{0,-1,1,1,2,0,2,0,0}^{C}\right)+\epsilon^{3}\left(4-3 x_{2}+x_{3}\right) I_{0,0,1,1,2,0,1,0,0}^{C} \\
& +\frac{\epsilon^{2}}{4\left(x_{2}-2 x_{3}\right)}\left(2 \epsilon\left(x_{2}\left(5 x_{2}-7 x_{3}\right)-12\left(x_{2}-x_{3}\right)\right) I_{0,0,1,1,2,1,0,0,0}^{C}\right. \\
& +4\left(4 x_{2}+x_{2}\left(x_{2}-x_{3}\right) x_{3}-\left(x_{2}^{2}+4 x_{2} x_{3}-4 x_{3}^{2}\right)\right) I_{0,0,1,1,2,2,0,0,0}^{C} \\
& \left.+4\left(4-x_{2}\right)\left(x_{2}-x_{3}\right) I_{0,0,1,1,3,1,0,0,0}^{C}-3\left(4-x_{2}\right) x_{3} I_{0,0,0,1,2,2,0,0,0}^{C}\right) \\
& +\epsilon^{2}\left(4-x_{2}\right)\left(x_{3} I_{0,0,1,1,0,2,2,0,0}^{C}-I_{0,0,1,0,2,2,0,0,0}^{C} / 2+I_{0,0,2,0,2,0,1,0,0}^{C} / 4\right), \\
& f_{27}^{C}=\epsilon^{3} \sqrt{4-x_{2}} \sqrt{-x_{2}}\left(x_{2}-x_{3}\right) I_{0,0,2,1,1,1,1,0,0}^{C}, \\
& f_{28}^{C}=(1-2 \epsilon) \epsilon^{3} x_{2} I_{0,1,1,0,1,1,1,0,0}^{C}, \\
& f_{29}^{C}=\epsilon^{3} \sqrt{4-x_{1}} \sqrt{-x_{1}} x_{3} I_{1,0,1,1,2,0,1,0,0}^{C}, \\
& f_{30}^{C}=\epsilon^{3} x_{3}\left(I_{1,-1,1,1,2,0,1,0,0}^{C}+x_{1} I_{1,0,1,1,2,0,1,0,0}^{C}\right) \text {, } \\
& f_{31}^{C}=\epsilon^{4}\left(x_{1}+x_{3}\right) I_{1,0,1,1,1,1,0,0,0}^{C}, \\
& f_{32}^{C}=\epsilon^{3} \sqrt{-x_{1}} \sqrt{-x_{3}} \sqrt{4\left(x_{2}-x_{1}-x_{3}\right)+x_{1} x_{3}} I_{1,0,1,1,2,1,0,0,0}^{C}, \\
& f_{33}^{C}=\epsilon^{2} \frac{2\left(x_{1}+x_{3}\right)-x_{2} x_{3}}{4 x_{2} x_{3}\left(2\left(x_{2}+x_{3}\right)-x_{3}\left(2 x_{2}-x_{3}\right)\right)}\left(x_{3}\left(4 x_{2}-x_{3}\left(4 x_{2}-x_{3}\right)\right) I_{0,0,0,1,2,2,0,0,0}^{C}\right. \\
& -2 \epsilon\left(2 x_{2}\left(x_{2}+x_{3}\right)-x_{3}\left(2 x_{2}^{2}-3 x_{2} x_{3}+x_{3}^{2}\right)\right) I_{0,0,1,1,2,1,0,0,0}^{C} \\
& \left.+4\left(x_{2}-x_{3}\right) x_{3}^{2} I_{0,0,1,1,3,1,0,0,0}^{C}+4 x_{2}\left(x_{2}-x_{3}\left(x_{2}-x_{3}\right)\right) I_{0,0,2,1,2,1,0,0,-1}^{C}\right) \\
& +\epsilon^{2} \frac{2\left(x_{1}+x_{3}\right)-x_{2} x_{3}}{4\left(x_{2}-2 x_{1}\right) x_{3}}\left(3 x_{1} I_{1,0,2,0,2,0,0,0,0}^{C}-4\left(x_{2}-x_{1}\right) I_{1,0,1,0,3,1,0,0,0}^{C}\right. \\
& \left.+6 \epsilon\left(x_{2}-x_{1}\right) I_{1,0,1,0,2,1,0,0,0}^{C}-4\left(x_{2}+x_{1}\left(-x_{2}+x_{1}\right)\right) I_{1,0,2,0,2,1,0,0,0}^{C}\right) \\
& \left.+\epsilon^{3}\left(\left(2\left(x_{1}+x_{3}\right)-x_{1} x_{3}\right) I_{1,0,1,1,2,1,0,0,0}^{C} / 2+\left(\left(x_{1}+x_{3}\right)^{2}-x_{2} x_{1} x_{3}\right) I_{1,0,2,1,1,1,0,0,0}^{C}\right) / x_{3}\right), \\
& f_{34}^{C}=\epsilon^{3} x_{1} x_{3} I_{1,1,0,1,0,2,1,0,0}^{C}, \\
& f_{35}^{C}=\epsilon^{3} x_{1} \sqrt{4-x_{3}} \sqrt{-x_{3}} I_{1,1,0,1,2,1,0,0,0}^{C}, \\
& f_{36}^{C}=\epsilon^{3} x_{1}\left(I_{1,1,0,1,2,1,-1,0,0}^{C}+x_{3} I_{1,1,0,1,2,1,0,0,0}^{C}\right), \\
& f_{37}^{C}=\epsilon^{4}\left(x_{2}-x_{1}\right) I_{1,1,1,0,1,1,0,0,0}^{C}, \\
& f_{38}^{C}=\epsilon^{2} x_{1} I_{1,1,1,0,2,1,0,0,0}^{C}+\epsilon^{3}\left(4-x_{2}\right)\left(x_{2}+x_{1}\right) I_{1,1,1,0,1,2,0,0,0}^{C} / 2 \\
& -2 \epsilon^{4} x_{2} I_{1,1,1,0,1,1,0,0,0}^{C}+\frac{\epsilon^{2}}{2\left(x_{2}-x_{1}\right)}\left(\left(x_{2}\left(x_{2}-x_{1}\right)-4\left(x_{2}+x_{1}\right)\right) I_{0,2,0,0,2,1,0,0,0}^{C}\right. \\
& \left.+2 x_{1}\left(4-x_{2}+x_{1}\right) I_{1,2,0,0,2,1,-1,0,0}^{C}\right)+\epsilon^{3}\left(4-3 x_{2}+x_{1}\right) I_{1,1,0,0,2,1,0,0,0}^{C} \\
& +\frac{\epsilon^{2}}{4\left(x_{2}-2 x_{1}\right)}\left(2 \epsilon\left(x_{2}\left(5 x_{2}-7 x_{1}\right)-12\left(x_{2}-x_{1}\right)\right) I_{1,0,1,0,2,1,0,0,0}^{C}\right.
\end{aligned}
$$




$$
\begin{aligned}
& +4\left(4 x_{2}+x_{2}\left(x_{2}-x_{1}\right) x_{1}-\left(x_{2}^{2}+4 x_{2} x_{1}-4 x_{1}^{2}\right)\right) I_{1,0,2,0,2,1,0,0,0}^{C} \\
& \left.4\left(4-x_{2}\right)\left(x_{2}-x_{1}\right) I_{1,0,1,0,3,1,0,0,0}^{C}-3\left(4-x_{2}\right) x_{1} I_{1,0,2,0,2,0,0,0,0}^{C}\right) \\
& +\epsilon^{2}\left(4-x_{2}\right)\left(I_{0,1,0,0,2,2,0,0,0}^{C} / 4-I_{0,0,2,0,2,1,0,0,0}^{C} / 2+x_{1} I_{1,2,2,0,0,1,0,0,0}^{C}\right) \\
f_{39}^{C}= & \epsilon^{3} \sqrt{4-x_{2}} \sqrt{-x_{2}}\left(x_{2}-x_{1}\right) I_{1,1,1,0,1,2,0,0,0}^{C}, \\
f_{40}^{C}= & \epsilon^{4}\left(x_{2}-x_{1}\right) x_{3} I_{1,0,1,1,1,1,1,0,0}^{C} \\
f_{41}^{C}= & \epsilon^{3} \sqrt{4-x_{2}} \sqrt{-x_{2}} x_{3}\left(2 I_{1,0,1,1,2,0,1,0,0}^{C}-I_{1,0,1,1,2,1,0,0,0}^{C}+\left(x_{2}-x_{1}\right) I_{1,0,2,1,1,1,1,0,0}^{C}\right), \\
f_{42}^{C}= & \epsilon^{3} \sqrt{4-x_{2}} \sqrt{-x_{2}} x_{1} x_{3} I_{1,1,1,1,0,2,1,0,0}^{C} \\
f_{43}^{C}= & \epsilon^{4} x_{1}\left(x_{2}-x_{3}\right) I_{1,1,1,1,1,1,0,0,0}^{C}, \\
f_{44}^{C}= & \epsilon^{3} \sqrt{4-x_{2}} \sqrt{-x_{2}} x_{1}\left(2 I_{1,1,0,1,2,1,0,0,0}^{C}-I_{1,0,1,1,2,1,0,0,0}^{C}+\left(x_{2}-x_{3}\right) I_{1,1,1,1,1,2,0,0,0}^{C}\right), \\
f_{45}^{C}= & \epsilon^{4} x_{2}\left(x_{3} I_{1,0,1,1,1,1,1,0,0}^{C}+x_{1} I_{1,1,1,1,1,1,0,0,0}^{C}+x_{1} x_{3} I_{1,1,1,1,1,1,1,0,0}^{C}\right) .
\end{aligned}
$$

Family D. Family D is defined by the nine propagators,

$$
\begin{array}{lll}
d_{1}^{D}=m^{2}-k_{1}^{2}, & d_{2}^{D}=m^{2}-\left(k_{1}+p_{12}\right)^{2}, & d_{3}^{D}=m^{2}-k_{2}^{2}, \\
d_{4}^{D}=m^{2}-\left(k_{2}+p_{12}\right)^{2}, & d_{5}^{D}=m^{2}-\left(k_{1}+p_{1}\right)^{2}, & d_{6}^{D}=-\left(k_{1}-k_{2}\right)^{2}, \\
d_{7}^{D}=m^{2}-\left(k_{2}-p_{3}\right)^{2}, & d_{8}^{D}=m^{2}-\left(k_{2}+p_{1}\right)^{2}, & d_{9}^{D}=m^{2}-\left(k_{1}-p_{3}\right)^{2},
\end{array}
$$

with the extra restriction that $a_{1}, a_{5}$, and $a_{6}$ are non-positive. The family contains 17 master integrals. Below, we give the basis transformation between pre-canonical and canonical forms.

$$
\begin{aligned}
& f_{1}^{D}=\epsilon^{2} I_{0,0,0,0,0,0,2,0,2}^{D} \\
& f_{2}^{D}=-\epsilon^{2} \sqrt{4-x_{3}} \sqrt{-x_{3}} I_{0,0,0,0,0,0,1,2,2}^{D} \\
& f_{3}^{D}=-\epsilon^{2} \sqrt{4-x_{1}} \sqrt{-x_{1}} I_{0,0,2,1,0,0,0,0,2}^{D} \\
& f_{4}^{D}=-\epsilon^{2} \sqrt{4-x_{2}} \sqrt{-x_{2}} I_{0,1,2,0,0,0,0,0,2}^{D} \\
& f_{5}^{D}=\epsilon^{3}\left(x_{3}-x_{2}\right) I_{0,0,0,1,0,0,1,1,2}^{D} \\
& f_{6}^{D}=\epsilon^{3} x_{3} I_{0,0,1,0,0,0,1,1,2}^{D} \\
& f_{7}^{D}=\epsilon^{3} x_{1} I_{0,0,1,1,0,0,0,1,2}^{D} \\
& f_{8}^{D}=\epsilon^{3}\left(x_{1}-x_{2}\right) I_{0,0,1,1,0,0,1,0,2}^{D} \\
& f_{9}^{D}=\epsilon^{2} \sqrt{4-x_{2}} \sqrt{-x_{2}} \sqrt{4-x_{3}} \sqrt{-x_{3}} I_{0,1,0,0,0,0,1,2,2}^{D} \\
& f_{10}^{D}=-\epsilon^{2} x_{2}\left(4-x_{2}\right) I_{0,1,0,1,0,0,2,0,2}^{D} \\
& f_{11}^{D}=\epsilon^{2} \sqrt{4-x_{2}} \sqrt{-x_{2}} \sqrt{4-x_{1}} \sqrt{-x_{1}} I_{0,1,1,2,0,0,0,0,2}^{D} \\
& \left.f_{12}^{D}=-\epsilon^{3} \sqrt{-x_{1}} \sqrt{-x_{3}} \sqrt{4\left(x_{2}-x_{1}-x_{3}\right)+x_{1} x_{3}} I_{0,0,1,1,0,0,1,1,2}^{D}\right) \\
& f_{13}^{D}=\epsilon^{3} \sqrt{4-x_{2}} \sqrt{-x_{2}}\left(x_{2}-x_{3}\right) I_{0,1,0,1,0,0,1,1,2}^{D} \\
& f_{14}^{D}=\epsilon^{3} \sqrt{4-x_{2}} \sqrt{-x_{2}} x_{3} I_{0,1,1,0,0,0,1,1,2}^{D} \\
& f_{15}^{D}=\epsilon^{3} \sqrt{4-x_{2}} \sqrt{-x_{2}} x_{1} I_{0,1,1,1,0,0,0,1,2}^{D} \\
& f_{16}^{D}=\epsilon^{3} \sqrt{4-x_{2}} \sqrt{-x_{2}}\left(x_{2}-x_{1}\right) I_{0,1,1,1,0,0,1,0,2}^{D} \\
& f_{17}^{D}=\epsilon^{3} \sqrt{4-x_{2}} \sqrt{-x_{2}} \sqrt{-x_{1}} \sqrt{-x_{3}} \sqrt{4\left(x_{2}-x_{1}-x_{3}\right)+x_{1} x_{3}} I_{0,1,1,1,0,0,1,1,2}^{D}
\end{aligned}
$$




\section{B Pre-canonical master integrals}

In this appendix we draw the 125 master integrals in the pre-canonical form and we link them to the corresponding integral(s) in the canonical basis.

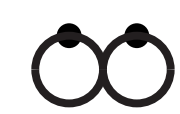

$f_{1}^{A}, f_{1}^{B}, f_{1}^{C}, f_{1}^{D}$

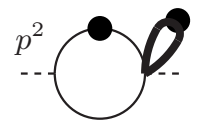

$f_{2}^{B}, f_{11}^{C}$

$f_{2}^{C}$
$f_{8}^{C}$

$q^{2} \bigcirc q^{q^{2}}$

$f_{11}^{B}$
$f_{18}^{C}$

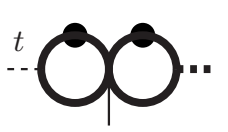

$f_{9}^{D}$

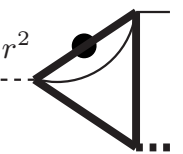

$f_{13}^{A}, f_{15}^{B}, f_{19}^{C}$ $f_{23}^{A}, f_{24}^{B}, f_{15}^{C}$

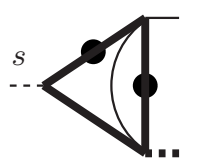

$f_{10}^{A}$

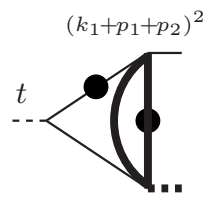

$f_{14}^{C}$

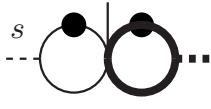

$f_{12}^{B}, f_{24}^{C}$

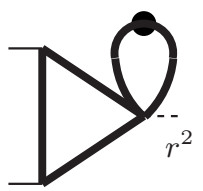

$f_{30}^{A}, f_{7}^{D}$
$f_{6}^{D}$

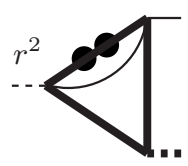

$f_{14}^{A}, f_{16}^{B}, f_{20}^{C}$ $f_{24}^{A}, f_{25}^{B}, f_{16}^{C}$

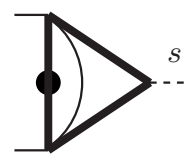

$f_{20}^{A}, f_{19}^{B}$

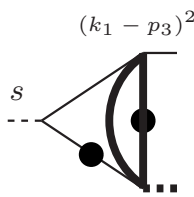

$f_{14}^{B}, f_{23}^{C}$

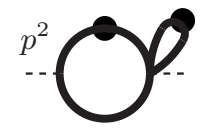

$f_{6}^{A}, f_{7}^{B}, f_{3}^{D}$ $f_{2}^{D}$

$f_{7}^{A}, f_{8}^{B}, f_{7}^{C}, f_{4}^{D}$

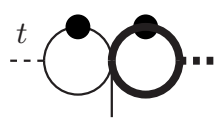

$f_{12}^{C}$

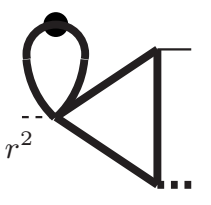

$f_{26}^{A}, f_{22}^{B}, f_{8}^{D}$ $f_{5}^{D}$

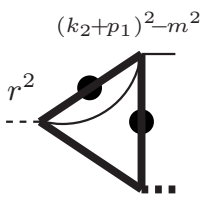

$f_{15}^{A}, f_{17}^{B}, f_{2}^{C}$

$f_{25}^{A}, f_{26}^{B}, f_{17}^{C}$

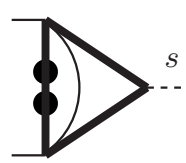

$f_{21}^{A}, f_{20}^{B}$

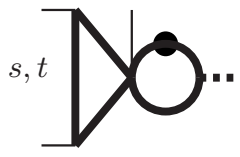

$f_{34}^{A}, f_{15}^{D}$

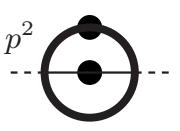

$f_{5}^{A}, f_{3}^{B}, f_{9}^{C}$

$f_{12}^{A}, f_{9}^{B}, f_{3}^{C}$

$f_{3}^{A}, f_{5}^{B}, f_{5}^{C}$

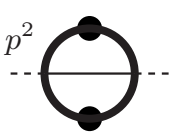

$f_{4}^{A}, f_{4}^{B}, f_{10}^{C}$

$f_{11}^{A}, f_{10}^{B}, f_{4}^{C}$

$f_{2}^{A}, f_{6}^{B}, f_{6}^{C}$

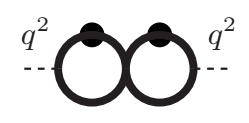

$f_{27}^{A}$
$f_{10}^{D}$

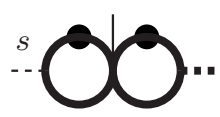

$f_{28}^{A}, f_{11}^{D}$

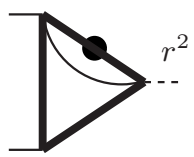

$f_{18}^{A}$

$f_{16}^{A}, f_{23}^{B}$

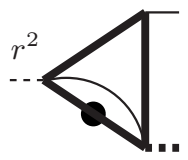

$f_{17}^{A}, f_{18}^{B}$ $f_{19}^{A}$

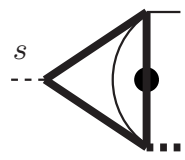

$f_{8}^{A}$

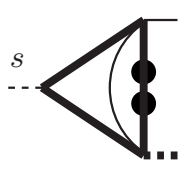

$f_{9}^{A}$

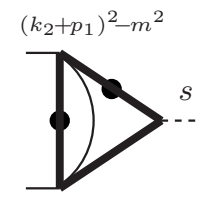

$f_{22}^{A}, f_{21}^{B}$

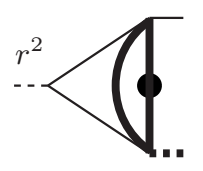

$f_{13}^{B}, f_{22}^{C}$

$f_{13}^{C}$

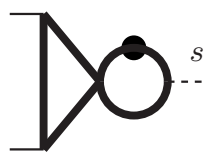

$f_{29}^{A}$

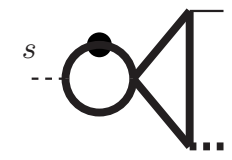

$f_{31}^{A}, f_{16}^{D}$ 
${ }^{s}$

$f_{28}^{B}$

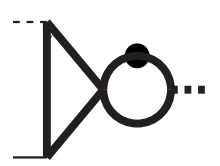

$f_{13}^{D}$

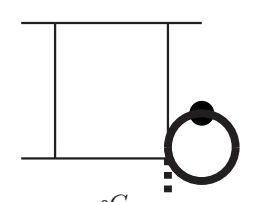

$f_{34}^{C}$

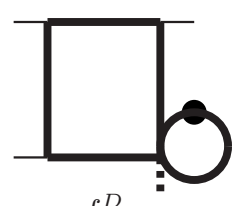

$f_{12}^{D}$

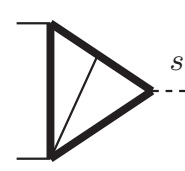

$f_{36}^{A}$

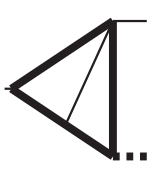

$f_{38}^{A}$

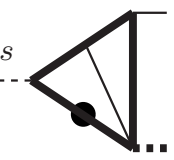

$f_{33}^{A}$
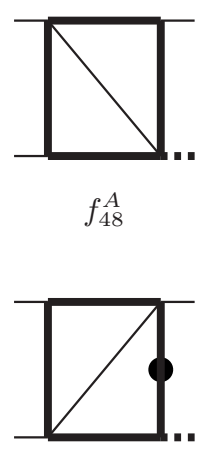

$f_{59}^{A}$

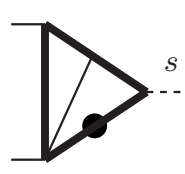

$f_{37}^{A}$

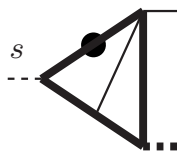

$f_{39}^{A}$

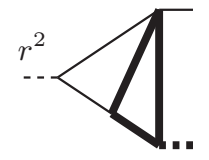

$f_{30}^{B}, f_{37}^{C}$

$f_{25}^{C}$
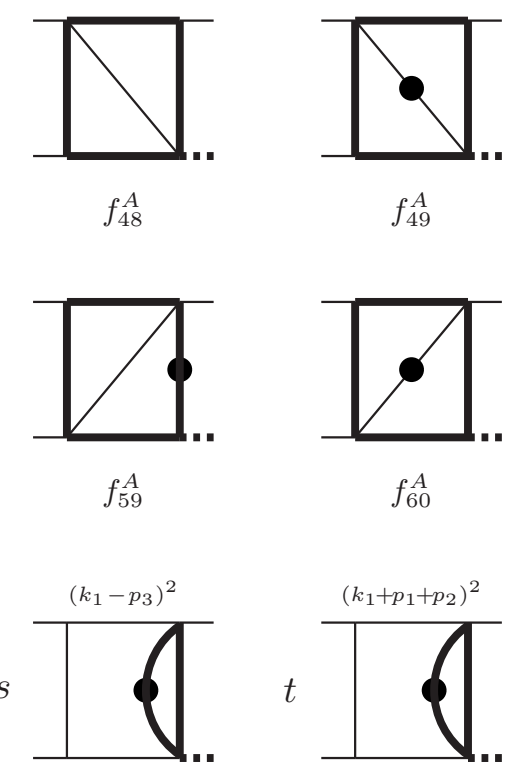

$f_{34}^{B}, f_{36}^{C}$

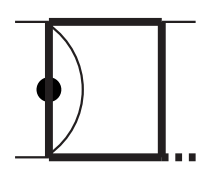

$f_{45}^{A}, f_{38}^{B}$

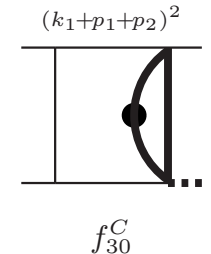

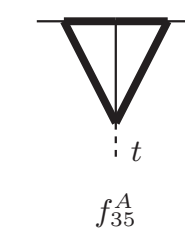
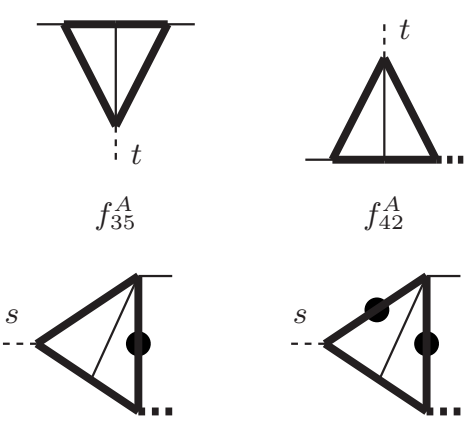

$f_{41}^{A}$

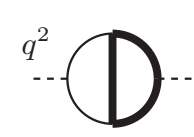

$f_{40}^{A}$

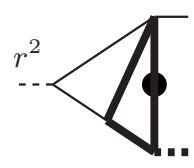

$f_{32}^{B}, f_{39}^{C}$ $f_{27}^{C}$
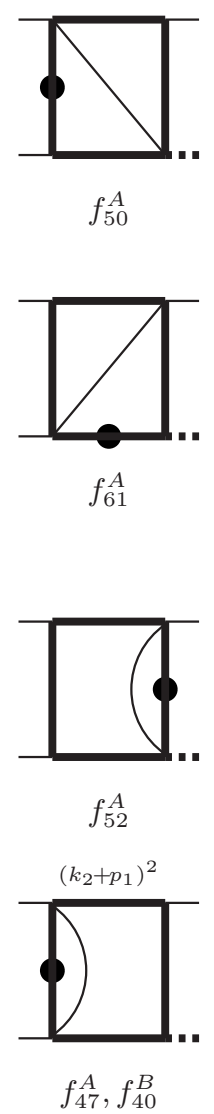

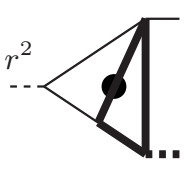

$f_{31}^{B}, f_{38}^{C}$ $f_{26}^{C}$
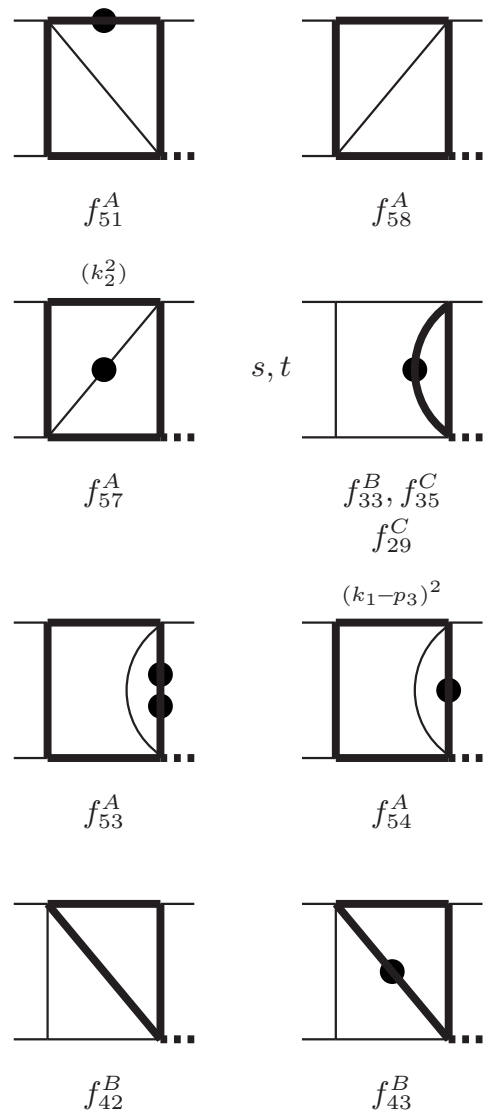


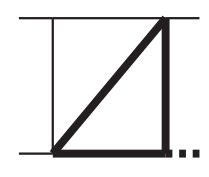

$f_{35}^{B}, f_{31}^{C}$

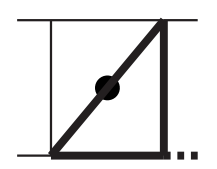

$f_{36}^{B}, f_{32}^{C}$
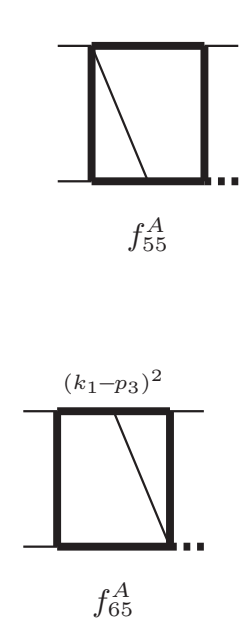

$f_{64}^{A}$

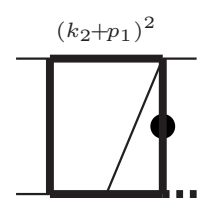

$f_{69}^{A}$

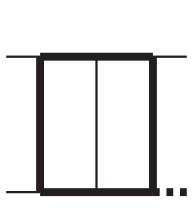

$f_{70}^{A}$
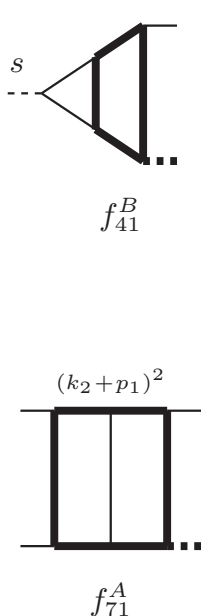

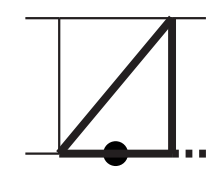

$f_{37}^{B}, f_{33}^{C}$
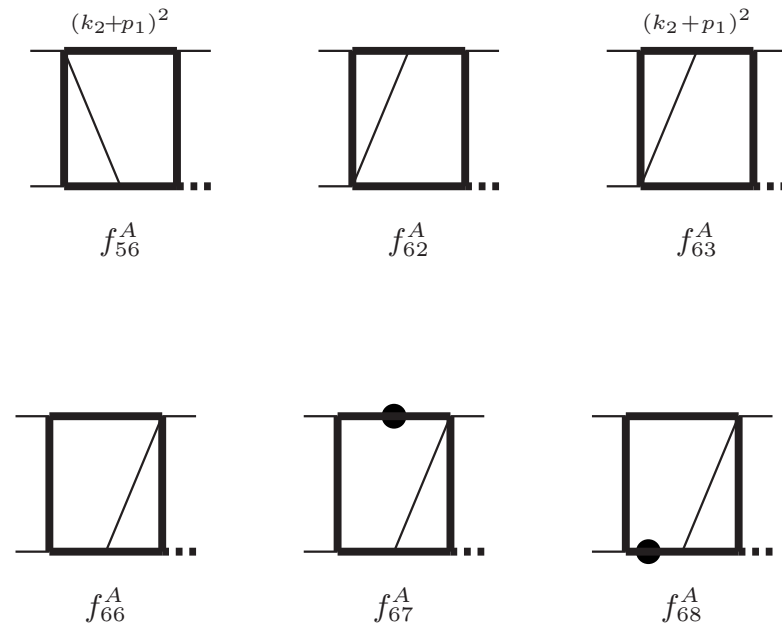

$f_{44}^{A}$
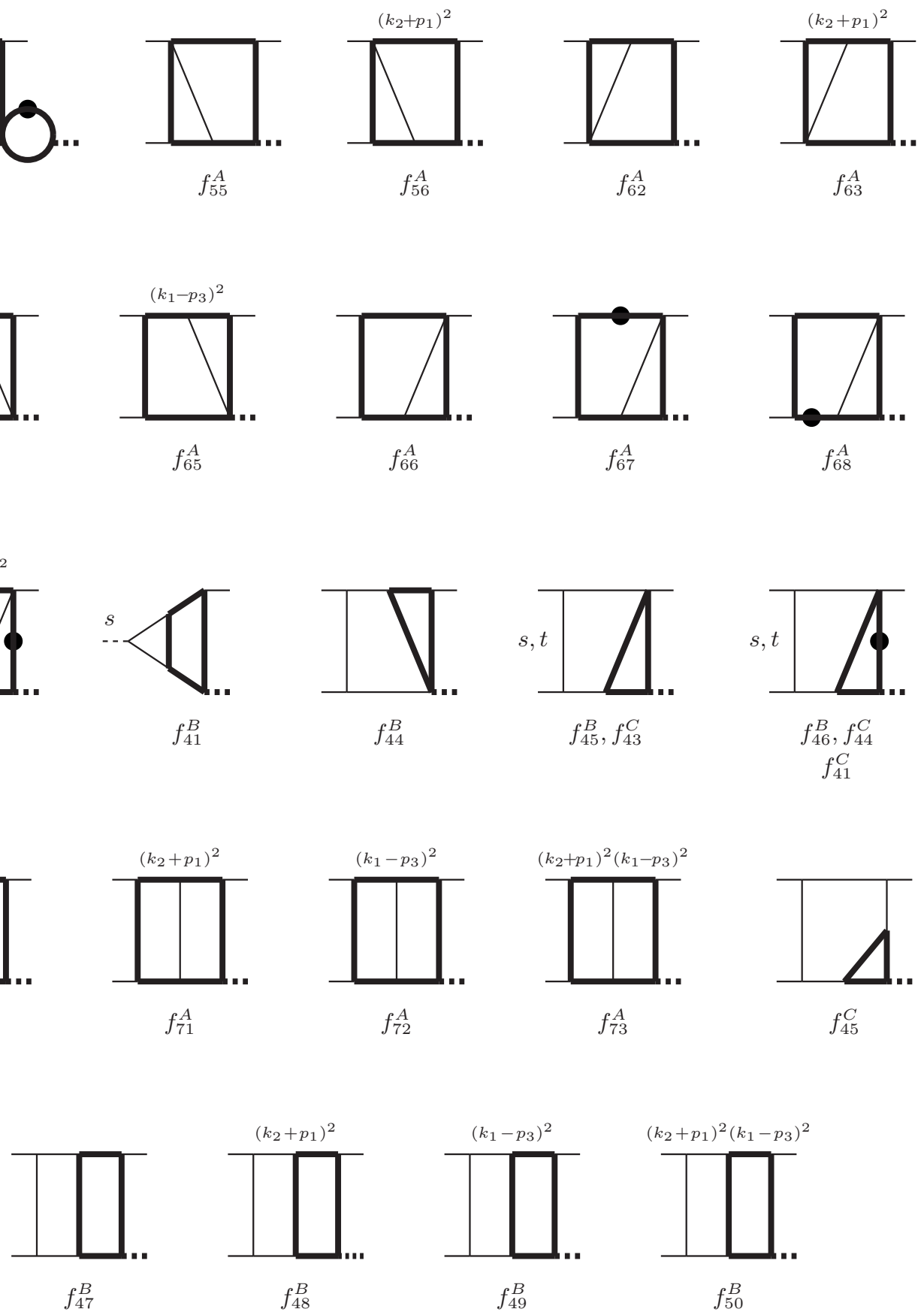


\section{Alphabet}

In this appendix we list the alphabet for the four integral families defined in section 2 . We introduce the following shorthands for the set of 13 square roots,

$$
\begin{array}{rlrl}
R_{1}\left(x_{1}\right) & =\sqrt{-x_{1}}, & R_{1}\left(x_{3}\right)=\sqrt{-x_{3}}, & R_{1}\left(x_{2}\right)=\sqrt{-x_{2}}, \\
R_{2}\left(x_{1}\right) & =\sqrt{4-x_{1}}, & R_{2}\left(x_{3}\right)=\sqrt{4-x_{3}}, & R_{2}\left(x_{2}\right)=\sqrt{4-x_{2}}, \\
R_{3}\left(x_{3}\right)=\sqrt{x_{2}-x_{3}}, & \\
R_{3}\left(x_{1}\right) & =\sqrt{x_{2}-x_{1}}, & & \\
R_{4}\left(x_{3}\right)=\sqrt{x_{2}-x_{3}-4} & \\
R_{4}\left(x_{1}\right) & =\sqrt{x_{2}-x_{1}-4}, & \\
R_{5}(x) & =\sqrt{4 x_{2}+x_{1} x_{3}-4\left(x_{1}+x_{3}\right)}, & & \\
R_{6}(x) & =\sqrt{2 x_{3}\left(-2 x_{2}+x_{1}+2 x_{3}\right)-x_{1} x_{3}^{2}-x_{1}}, \\
R_{7}(x) & =\sqrt{2 x_{1} x_{3}\left(x_{2}-x_{1}\right)+\left(x_{2}-x_{1}\right)^{2}+\left(x_{1}-4\right) x_{1} x_{3}^{2}} .
\end{array}
$$

They appear in the alphabet in the following 8 linearly independent combinations,

$$
\begin{array}{ll}
R_{1}\left(x_{1}\right) R_{2}\left(x_{1}\right), & R_{1}\left(x_{2}\right) R_{2}\left(x_{2}\right), \\
R_{1}\left(x_{3}\right) R_{2}\left(x_{3}\right), & R_{3}\left(x_{1}\right) R_{4}\left(x_{1}\right), \\
R_{3}\left(x_{3}\right) R_{4}\left(x_{3}\right), & R_{1}\left(x_{1}\right) R_{1}\left(x_{3}\right) R_{5}(x), \\
R_{1}\left(x_{1}\right) R_{6}(x), & R_{7}(x) .
\end{array}
$$

Referring to the matrix $\tilde{A}$ defined in (3.4) the alphabets of the four families can be written in terms of the following linearly independent 49 letters,

$$
\begin{aligned}
& \log \left(x_{3}\right), \log \left(x_{1}\right), \log \left(x_{2}\right), \\
& \log \left(x_{1}-4\right), \log \left(x_{3}-4\right), \log \left(x_{2}-4\right), \\
& \log \left(x_{1}+x_{3}\right), \log \left(x_{3}-x_{2}\right), \log \left(x_{1}-x_{2}\right), \\
& \log \left(-x_{2}+x_{1}+x_{3}\right), \log \left(-x_{2}+x_{3}+4\right), \log \left(-x_{2}+x_{1}+4\right), \\
& \log \left(4 x_{2}-4 x_{1}+x_{1} x_{3}-4 x_{3}\right), \log \left(x_{1}^{2}-x_{2} x_{3} x_{1}+2 x_{3} x_{1}+x_{3}^{2}\right), \\
& \log \left(x_{3}^{2}-x_{2} x_{3}+x_{2}\right), \log \left(x_{1}^{2}-x_{2} x_{1}+x_{2}\right), \log \left(x_{2}^{2}-x_{1} x_{2}+x_{1}\right), \\
& \log \left(x_{2}-x_{1}+x_{1} x_{3}+R_{7}(x)\right), \\
& \log \left(x_{2}^{2}-x_{1} x_{2}+x_{1} x_{3} x_{2}-2 x_{1} x_{3}^{2}+R_{7}(x) x_{2}\right), \\
& \log \left(-x_{3} x_{1}^{2}+x_{1}^{2}-x_{2} x_{1}+3 x_{3} x_{1}-R_{7}(x) x_{1}+x_{1}-x_{2}+R_{7}(x)\right), \\
& \log \left(x_{3} x_{1}-x_{1}-2 x_{2} x_{3}+R_{1}\left(x_{1}\right) R_{6}(x)\right), \\
& \log \left(x_{2} x_{1} x_{1}-2 x_{1}-2 x_{3}+R_{1}\left(x_{2}\right) R_{2}\left(x_{2}\right)\right), \\
& \log \left(x_{3}-R_{1}\left(x_{3}\right) R_{2}\left(x_{3}\right)\right), \log \left(x_{1}-R_{1}\left(x_{1}\right) R_{2}\left(x_{1}\right)\right), \\
& \log \left(-x_{3} x_{1}^{2}+x_{1}^{2}-2 x_{2} x_{1}+4 x_{3} x_{1}+R_{2}\left(x_{1}\right) R_{6}(x) x_{1}\right), \\
& \log \left(x_{2}-R_{1}\left(x_{2}\right) R_{2}\left(x_{2}\right)\right), \log \left(x_{2}-x_{3}+R_{3}\left(x_{3}\right) R_{4}\left(x_{3}\right)\right), \\
& \log \left(x_{2}-x_{1}+R_{3}\left(x_{1}\right) R_{4}\left(x_{1}\right)\right), \log \left(x_{2}-2 x_{3}+R_{1}\left(x_{2}\right) R_{2}\left(x_{2}\right)\right), \\
& \log \left(x_{2}-2 x_{1}+R_{1}\left(x_{2}\right) R_{2}\left(x_{2}\right)\right), \log \left(x_{3} x_{1}-x_{1}+R_{1}\left(x_{1}\right) R_{6}(x)\right),
\end{aligned}
$$




$$
\begin{aligned}
& \log \left(-x_{3} x_{1}-x_{1}+R_{1}\left(x_{1}\right) R_{6}(x)\right), \log \left(-x_{2} x_{1}+2 x_{1}+x_{2} R_{1}\left(x_{1}\right) R_{2}\left(x_{1}\right)\right), \\
& \log \left(x_{1} x_{3}+R_{1}\left(x_{1}\right) R_{1}\left(x_{3}\right) R_{5}(x)\right) \\
& \log \left(x_{3} x_{1}-2 x_{1}-2 x_{3}+R_{1}\left(x_{1}\right) R_{1}\left(x_{3}\right) R_{5}(x)\right) \\
& \log \left(x_{3} x_{1}^{2}-x_{1}^{2}+x_{2} x_{1}-4 x_{3} x_{1}+R_{1}\left(x_{1}\right) R_{2}\left(x_{1}\right) R_{7}(x)\right) \\
& \log \left(-x_{2}^{2}+x_{1} x_{2}-x_{1} x_{3} x_{2}+2 x_{3} x_{2}+2 x_{1} x_{3}+R_{1}\left(x_{2}\right) R_{2}\left(x_{2}\right) R_{7}(x)\right) \\
& \log \left(-x_{3}^{2} x_{1}^{2}+3 x_{3} x_{1}^{2}+4 x_{3}^{2} x_{1}-4 x_{2} x_{3} x_{1}+R_{1}\left(x_{3}\right) R_{5}(x) R_{6}(x) x_{1}\right) \\
& \log \left(x_{3} R_{1}\left(x_{2}\right) R_{2}\left(x_{2}\right)+x_{2} R_{1}\left(x_{3}\right) R_{2}\left(x_{3}\right)\right) \\
& \log \left(x_{1} R_{1}\left(x_{2}\right) R_{2}\left(x_{2}\right)+x_{2} R_{1}\left(x_{1}\right) R_{2}\left(x_{1}\right)\right) \\
& \log \left(x_{1} R_{1}\left(x_{3}\right) R_{2}\left(x_{3}\right)-R_{1}\left(x_{1}\right) R_{1}\left(x_{3}\right) R_{5}(x)\right) \\
& \log \left(x_{3} R_{1}\left(x_{1}\right) R_{2}\left(x_{1}\right)-R_{1}\left(x_{1}\right) R_{1}\left(x_{3}\right) R_{5}(x)\right) \\
& \log \left(-x_{2} R_{1}\left(x_{1}\right) R_{2}\left(x_{1}\right)+x_{3} R_{1}\left(x_{1}\right) R_{2}\left(x_{1}\right)+x_{1} R_{3}\left(x_{3}\right) R_{4}\left(x_{3}\right)\right) \\
& \log \left(-x_{2} R_{1}\left(x_{2}\right) R_{2}\left(x_{2}\right)+x_{3} R_{1}\left(x_{2}\right) R_{2}\left(x_{2}\right)+x_{2} R_{3}\left(x_{3}\right) R_{4}\left(x_{3}\right)\right) \\
& \log \left(-x_{2} R_{1}\left(x_{3}\right) R_{2}\left(x_{3}\right)+x_{1} R_{1}\left(x_{3}\right) R_{2}\left(x_{3}\right)+x_{3} R_{3}\left(x_{1}\right) R_{4}\left(x_{1}\right)\right) \\
& \log \left(-x_{2} R_{1}\left(x_{2}\right) R_{2}\left(x_{2}\right)+x_{1} R_{1}\left(x_{2}\right) R_{2}\left(x_{2}\right)+x_{2} R_{3}\left(x_{1}\right) R_{4}\left(x_{1}\right)\right) \\
& \log \left(-x_{3}^{2} x_{1}^{2}+3 x_{3} x_{1}^{2}+4 x_{3}^{2} x_{1}-3 x_{2} x_{3} x_{1}+R_{1}\left(x_{1}\right) R_{1}\left(x_{3}\right) R_{5}(x) R_{7}(x)\right) \\
& \log \left(x_{2} R_{1}\left(x_{1}\right) R_{1}\left(x_{3}\right) R_{5}(x)-x_{1} x_{3} R_{1}\left(x_{2}\right) R_{2}\left(x_{2}\right)\right) \\
& \log \left(-x_{2} x_{3}+x_{1} x_{3}+R_{1}\left(x_{2}\right) R_{2}\left(x_{2}\right) x_{3}-R_{1}\left(x_{1}\right) R_{1}\left(x_{3}\right) R_{5}(x)\right) .
\end{aligned}
$$

\section{Weight-two functions}

In section 3.2 we described how to express the non-elliptic master integrals in terms of a minimal set of logarithms and dilogarithms up to weight two. On the other hand the weightthree functions are one-fold integrals over linear combinations of weight-two functions with algebraic coefficients. Weight-four functions are expressed in two ways. The first consists of logarithms times one-fold integrals over linear combinations of weight-two functions, therefore a function of weight one times one of weight three. The other consists of a one-fold integral of weight-three functions, that are expressed as a product of weight-two functions times logarithms, with algebraic coefficients.

In this appendix we list the basis choice we made for the set of linearly independent dilogarithms required to express the master integrals of each family at weight two. They are chosen to be single-valued in the Euclidean region $x_{3}<x_{2}<x_{1}<0$.

\section{Family A.}

$$
\begin{aligned}
& \operatorname{Li}_{2}\left(\frac{x_{1}}{x_{1}-4}\right), \\
& \operatorname{Li}_{2}\left(\frac{x_{2}}{x_{2}-4}\right), \\
& \operatorname{Li}_{2}\left(\frac{x_{3}}{x_{3}-4}\right), \\
& \operatorname{Li}_{2}\left(\frac{R_{1}\left(x_{3}\right)-R_{2}\left(x_{3}\right)}{R_{1}\left(x_{3}\right)}\right),
\end{aligned}
$$




$$
\begin{aligned}
& \operatorname{Li}_{2}\left(\frac{R_{1}\left(x_{2}\right)-R_{2}\left(x_{2}\right)}{R_{1}\left(x_{2}\right)}\right), \\
& \mathrm{Li}_{2}\left(\frac{R_{1}\left(x_{1}\right)-R_{2}\left(x_{1}\right)}{R_{1}\left(x_{1}\right)}\right), \\
& \operatorname{Li}_{2}\left(\frac{\left(R_{1}\left(x_{3}\right)-R_{2}\left(x_{3}\right)\right)^{2}}{\left(R_{1}\left(x_{3}\right)+R_{2}\left(x_{3}\right)\right)^{2}}\right), \\
& \operatorname{Li}_{2}\left(\frac{\left(R_{1}\left(x_{2}\right)-R_{2}\left(x_{2}\right)\right)^{2}}{\left(R_{1}\left(x_{2}\right)+R_{2}\left(x_{2}\right)\right)^{2}}\right), \\
& \operatorname{Li}_{2}\left(\frac{\left(R_{1}\left(x_{1}\right)-R_{2}\left(x_{1}\right)\right)^{2}}{\left(R_{1}\left(x_{1}\right)+R_{2}\left(x_{1}\right)\right)^{2}}\right), \\
& \operatorname{Li}_{2}\left(\frac{R_{1}\left(x_{1}\right) R_{1}\left(x_{3}\right)-R_{5}(x)}{R_{1}\left(x_{3}\right)\left(R_{1}\left(x_{1}\right)-R_{2}\left(x_{1}\right)\right)}\right), \\
& \operatorname{Li}_{2}\left(\frac{R_{1}\left(x_{3}\right)\left(R_{1}\left(x_{2}\right)-R_{2}\left(x_{2}\right)\right)}{R_{1}\left(x_{2}\right)\left(R_{1}\left(x_{3}\right)+R_{2}\left(x_{3}\right)\right)}\right), \\
& \operatorname{Li}_{2}\left(\frac{R_{1}\left(x_{3}\right)\left(R_{1}\left(x_{2}\right)+R_{2}\left(x_{2}\right)\right)}{R_{1}\left(x_{2}\right)\left(R_{1}\left(x_{3}\right)-R_{2}\left(x_{3}\right)\right)}\right), \\
& \operatorname{Li}_{2}\left(\frac{R_{1}\left(x_{1}\right)\left(R_{1}\left(x_{2}\right)+R_{2}\left(x_{2}\right)\right)}{R_{1}\left(x_{2}\right)\left(R_{1}\left(x_{1}\right)-R_{2}\left(x_{1}\right)\right)}\right) \text {, } \\
& \operatorname{Li}_{2}\left(\frac{R_{1}\left(x_{1}\right)\left(R_{1}\left(x_{2}\right)-R_{2}\left(x_{2}\right)\right)}{R_{1}\left(x_{2}\right)\left(R_{1}\left(x_{1}\right)+R_{2}\left(x_{1}\right)\right)}\right) \text {, } \\
& \operatorname{Li}_{2}\left(\frac{R_{1}\left(x_{1}\right)\left(R_{1}\left(x_{3}\right)+R_{2}\left(x_{3}\right)\right)}{R_{1}\left(x_{1}\right) R_{1}\left(x_{3}\right)-R_{5}(x)}\right) \text {, } \\
& \operatorname{Li}_{2}\left(\frac{R_{1}\left(x_{3}\right)\left(R_{1}\left(x_{1}\right)+R_{2}\left(x_{1}\right)\right)}{R_{1}\left(x_{1}\right) R_{1}\left(x_{3}\right)-R_{5}(x)}\right), \\
& \operatorname{Li}_{2}\left(-\frac{R_{1}\left(x_{1}\right)\left(R_{1}\left(x_{3}\right)-R_{2}\left(x_{3}\right)\right)}{R_{1}\left(x_{1}\right) R_{1}\left(x_{3}\right)-R_{5}(x)}\right), \\
& \mathrm{Li}_{2}\left(\frac{R_{1}\left(x_{1}\right)^{2}\left(R_{1}\left(x_{3}\right)-R_{2}\left(x_{3}\right)\right)^{2}}{\left(R_{1}\left(x_{1}\right) R_{1}\left(x_{3}\right)-R_{5}(x)\right)^{2}}\right), \\
& \operatorname{Li}_{2}\left(\frac{R_{1}\left(x_{1}\right)\left(R_{1}\left(x_{2}\right)-R_{2}\left(x_{2}\right)\right)}{R_{1}\left(x_{2}\right) R_{2}\left(x_{1}\right)-R_{1}\left(x_{1}\right) R_{2}\left(x_{2}\right)}\right), \\
& \operatorname{Li}_{2}\left(\frac{R_{1}\left(x_{2}\right) R_{2}\left(x_{3}\right)-R_{1}\left(x_{3}\right) R_{2}\left(x_{2}\right)}{R_{1}\left(x_{3}\right)\left(R_{1}\left(x_{2}\right)-R_{2}\left(x_{2}\right)\right)}\right), \\
& \mathrm{Li}_{2}\left(-\frac{R_{1}\left(x_{2}\right) R_{2}\left(x_{3}\right)-R_{1}\left(x_{3}\right) R_{2}\left(x_{2}\right)}{R_{1}\left(x_{3}\right)\left(R_{1}\left(x_{2}\right)+R_{2}\left(x_{2}\right)\right)}\right), \\
& \operatorname{Li}_{2}\left(-\frac{R_{1}\left(x_{1}\right)\left(R_{1}\left(x_{2}\right)+R_{2}\left(x_{2}\right)\right)}{R_{1}\left(x_{2}\right) R_{2}\left(x_{1}\right)-R_{1}\left(x_{1}\right) R_{2}\left(x_{2}\right)}\right), \\
& \operatorname{Li}_{2}\left(\frac{R_{1}\left(x_{1}\right) R_{1}\left(x_{3}\right)\left(R_{1}\left(x_{2}\right)+R_{2}\left(x_{2}\right)\right)}{R_{1}\left(x_{2}\right)\left(R_{1}\left(x_{1}\right) R_{1}\left(x_{3}\right)-R_{5}(x)\right)}\right), \\
& \operatorname{Li}_{2}\left(-\frac{R_{1}\left(x_{1}\right) R_{1}\left(x_{3}\right)\left(R_{1}\left(x_{2}\right)-R_{2}\left(x_{2}\right)\right)}{R_{1}\left(x_{2}\right)\left(R_{1}\left(x_{1}\right) R_{1}\left(x_{3}\right)-R_{5}(x)\right)}\right), \\
& \operatorname{Li}_{2}\left(\frac{R_{1}\left(x_{1}\right)^{2} R_{1}\left(x_{3}\right)^{2}\left(R_{1}\left(x_{2}\right)-R_{2}\left(x_{2}\right)\right)^{2}}{R_{1}\left(x_{2}\right)^{2}\left(R_{1}\left(x_{1}\right) R_{1}\left(x_{3}\right)-R_{5}(x)\right)^{2}}\right) .
\end{aligned}
$$




\section{Family B.}

$\operatorname{Li}_{2}\left(\frac{x_{1}}{x_{1}-4}\right)$,

$\operatorname{Li}_{2}\left(\frac{x_{2}}{x_{2}-4}\right)$,

$\operatorname{Li}_{2}\left(\frac{x_{3}}{x_{3}-4}\right)$,

$\operatorname{Li}_{2}\left(\frac{R_{1}\left(x_{3}\right)-R_{2}\left(x_{3}\right)}{R_{1}\left(x_{3}\right)}\right)$,

$\operatorname{Li}_{2}\left(\frac{R_{1}\left(x_{2}\right)-R_{2}\left(x_{2}\right)}{R_{1}\left(x_{2}\right)}\right)$,

$\operatorname{Li}_{2}\left(\frac{R_{1}\left(x_{1}\right)-R_{2}\left(x_{1}\right)}{R_{1}\left(x_{1}\right)}\right)$,

$\operatorname{Li}_{2}\left(\frac{\left(R_{1}\left(x_{3}\right)-R_{2}\left(x_{3}\right)\right)^{2}}{\left(R_{1}\left(x_{3}\right)+R_{2}\left(x_{3}\right)\right)^{2}}\right)$,

$\operatorname{Li}_{2}\left(\frac{\left(R_{1}\left(x_{2}\right)-R_{2}\left(x_{2}\right)\right)^{2}}{\left(R_{1}\left(x_{2}\right)+R_{2}\left(x_{2}\right)\right)^{2}}\right)$,

$\operatorname{Li}_{2}\left(\frac{\left(R_{1}\left(x_{1}\right)-R_{2}\left(x_{1}\right)\right)^{2}}{\left(R_{1}\left(x_{1}\right)+R_{2}\left(x_{1}\right)\right)^{2}}\right)$,

$\operatorname{Li}_{2}\left(\frac{R_{1}\left(x_{3}\right)\left(R_{1}\left(x_{2}\right)-R_{2}\left(x_{2}\right)\right)}{R_{1}\left(x_{2}\right)\left(R_{1}\left(x_{3}\right)+R_{2}\left(x_{3}\right)\right)}\right)$,

$\operatorname{Li}_{2}\left(\frac{R_{1}\left(x_{3}\right)\left(R_{1}\left(x_{2}\right)+R_{2}\left(x_{2}\right)\right)}{R_{1}\left(x_{2}\right)\left(R_{1}\left(x_{3}\right)-R_{2}\left(x_{3}\right)\right)}\right)$,

$\operatorname{Li}_{2}\left(\frac{R_{1}\left(x_{1}\right)\left(R_{1}\left(x_{2}\right)+R_{2}\left(x_{2}\right)\right)}{R_{1}\left(x_{2}\right)\left(R_{1}\left(x_{1}\right)-R_{2}\left(x_{1}\right)\right)}\right)$,

$\operatorname{Li}_{2}\left(\frac{R_{1}\left(x_{1}\right)\left(R_{1}\left(x_{2}\right)-R_{2}\left(x_{2}\right)\right)}{R_{1}\left(x_{2}\right)\left(R_{1}\left(x_{1}\right)+R_{2}\left(x_{1}\right)\right)}\right)$,

$\mathrm{Li}_{2}\left(\frac{x_{2}+R_{1}\left(x_{2}\right) R_{2}\left(x_{2}\right)-2}{-x_{1}+x_{2}+R_{3}(x) R_{4}(x)-2}\right)$,

$\operatorname{Li}_{2}\left(\frac{R_{1}\left(x_{1}\right)\left(R_{1}\left(x_{2}\right)-R_{2}\left(x_{2}\right)\right)}{R_{1}\left(x_{2}\right) R_{2}\left(x_{1}\right)-R_{1}\left(x_{1}\right) R_{2}\left(x_{2}\right)}\right)$,

$\operatorname{Li}_{2}\left(\frac{R_{1}\left(x_{2}\right) R_{2}\left(x_{3}\right)-R_{1}\left(x_{3}\right) R_{2}\left(x_{2}\right)}{R_{1}\left(x_{3}\right)\left(R_{1}\left(x_{2}\right)-R_{2}\left(x_{2}\right)\right)}\right)$,

$\operatorname{Li}_{2}\left(-\frac{R_{1}\left(x_{1}\right)\left(R_{1}\left(x_{2}\right)+R_{2}\left(x_{2}\right)\right)}{R_{1}\left(x_{2}\right) R_{2}\left(x_{1}\right)-R_{1}\left(x_{1}\right) R_{2}\left(x_{2}\right)}\right)$,

$\mathrm{Li}_{2}\left(-\frac{R_{1}\left(x_{2}\right) R_{2}\left(x_{3}\right)-R_{1}\left(x_{3}\right) R_{2}\left(x_{2}\right)}{R_{1}\left(x_{3}\right)\left(R_{1}\left(x_{2}\right)+R_{2}\left(x_{2}\right)\right)}\right)$,

$\operatorname{Li}_{2}\left(\frac{R_{1}\left(x_{1}\right) R_{1}\left(x_{3}\right)\left(x_{3}-R_{1}\left(x_{3}\right) R_{2}\left(x_{3}\right)\right)}{x_{3}\left(R_{1}\left(x_{1}\right) R_{1}\left(x_{3}\right)-R_{5}(x)\right)}\right)$,

$\operatorname{Li}_{2}\left(\frac{R_{1}\left(x_{1}\right) R_{1}\left(x_{3}\right)\left(x_{2}-R_{1}\left(x_{2}\right) R_{2}\left(x_{2}\right)\right)}{x_{2}\left(R_{1}\left(x_{1}\right) R_{1}\left(x_{3}\right)-R_{5}(x)\right)}\right)$, 


$$
\begin{aligned}
& \operatorname{Li}_{2}\left(\frac{R_{1}\left(x_{1}\right) R_{1}\left(x_{3}\right)\left(x_{1}-R_{1}\left(x_{1}\right) R_{2}\left(x_{1}\right)\right)}{x_{1}\left(R_{1}\left(x_{1}\right) R_{1}\left(x_{3}\right)-R_{5}(x)\right)}\right), \\
& \operatorname{Li}_{2}\left(\frac{R_{1}\left(x_{1}\right) R_{1}\left(x_{3}\right)\left(R_{1}\left(x_{2}\right)-R_{2}\left(x_{2}\right)\right)}{R_{1}\left(x_{2}\right) R_{5}(x)-R_{1}\left(x_{1}\right) R_{1}\left(x_{3}\right) R_{2}\left(x_{2}\right)}\right), \\
& \operatorname{Li}_{2}\left(-\frac{R_{1}\left(x_{1}\right) R_{1}\left(x_{3}\right)\left(x_{1}+R_{1}\left(x_{1}\right) R_{2}\left(x_{1}\right)\right)}{x_{1}\left(R_{1}\left(x_{1}\right) R_{1}\left(x_{3}\right)-R_{5}(x)\right)}\right), \\
& \operatorname{Li}_{2}\left(-\frac{R_{1}\left(x_{1}\right) R_{1}\left(x_{3}\right)\left(x_{3}+R_{1}\left(x_{3}\right) R_{2}\left(x_{3}\right)\right)}{x_{3}\left(R_{1}\left(x_{1}\right) R_{1}\left(x_{3}\right)-R_{5}(x)\right)}\right), \\
& \operatorname{Li}_{2}\left(-\frac{x_{1}\left(R_{1}\left(x_{1}\right) R_{1}\left(x_{3}\right)-R_{5}(x)\right)^{2}}{R_{1}\left(x_{1}\right)^{2} R_{1}\left(x_{3}\right)^{2}\left(R_{1}\left(x_{1}\right)-R_{2}\left(x_{1}\right)\right)^{2}}\right), \\
& \operatorname{Li}_{2}\left(\frac{2 R_{1}\left(x_{1}\right)^{2} R_{1}\left(x_{3}\right)^{2}\left(x_{3}+R_{1}\left(x_{3}\right) R_{2}\left(x_{3}\right)-2\right)}{x_{3}\left(R_{1}\left(x_{1}\right) R_{1}\left(x_{3}\right)-R_{5}(x)\right)^{2}}\right), \\
& \operatorname{Li}_{2}\left(\frac{x_{1} x_{2}\left(R_{1}\left(x_{2}\right)+R_{2}\left(x_{2}\right)\right)}{-R_{3}(x) R_{4}(x) R_{1}\left(x_{2}\right)^{3}-x_{2}^{2} R_{2}\left(x_{2}\right)+x_{1} x_{2} R_{2}\left(x_{2}\right)}\right), \\
& \operatorname{Li}_{2}\left(-\frac{\left(x_{1}-x_{2}\right) x_{2}\left(R_{1}\left(x_{2}\right)-R_{2}\left(x_{2}\right)\right)}{-R_{3}(x) R_{4}(x) R_{1}\left(x_{2}\right)^{3}-x_{2}^{2} R_{2}\left(x_{2}\right)+x_{1} x_{2} R_{2}\left(x_{2}\right)}\right) .
\end{aligned}
$$

\section{Family C.}

$$
\begin{aligned}
& \mathrm{Li}_{2}\left(\frac{1-x_{2}}{x_{1}}\right), \\
& \mathrm{Li}_{2}\left(\frac{1-x_{2}}{x_{3}}\right), \\
& \mathrm{Li}_{2}\left(\frac{x_{1}}{x_{1}-x_{2}+x_{3}}\right), \\
& \mathrm{Li}_{2}\left(\frac{x_{1} x_{3}}{x_{2}\left(x_{1}-x_{2}+x_{3}\right)}\right), \\
& \operatorname{Li}_{2}\left(\frac{\left(R_{1}\left(x_{2}\right)+R_{2}\left(x_{2}\right)\right)^{2}}{\left(R_{1}\left(x_{3}\right)+R_{2}\left(x_{3}\right)\right)^{2}}\right), \\
& \operatorname{Li}_{2}\left(\frac{\left(R_{1}\left(x_{1}\right)+R_{2}\left(x_{1}\right)\right)^{2}}{\left(R_{1}\left(x_{2}\right)+R_{2}\left(x_{2}\right)\right)^{2}}\right), \\
& \operatorname{Li}_{2}\left(\frac{-4}{\left(R_{3}\left(x_{1}\right)+R_{4}\left(x_{1}\right)\right)^{2}}\right), \\
& \operatorname{Li}_{2}\left(\frac{16}{\left(R_{1}\left(x_{3}\right)+R_{2}\left(x_{3}\right)\right)^{4}}\right), \\
& \operatorname{Li}_{2}\left(\frac{-4}{\left(R_{1}\left(x_{3}\right)+R_{2}\left(x_{3}\right)\right)^{2}}\right), \\
& \operatorname{Li}_{2}\left(\frac{16}{\left(R_{1}\left(x_{2}\right)+R_{2}\left(x_{2}\right)\right)^{4}}\right), \\
& \operatorname{Li}_{2}\left(\frac{-4}{\left(R_{1}\left(x_{2}\right)+R_{2}\left(x_{2}\right)\right)^{2}}\right), \\
& \operatorname{Li}_{2}\left(\frac{16}{\left(R_{1}\left(x_{1}\right)+R_{2}\left(x_{1}\right)\right)^{4}}\right),
\end{aligned}
$$




$$
\begin{aligned}
& \operatorname{Li}_{2}\left(\frac{-4}{\left(R_{1}\left(x_{1}\right)+R_{2}\left(x_{1}\right)\right)^{2}}\right), \\
& \operatorname{Li}_{2}\left(\frac{-4}{\left(R_{3}\left(x_{3}\right)+R_{4}\left(x_{3}\right)\right)^{2}}\right) \text {, } \\
& \operatorname{Li}_{2}\left(\frac{-4\left(x_{1}-x_{2}+x_{3}\right)}{\left(R_{1}\left(x_{1}\right) R_{1}\left(x_{3}\right)+R_{5}(x)\right)^{2}}\right), \\
& \operatorname{Li}_{2}\left(\frac{R_{1}\left(x_{1}\right)\left(R_{1}\left(x_{3}\right)+R_{2}\left(x_{3}\right)\right)}{R_{1}\left(x_{1}\right) R_{1}\left(x_{3}\right)+R_{5}(x)}\right), \\
& \operatorname{Li}_{2}\left(\frac{R_{1}\left(x_{2}\right)\left(R_{1}\left(x_{2}\right)+R_{2}\left(x_{2}\right)\right)}{R_{1}\left(x_{3}\right)\left(R_{1}\left(x_{3}\right)+R_{2}\left(x_{3}\right)\right)}\right), \\
& \operatorname{Li}_{2}\left(\frac{R_{1}\left(x_{1}\right) R_{1}\left(x_{3}\right)\left(R_{1}\left(x_{2}\right)+R_{2}\left(x_{2}\right)\right)}{R_{1}\left(x_{2}\right)\left(R_{1}\left(x_{1}\right) R_{1}\left(x_{3}\right)+R_{5}(x)\right)}\right), \\
& \operatorname{Li}_{2}\left(\frac{-x_{1}\left(R_{1}\left(x_{2}\right)+R_{2}\left(x_{2}\right)\right)^{2}}{\left(R_{2}\left(x_{2}\right) R_{3}\left(x_{1}\right)+R_{1}\left(x_{2}\right) R_{4}\left(x_{1}\right)\right)^{2}}\right) \text {, } \\
& \operatorname{Li}_{2}\left(\frac{-\left(x_{1}-x_{2}+x_{3}\right)\left(R_{1}\left(x_{1}\right)+R_{2}\left(x_{1}\right)\right)^{2}}{\left.R_{1}\left(x_{1}\right) R_{1}\left(x_{3}\right)+R_{5}(x)\right)^{2}}\right) \text {, } \\
& \operatorname{Li}_{2}\left(\frac{16}{\left(R_{1}\left(x_{1}\right)+R_{2}\left(x_{1}\right)\right)^{2}\left(R_{1}\left(x_{2}\right)+R_{2}\left(x_{2}\right)\right)^{2}}\right), \\
& \mathrm{Li}_{2}\left(\frac{16}{\left(R_{1}\left(x_{2}\right)+R_{2}\left(x_{2}\right)\right)^{2}\left(R_{1}\left(x_{3}\right)+R_{2}\left(x_{3}\right)\right)^{2}}\right) \text {, } \\
& \operatorname{Li}_{2}\left(\frac{-x_{1}\left(R_{1}\left(x_{2}\right)+R_{2}\left(x_{2}\right)\right)}{R_{3}\left(x_{1}\right)\left(R_{2}\left(x_{2}\right) R_{3}\left(x_{1}\right)+R_{1}\left(x_{2}\right) R_{4}\left(x_{1}\right)\right)}\right) \text {, } \\
& \operatorname{Li}_{2}\left(\frac{-4 R_{1}\left(x_{1}\right)}{\left(R_{1}\left(x_{3}\right)+R_{2}\left(x_{3}\right)\right)\left(R_{1}\left(x_{1}\right) R_{1}\left(x_{3}\right)+R_{5}(x)\right)}\right) \text {, } \\
& \operatorname{Li}_{2}\left(\frac{-4 R_{1}\left(x_{2}\right)}{R_{1}\left(x_{3}\right)\left(R_{1}\left(x_{2}\right)+R_{2}\left(x_{2}\right)\right)\left(R_{1}\left(x_{3}\right)+R_{2}\left(x_{3}\right)\right)}\right), \\
& \operatorname{Li}_{2}\left(\frac{-16\left(x_{1}-x_{2}+x_{3}\right)}{\left(R_{1}\left(x_{1}\right)+R_{2}\left(x_{1}\right)\right)^{2}\left(R_{1}\left(x_{1}\right) R_{1}\left(x_{3}\right)+R_{5}(x)\right)^{2}}\right), \\
& \mathrm{Li}_{2}\left(\frac{-4 R_{1}\left(x_{1}\right) R_{1}\left(x_{3}\right)}{R_{1}\left(x_{2}\right)\left(R_{1}\left(x_{2}\right)+R_{2}\left(x_{2}\right)\right)\left(R_{1}\left(x_{1}\right) R_{1}\left(x_{3}\right)+R_{5}(x)\right)}\right), \\
& \operatorname{Li}_{2}\left(\frac{4 R_{3}\left(x_{3}\right)}{\left(R_{1}\left(x_{2}\right)+R_{2}\left(x_{2}\right)\right)\left(R_{2}\left(x_{2}\right) R_{3}\left(x_{3}\right)+R_{1}\left(x_{2}\right) R_{4}\left(x_{3}\right)\right)}\right), \\
& \operatorname{Li}_{2}\left(\frac{-16 x_{1}}{\left(R_{1}\left(x_{2}\right)+R_{2}\left(x_{2}\right)\right)^{2}\left(R_{2}\left(x_{2}\right) R_{3}\left(x_{1}\right)+R_{1}\left(x_{2}\right) R_{4}\left(x_{1}\right)\right)^{2}}\right), \\
& \mathrm{Li}_{2}\left(\frac{-4 x_{1}}{\left(R_{1}\left(x_{2}\right)+R_{2}\left(x_{2}\right)\right) R_{3}\left(x_{1}\right)\left(R_{2}\left(x_{2}\right) R_{3}\left(x_{1}\right)+R_{1}\left(x_{2}\right) R_{4}\left(x_{1}\right)\right)}\right) \text {, } \\
& \mathrm{Li}_{2}\left(\frac{-4 x_{3}}{\left(R_{1}\left(x_{2}\right)+R_{2}\left(x_{2}\right)\right) R_{3}\left(x_{3}\right)\left(R_{2}\left(x_{2}\right) R_{3}\left(x_{3}\right)+R_{1}\left(x_{2}\right) R_{4}\left(x_{3}\right)\right)}\right) \text {. }
\end{aligned}
$$




\section{Family D.}

$$
\begin{aligned}
& \operatorname{Li}_{2}\left(\frac{x_{2}-4}{x_{3}-4}\right), \\
& \mathrm{Li}_{2}\left(\frac{x_{1}-4}{x_{2}-4}\right) \text {, } \\
& \operatorname{Li}_{2}\left(\frac{R_{1}\left(x_{3}\right)}{R_{2}\left(x_{3}\right)}\right) \text {, } \\
& \mathrm{Li}_{2}\left(-\frac{R_{1}\left(x_{3}\right)}{R_{2}\left(x_{3}\right)}\right) \text {, } \\
& \mathrm{Li}_{2}\left(\frac{R_{5}(x)}{R_{1}\left(x_{3}\right) R_{2}\left(x_{1}\right)}\right), \\
& \operatorname{Li}_{2}\left(-\frac{R_{5}(x)}{R_{1}\left(x_{3}\right) R_{2}\left(x_{1}\right)}\right), \\
& \operatorname{Li}_{2}\left(\frac{R_{2}\left(x_{2}\right)}{R_{1}\left(x_{2}\right)+R_{2}\left(x_{2}\right)}\right), \\
& \operatorname{Li}_{2}\left(\frac{R_{2}\left(x_{1}\right)}{R_{1}\left(x_{1}\right)+R_{2}\left(x_{1}\right)}\right) \text {, } \\
& \operatorname{Li}_{2}\left(-\frac{4\left(x_{2}-4\right)}{\left(x_{1}-4\right)\left(x_{3}-4\right)}\right) \text {, } \\
& \operatorname{Li}_{2}\left(\frac{16}{\left(R_{1}\left(x_{1}\right)+R_{2}\left(x_{1}\right)\right)^{4}}\right) \text {, } \\
& \operatorname{Li}_{2}\left(\frac{16}{\left(R_{1}\left(x_{2}\right)+R_{2}\left(x_{2}\right)\right)^{4}}\right) \text {, } \\
& \operatorname{Li}_{2}\left(\frac{16}{\left(R_{1}\left(x_{3}\right)+R_{2}\left(x_{3}\right)\right)^{4}}\right), \\
& \operatorname{Li}_{2}\left(\frac{R_{1}\left(x_{1}\right) R_{1}\left(x_{3}\right) R_{2}\left(x_{2}\right)}{R_{1}\left(x_{2}\right) R_{5}(x)}\right), \\
& \mathrm{Li}_{2}\left(\frac{R_{1}\left(x_{1}\right) R_{1}\left(x_{3}\right)+R_{5}(x)}{R_{1}\left(x_{3}\right) R_{2}\left(x_{1}\right)+R_{5}(x)}\right), \\
& \mathrm{Li}_{2}\left(\frac{R_{1}\left(x_{1}\right) R_{1}\left(x_{3}\right)+R_{5}(x)}{R_{1}\left(x_{1}\right) R_{2}\left(x_{3}\right)+R_{5}(x)}\right), \\
& \mathrm{Li}_{2}\left(\frac{R_{5}(x)}{R_{1}\left(x_{1}\right) R_{1}\left(x_{3}\right)+R_{5}(x)}\right) \text {, } \\
& \mathrm{Li}_{2}\left(\frac{R_{5}(x)}{R_{1}\left(x_{1}\right) R_{2}\left(x_{3}\right)+R_{5}(x)}\right), \\
& \mathrm{Li}_{2}\left(-\frac{R_{1}\left(x_{2}\right) R_{5}(x)}{R_{1}\left(x_{1}\right) R_{1}\left(x_{3}\right) R_{2}\left(x_{2}\right)}\right), \\
& \operatorname{Li}_{2}\left(\frac{4}{R_{2}\left(x_{1}\right)\left(R_{1}\left(x_{1}\right)+R_{2}\left(x_{1}\right)\right)}\right), \\
& \operatorname{Li}_{2}\left(\frac{4}{R_{2}\left(x_{2}\right)\left(R_{1}\left(x_{2}\right)+R_{2}\left(x_{2}\right)\right)}\right) \text {, }
\end{aligned}
$$




$$
\begin{aligned}
& \operatorname{Li}_{2}\left(\frac{R_{1}\left(x_{1}\right) R_{1}\left(x_{3}\right)+R_{5}(x)}{R_{1}\left(x_{3}\right)\left(R_{1}\left(x_{1}\right)+R_{2}\left(x_{1}\right)\right)}\right), \\
& \operatorname{Li}_{2}\left(\frac{R_{2}\left(x_{3}\right)\left(R_{1}\left(x_{1}\right) R_{1}\left(x_{3}\right)+R_{5}(x)\right)}{\left(R_{1}\left(x_{3}\right)+R_{2}\left(x_{3}\right)\right) R_{5}(x)}\right), \\
& \operatorname{Li}_{2}\left(\frac{R_{1}\left(x_{1}\right) R_{1}\left(x_{3}\right)\left(R_{1}\left(x_{2}\right)+R_{2}\left(x_{2}\right)\right)}{R_{1}\left(x_{2}\right)\left(R_{1}\left(x_{1}\right) R_{1}\left(x_{3}\right)+R_{5}(x)\right)}\right), \\
& \operatorname{Li}_{2}\left(\frac{R_{1}\left(x_{2}\right)\left(R_{1}\left(x_{1}\right) R_{1}\left(x_{3}\right)+R_{5}(x)\right)}{R_{1}\left(x_{1}\right) R_{1}\left(x_{3}\right) R_{2}\left(x_{2}\right)+R_{1}\left(x_{2}\right) R_{5}(x)}\right) .
\end{aligned}
$$

\section{E One-fold integral representations}

We consider a system of differential equations for a set of integrals $f(x, \epsilon)$ in canonical form [34] defined by a matrix $\tilde{A}(x)$,

$$
d f^{(i)}(x)=d \tilde{A}(x) f^{(i-1)}(x) .
$$

If some boundary values $f^{(i)}(0)$ and a parametrization of the integration path are provided, the equations can be readily integrated. The integration path goes from the boundary point to $x$. If the boundary point is $x=0$ a convenient parametrization is $x(\alpha)=x \alpha$ with $\alpha \in[0,1]$. The solution reads

$$
f^{(i)}(x)=\int_{0}^{1} d \alpha\left(\partial_{\alpha} \tilde{A}(\alpha)\right) f^{(i-1)}(\alpha)+f^{(i)}(0) .
$$

Performing an integration by parts we can reduce the weight of the functions involved,

$$
\begin{aligned}
f^{(i)}(x)= & \tilde{A}(1)\left(\int_{0}^{1} d \alpha\left(\partial_{\alpha} \tilde{A}(\alpha)\right) f^{(i-2)}(\alpha)+f^{(i-1)}(0)\right)-\tilde{A}(0) f^{(i-1)}(0) \\
& -\int_{0}^{1} d \alpha \tilde{A}(\alpha)\left(\partial_{\alpha} \tilde{A}(\alpha)\right) f^{(i-2)}(\alpha)+f^{(i)}(0) .
\end{aligned}
$$

If the weight-two functions are known analytically, weight-three functions can be computed numerically using eq. (E.2), while the weight-four functions are computed via eq. (E.3). In general the matrix $\tilde{A}(x)$ and the functions $f(x, \epsilon)$ may have singular behavior for $x \rightarrow 0$ $(\alpha \rightarrow 0)$, so that one has to distinguish different cases in order to properly define the previous expression. If the boundary values are $f(0, \epsilon)=0$ (as for most of the integrals discussed in this paper, see section 3.2), eq. (E.3) is well-defined (this is the case also if $\tilde{A}(0)$ is singular, since $f^{(i-1)}(0)$ vanish in the same limit and the second term on the right hand side vanishes).

On the other hand, when $f(0, \epsilon)$ is singular, eq. (E.3) is not defined. Nevertheless in our case all the divergent integrals are factorisable into products of one-loop integrals, which are already known analytically to all orders of $\epsilon[24,79]$. We then need to define the integrals that, via eq. (E.3), depend on those with singular boundary values. Assume that integral $f_{k}(x, \epsilon)$ has a singular boundary condition $f_{k}(0, \epsilon)$, and that it is known analytically to all orders of $\epsilon$. Consider an integral $f_{n}(x, \epsilon)$, with $n \neq k$, with a regular boundary condition 
$f_{n}(0, \epsilon)$. Using eq. (E.2) we can write it as,

$$
f_{n}^{(i)}(x)=\sum_{m \neq k} \int_{0}^{1} d \alpha\left(\partial_{\alpha} \tilde{A}_{n m}(\alpha)\right) f_{m}^{(i-1)}(\alpha)+\int_{0}^{1} d \alpha\left(\partial_{\alpha} \tilde{A}_{n k}(\alpha)\right) f_{k}^{(i-1)}(\alpha)+f_{n}^{(i)}(0),
$$

Since by assumption $f_{k}^{(i-1)}(x)$ is known analytically, we can directly evaluate the second integral on the right hand side. Also, the fact that $f_{n}(0, \epsilon)$ is regular ensures that the second integral is well-defined even if $f_{k}^{(i-1)}(0)$ is singular. Finally we can perform an integration by parts and reduce the other integrals to the form of (E.3).

Another exception is represented by integrals with regular but non-zero boundary conditions, since the term $\tilde{A}(0) f^{(i-1)}(0)$ of eq. (E.3) would be ill-defined if $\tilde{A}(0)$ was singular. Again, in our case integrals with non-zero boundary conditions are factorized ones, so that they are already known analytically to all orders, and we can proceed as explained above for the case of singular boundary conditions.

\section{F Maximal cut of the elliptic sectors}

We show that the maximal cut $[35,89]$ of $I_{1,1,0,1,1,1,1,0,0}^{A}$ provides useful information about the class of functions needed to represent the result. We cut the six visible propagators. We parametrize the two loop momenta using the spinor-helicity formalism [90] (see [91, 92] for a different formalism),

$$
\begin{aligned}
& k_{1}^{\mu}=z_{1} p_{1}^{\mu}+z_{2} p_{2}^{\mu}+z_{3} \frac{\left\langle 1^{-}\left|\gamma^{\mu}\right| 2^{-}\right\rangle}{2\langle 13\rangle[32]}+z_{4} \frac{\left\langle 2^{-}\left|\gamma^{\mu}\right| 1^{-}\right\rangle}{2\langle 23\rangle[31]}, \\
& k_{2}^{\mu}=z_{5} p_{1}^{\mu}+z_{6} p_{2}^{\mu}+z_{7} \frac{\left\langle 1^{-}\left|\gamma^{\mu}\right| 2^{-}\right\rangle}{2\langle 13\rangle[32]}+z_{8} \frac{\left\langle 2^{-}\left|\gamma^{\mu}\right| 1^{-}\right\rangle}{2\langle 23\rangle[31]} .
\end{aligned}
$$

We get the following two-fold integral result for the maximal cut,

$$
\bar{I}=\frac{s_{13} s_{23}}{s_{12}^{2}} \int d z_{6} d z_{8} \frac{1}{\sqrt{F_{1} F_{2}}},
$$

where the two factors under the square root are,

$$
\begin{aligned}
& F_{1}=m^{2} s_{13} s_{23}-s_{12} z_{8}\left(\left(s_{12}+s_{23}\right) z_{6}-s_{13}+z_{8}\right), \\
& F_{2}=m^{2} s_{13} s_{23}\left(2 z_{6}+1\right)^{2}+4 m^{2}\left(s_{12}+s_{13}\right) z_{6} z_{8}-s_{12} z_{8}\left(\left(s_{12}+s_{23}\right) z_{6}-s_{13}+z_{8}\right) .
\end{aligned}
$$

The integrand is the square root of a quartic polynomial in $z_{8}$, with four different roots. This means that the integrand has two genuine branch cuts that cannot be removed by any change of variables, yielding an elliptic integral upon integration [59, 93].

For completeness let us also show that localizing the two loops individually gives a consistent result. First we may localize the integration momentum $k_{1}$ by cutting propagators 1,2,5,6 (using the numbering of (A.1)). This yields the result,

$$
\bar{I}_{\text {box-cut }}=s_{12}^{2} \int \frac{d^{4} k_{2}}{\left(i \pi^{2}\right)^{2}} \frac{1}{J\left(k_{2}\right)\left(m^{2}-\left(k_{2}+p_{12}\right)^{2}\right)\left(m^{2}-\left(k_{2}-p_{3}\right)^{2}\right)},
$$


where the Jacobian of the contour deformation $J\left(k_{2}\right)$ reads,

$$
J\left(k_{2}\right)=s_{12}^{2} \sqrt{s_{12}\left(s_{12}\left(2 p_{1} \cdot k_{2}+k_{2}^{2}+m^{2}\right)^{2}-4 m^{2}\left(k_{2}^{2} s_{12}-4 p_{1} \cdot k_{2} p_{2} \cdot k_{2}\right)\right)} .
$$

We note that in the limit $m^{2} \rightarrow 0$ the Jacobian reduces to,

$$
\left.J\left(k_{2}\right)\right|_{m^{2} \rightarrow 0}=s_{12}^{3}\left(k_{2}+p_{1}\right)^{2},
$$

reproducing the well known result for the cut of the massless case.

Localizing the contour onto the two genuine propagators of (F.4), will yield an expression similar to (F.2) - an inverse square root of a quartic polynomial with no repeated roots.

Open Access. This article is distributed under the terms of the Creative Commons Attribution License (CC-BY 4.0), which permits any use, distribution and reproduction in any medium, provided the original author(s) and source are credited.

\section{References}

[1] D. Graudenz, M. Spira and P.M. Zerwas, QCD corrections to Higgs boson production at proton proton colliders, Phys. Rev. Lett. 70 (1993) 1372 [INSPIRE].

[2] M. Spira, A. Djouadi, D. Graudenz and P.M. Zerwas, Higgs boson production at the LHC, Nucl. Phys. B 453 (1995) 17 [hep-ph/9504378] [INSPIRE].

[3] R.K. Ellis, I. Hinchliffe, M. Soldate and J.J. van der Bij, Higgs Decay to $\tau^{+} \tau^{-}$: A Possible Signature of Intermediate Mass Higgs Bosons at the SSC, Nucl. Phys. B 297 (1988) 221 [INSPIRE].

[4] R.P. Kauffman, Higgs boson $p_{T}$ in gluon fusion, Phys. Rev. D 44 (1991) 1415 [INSPIRE].

[5] M. Grazzini and H. Sargsyan, Heavy-quark mass effects in Higgs boson production at the LHC, JHEP 09 (2013) 129 [arXiv:1306.4581] [INSPIRE].

[6] C. Anastasiou, C. Duhr, F. Dulat, F. Herzog and B. Mistlberger, Higgs Boson Gluon-Fusion Production in QCD at Three Loops, Phys. Rev. Lett. 114 (2015) 212001 [arXiv:1503.06056] [INSPIRE].

[7] C. Anastasiou et al., High precision determination of the gluon fusion Higgs boson cross-section at the LHC, JHEP 05 (2016) 058 [arXiv: 1602.00695] [INSPIRE].

[8] U. Baur and E.W.N. Glover, Higgs Boson Production at Large Transverse Momentum in Hadronic Collisions, Nucl. Phys. B 339 (1990) 38 [InSPIRE].

[9] D. de Florian, M. Grazzini and Z. Kunszt, Higgs production with large transverse momentum in hadronic collisions at next-to-leading order, Phys. Rev. Lett. 82 (1999) 5209 [hep-ph/9902483] [INSPIRE].

[10] R. Boughezal, F. Caola, K. Melnikov, F. Petriello and M. Schulze, Higgs boson production in association with a jet at next-to-next-to-leading order, Phys. Rev. Lett. 115 (2015) 082003 [arXiv: 1504.07922] [INSPIRE].

[11] R. Boughezal, C. Focke, W. Giele, X. Liu and F. Petriello, Higgs boson production in association with a jet at NNLO using jettiness subtraction, Phys. Lett. B 748 (2015) 5 [arXiv: 1505. 03893] [INSPIRE]. 
[12] X. Chen, J. Cruz-Martinez, T. Gehrmann, E.W.N. Glover and M. Jaquier, NNLO QCD corrections to Higgs boson production at large transverse momentum, JHEP 10 (2016) 066 [arXiv: 1607.08817] [INSPIRE].

[13] R.V. Harlander, T. Neumann, K.J. Ozeren and M. Wiesemann, Top-mass effects in differential Higgs production through gluon fusion at order $\alpha_{s}^{4}$, JHEP 08 (2012) 139 [arXiv:1206.0157] [INSPIRE].

[14] R. Frederix, S. Frixione, E. Vryonidou and M. Wiesemann, Heavy-quark mass effects in Higgs plus jets production, JHEP 08 (2016) 006 [arXiv: 1604.03017] [INSPIRE].

[15] F. Caola, S. Forte, S. Marzani, C. Muselli and G. Vita, The Higgs transverse momentum spectrum with finite quark masses beyond leading order, JHEP 08 (2016) 150 [arXiv: 1606.04100] [INSPIRE].

[16] T. Neumann and C. Williams, The Higgs boson at high $p_{T}$, arXiv:1609.00367 [INSPIRE].

[17] C. Grojean, E. Salvioni, M. Schlaffer and A. Weiler, Very boosted Higgs in gluon fusion, JHEP 05 (2014) 022 [arXiv: 1312.3317] [INSPIRE].

[18] A. Azatov and A. Paul, Probing Higgs couplings with high $p_{T}$ Higgs production, JHEP 01 (2014) 014 [arXiv:1309.5273] [INSPIRE].

[19] A. Azatov, C. Grojean, A. Paul and E. Salvioni, Resolving gluon fusion loops at current and future hadron colliders, JHEP 09 (2016) 123 [arXiv: 1608.00977] [INSPIRE].

[20] A.V. Kotikov, Differential equations method: New technique for massive Feynman diagrams calculation, Phys. Lett. B 254 (1991) 158 [INSPIRE].

[21] A.V. Kotikov, Differential equation method: The Calculation of $N$ point Feynman diagrams, Phys. Lett. B 267 (1991) 123 [Erratum ibid. B 295 (1992) 409] [INSPIRE].

[22] Z. Bern, L.J. Dixon and D.A. Kosower, Dimensionally regulated pentagon integrals, Nucl. Phys. B 412 (1994) 751 [hep-ph/9306240] [INSPIRE].

[23] E. Remiddi, Differential equations for Feynman graph amplitudes, Nuovo Cim. A 110 (1997) 1435 [hep-th/9711188] [INSPIRE].

[24] T. Gehrmann and E. Remiddi, Differential equations for two loop four point functions, Nucl. Phys. B 580 (2000) 485 [hep-ph/9912329] [INSPIRE].

[25] F.V. Tkachov, A Theorem on Analytical Calculability of Four Loop Renormalization Group Functions, Phys. Lett. B 100 (1981) 65 [INSPIRE].

[26] K.G. Chetyrkin and F.V. Tkachov, Integration by Parts: The Algorithm to Calculate -functions in 4 Loops, Nucl. Phys. B 192 (1981) 159 [INSPIRE].

[27] S. Laporta and E. Remiddi, The analytical value of the electron $(g-2)$ at order $\alpha^{3}$ in QED, Phys. Lett. B 379 (1996) 283 [hep-ph/9602417] [INSPIRE].

[28] S. Laporta, High precision calculation of multiloop Feynman integrals by difference equations, Int. J. Mod. Phys. A 15 (2000) 5087 [hep-ph/0102033] [INSPIRE].

[29] A.B. Goncharov, Multiple polylogarithms, cyclotomy and modular complexes, Math. Res. Lett. 5 (1998) 497 [arXiv:1105. 2076] [INSPIRE].

[30] E. Remiddi and J.A.M. Vermaseren, Harmonic polylogarithms, Int. J. Mod. Phys. A 15 (2000) 725 [hep-ph/9905237] [INSPIRE]. 
[31] A.B. Goncharov, M. Spradlin, C. Vergu and A. Volovich, Classical Polylogarithms for Amplitudes and Wilson Loops, Phys. Rev. Lett. 105 (2010) 151605 [arXiv:1006.5703] [INSPIRE].

[32] C. Duhr, H. Gangl and J.R. Rhodes, From polygons and symbols to polylogarithmic functions, JHEP 10 (2012) 075 [arXiv:1110.0458] [INSPIRE].

[33] H. Frellesvig, D. Tommasini and C. Wever, On the reduction of generalized polylogarithms to $L i_{n}$ and $L i_{2,2}$ and on the evaluation thereof, JHEP 03 (2016) 189 [arXiv:1601.02649] [INSPIRE].

[34] J.M. Henn, Multiloop integrals in dimensional regularization made simple, Phys. Rev. Lett. 110 (2013) 251601 [arXiv:1304.1806] [INSPIRE].

[35] F. Cachazo, Sharpening The Leading Singularity, arXiv:0803.1988 [INSPIRE].

[36] N. Arkani-Hamed, J.L. Bourjaily, F. Cachazo and J. Trnka, Local Integrals for Planar Scattering Amplitudes, JHEP 06 (2012) 125 [arXiv: 1012.6032] [INSPIRE].

[37] J.M. Henn, Modern methods for scattering amplitudes, lectures given at Nordita School on Integrability, Nordita, Stockholm, 4-12 August 2014, http://www.nordita.org/video/index.php?ev=integrability2014.

[38] J.M. Henn, Lectures on differential equations for Feynman integrals, J. Phys. A 48 (2015) 153001 [arXiv:1412.2296] [InSPIRE].

[39] R.N. Lee, Reducing differential equations for multiloop master integrals, JHEP 04 (2015) 108 [arXiv: 1411.0911] [INSPIRE].

[40] J.M. Henn and V.A. Smirnov, Analytic results for two-loop master integrals for Bhabha scattering I, JHEP 11 (2013) 041 [arXiv:1307.4083] [INSPIRE].

[41] J.M. Henn, A.V. Smirnov and V.A. Smirnov, Evaluating single-scale and/or non-planar diagrams by differential equations, JHEP 03 (2014) 088 [arXiv:1312.2588] [INSPIRE].

[42] M. Argeri et al., Magnus and Dyson Series for Master Integrals, JHEP 03 (2014) 082 [arXiv: 1401.2979] [INSPIRE].

[43] J.M. Henn, K. Melnikov and V.A. Smirnov, Two-loop planar master integrals for the production of off-shell vector bosons in hadron collisions, JHEP 05 (2014) 090 [arXiv: 1402.7078] [INSPIRE].

[44] T. Gehrmann, A. von Manteuffel, L. Tancredi and E. Weihs, The two-loop master integrals for $q \bar{q} \rightarrow V V$, JHEP 06 (2014) 032 [arXiv: 1404.4853] [INSPIRE].

[45] F. Caola, J.M. Henn, K. Melnikov and V.A. Smirnov, Non-planar master integrals for the production of two off-shell vector bosons in collisions of massless partons, JHEP 09 (2014) 043 [arXiv: 1404.5590] [INSPIRE].

[46] S. Di Vita, P. Mastrolia, U. Schubert and V. Yundin, Three-loop master integrals for ladder-box diagrams with one massive leg, JHEP 09 (2014) 148 [arXiv:1408.3107] [INSPIRE].

[47] G. Bell and T. Huber, Master integrals for the two-loop penguin contribution in non-leptonic B-decays, JHEP 12 (2014) 129 [arXiv: 1410. 2804] [INSPIRE].

[48] R. Bonciani, V. Del Duca, H. Frellesvig, J.M. Henn, F. Moriello and V.A. Smirnov, Next-to-leading order QCD corrections to the decay width $H \rightarrow Z \gamma$, JHEP 08 (2015) 108 [arXiv: 1505.00567] [INSPIRE]. 
[49] T. Gehrmann, S. Guns and D. Kara, The rare decay $H \rightarrow Z \gamma$ in perturbative $Q C D$, JHEP 09 (2015) 038 [arXiv: 1505.00561] [INSPIRE].

[50] A. Grozin, J.M. Henn, G.P. Korchemsky and P. Marquard, The three-loop cusp anomalous dimension in QCD and its supersymmetric extensions, JHEP 01 (2016) 140 [arXiv: 1510.07803] [INSPIRE].

[51] T. Gehrmann, J.M. Henn and N.A. Lo Presti, Analytic form of the two-loop planar five-gluon all-plus-helicity amplitude in QCD, Phys. Rev. Lett. 116 (2016) 062001 [Erratum ibid. 116 (2016) 189903] [arXiv:1511.05409] [INSPIRE].

[52] J.M. Henn, A.V. Smirnov, V.A. Smirnov and M. Steinhauser, A planar four-loop form factor and cusp anomalous dimension in QCD, JHEP 05 (2016) 066 [arXiv: 1604.03126] [INSPIRE].

[53] R. Bonciani, S. Di Vita, P. Mastrolia and U. Schubert, Two-Loop Master Integrals for the mixed EW-QCD virtual corrections to Drell-Yan scattering, JHEP 09 (2016) 091 [arXiv: 1604.08581] [INSPIRE].

[54] J.M. Henn and B. Mistlberger, Four-Gluon Scattering at Three Loops, Infrared Structure and the Regge Limit, Phys. Rev. Lett. 117 (2016) 171601 [arXiv:1608.00850] [INSPIRE].

[55] R.N. Lee and V.A. Smirnov, Evaluating the last missing ingredient for the three-loop quark static potential by differential equations, JHEP 10 (2016) 089 [arXiv:1608.02605] [INSPIRE].

[56] K.-T. Chen, Iterated path integrals, Bull. Am. Math. Soc. 83 (1977) 831 [InSPIRE].

[57] S. Caron-Huot and J.M. Henn, Iterative structure of finite loop integrals, JHEP 06 (2014) 114 [arXiv:1404.2922] [INSPIRE].

[58] L. Tancredi, Integration by parts identities in integer numbers of dimensions. A criterion for decoupling systems of differential equations, Nucl. Phys. B 901 (2015) 282 [arXiv: 1509.03330] [INSPIRE].

[59] S. Caron-Huot and K.J. Larsen, Uniqueness of two-loop master contours, JHEP 10 (2012) 026 [arXiv:1205.0801] [INSPIRE].

[60] M. Caffo, H. Czyz, S. Laporta and E. Remiddi, The Master differential equations for the two loop sunrise selfmass amplitudes, Nuovo Cim. A 111 (1998) 365 [hep-th/9805118] [INSPIRE].

[61] S. Laporta and E. Remiddi, Analytic treatment of the two loop equal mass sunrise graph, Nucl. Phys. B 704 (2005) 349 [hep-ph/0406160] [INSPIRE].

[62] S. Bloch and P. Vanhove, The elliptic dilogarithm for the sunset graph, J. Number Theor. 148 (2015) 328 [arXiv:1309.5865] [INSPIRE].

[63] S. Bloch, M. Kerr and P. Vanhove, A Feynman integral via higher normal functions, Compos. Math. 151 (2015) 2329 [arXiv:1406.2664] [INSPIRE].

[64] L. Adams, C. Bogner and S. Weinzierl, The two-loop sunrise graph in two space-time dimensions with arbitrary masses in terms of elliptic dilogarithms, J. Math. Phys. 55 (2014) 102301 [arXiv:1405.5640] [INSPIRE].

[65] L. Adams, C. Bogner and S. Weinzierl, The two-loop sunrise integral around four space-time dimensions and generalisations of the Clausen and Glaisher functions towards the elliptic case, J. Math. Phys. 56 (2015) 072303 [arXiv:1504.03255] [INSPIRE]. 
[66] L. Adams, C. Bogner and S. Weinzierl, The iterated structure of the all-order result for the two-loop sunrise integral, J. Math. Phys. 57 (2016) 032304 [arXiv:1512.05630] [INSPIRE].

[67] S. Bloch, M. Kerr and P. Vanhove, Local mirror symmetry and the sunset Feynman integral, arXiv: 1601.08181 [INSPIRE].

[68] E. Remiddi and L. Tancredi, Differential equations and dispersion relations for Feynman amplitudes. The two-loop massive sunrise and the kite integral, Nucl. Phys. B 907 (2016) 400 [arXiv:1602.01481] [inSPIRE].

[69] L. Adams, C. Bogner, A. Schweitzer and S. Weinzierl, The kite integral to all orders in terms of elliptic polylogarithms, arXiv:1607.01571 [INSPIRE].

[70] C.G. Papadopoulos, Simplified differential equations approach for Master Integrals, JHEP 07 (2014) 088 [arXiv: 1401.6057] [INSPIRE].

[71] A.V. Smirnov, Algorithm FIRE - Feynman Integral REduction, JHEP 10 (2008) 107 [arXiv:0807.3243] [INSPIRE].

[72] A.V. Smirnov and V.A. Smirnov, FIRE4, LiteRed and accompanying tools to solve integration by parts relations, Comput. Phys. Commun. 184 (2013) 2820 [arXiv:1302.5885] [INSPIRE].

[73] A.V. Smirnov, FIRE5: a C++ implementation of Feynman Integral REduction, Comput. Phys. Commun. 189 (2015) 182 [arXiv:1408.2372] [INSPIRE].

[74] R.N. Lee, Presenting LiteRed: a tool for the Loop InTEgrals REDuction, arXiv:1212.2685 [INSPIRE].

[75] F.C.S. Brown, Multiple zeta values and periods of moduli spaces $\mathfrak{M}_{0, n}$, Annales Sci. Ecole Norm. Sup. 42 (2009) 371 [math/0606419] [INSPIRE].

[76] V.A. Smirnov, Asymptotic expansions in limits of large momenta and masses, Commun. Math. Phys. 134 (1990) 109 [InSPIRE].

[77] V.A. Smirnov, Asymptotic expansions in momenta and masses and calculation of Feynman diagrams, Mod. Phys. Lett. A 10 (1995) 1485 [hep-th/9412063] [INSPIRE].

[78] V.A. Smirnov, Applied asymptotic expansions in momenta and masses, Springer Tracts Mod. Phys. 177 (2002) 1 [INSPIRE].

[79] R.K. Ellis and G. Zanderighi, Scalar one-loop integrals for QCD, JHEP 02 (2008) 002 [arXiv:0712.1851] [INSPIRE].

[80] A.V. Smirnov and M.N. Tentyukov, Feynman Integral Evaluation by a Sector decomposiTion Approach (FIESTA), Comput. Phys. Commun. 180 (2009) 735 [arXiv:0807.4129] [INSPIRE].

[81] A.V. Smirnov, V.A. Smirnov and M. Tentyukov, FIESTA 2: Parallelizeable multiloop numerical calculations, Comput. Phys. Commun. 182 (2011) 790 [arXiv:0912.0158] [INSPIRE].

[82] A.V. Smirnov, FIESTA 3: cluster-parallelizable multiloop numerical calculations in physical regions, Comput. Phys. Commun. 185 (2014) 2090 [arXiv: 1312.3186] [inSPIRE].

[83] B.T. Whittaker and G.N. Watson, A Course of Modern Analysis, 4th edition, Cambridge University Press, London (1958), pg. 206-208.

[84] L. Tancredi, Differential equations and dispersion relations for Feynman amplitudes, talk given at LoopFest 2016, https://indico.fnal.gov/event/loopfestXV. 
[85] J. Broedel, C.R. Mafra, N. Matthes and O. Schlotterer, Elliptic multiple zeta values and one-loop superstring amplitudes, JHEP 07 (2015) 112 [arXiv:1412.5535] [INSPIRE].

[86] F.C.S. Brown and A. Levin, Multiple Elliptic Polylogarithms, arXiv:1110.6917.

[87] J. Kuipers, T. Ueda, J.A.M. Vermaseren and J. Vollinga, FORM version 4.0, Comput. Phys. Commun. 184 (2013) 1453 [arXiv:1203.6543] [InSPIRE].

[88] J.A.M. Vermaseren, Axodraw, Comput. Phys. Commun. 83 (1994) 45 [inSPIRE].

[89] D.A. Kosower and K.J. Larsen, Maximal Unitarity at Two Loops, Phys. Rev. D 85 (2012) 045017 [arXiv:1108.1180] [InSPIRE].

[90] L.J. Dixon, Calculating scattering amplitudes efficiently, hep-ph/9601359 [INSPIRE].

[91] J.M. Drummond, J. Henn, G.P. Korchemsky and E. Sokatchev, Dual superconformal symmetry of scattering amplitudes in $N=4$ super-Yang-Mills theory, Nucl. Phys. B 828 (2010) 317 [arXiv:0807.1095] [InSPIRE].

[92] A. Hodges, The Box Integrals in Momentum-Twistor Geometry, JHEP 08 (2013) 051 [arXiv: 1004.3323] [INSPIRE].

[93] M. Søgaard and Y. Zhang, Elliptic Functions and Maximal Unitarity, Phys. Rev. D 91 (2015) 081701 [arXiv:1412.5577] [INSPIRE]. 